Article

\title{
Species Composition and New Records of Diatom Taxa on Phyllodictyon pulcherrimum (Chlorophyceae) from the Gulf of California
}

\author{
Francisco Omar López-Fuerte ${ }^{1}\left(\mathbb{D}\right.$, David Alfaro Siqueiros Beltrones ${ }^{2, *}$ (i) \\ and María del Carmen Altamirano-Cerecedo ${ }^{3}$ \\ 1 Departamento Académico de Ciencias Marinas y Costeras, Universidad Autónoma de Baja California Sur, \\ Carretera al Sur, km. 5.5, La Paz C.P. 23080, Baja California Sur, Mexico; folopez@uabcs.mx \\ 2 Departamento Plancton y Ecología Marina, Instituto Politécnico Nacional, \\ Centro Interdisciplinario de Ciencias Marinas, Av. Instituto Politécnico Nacional S/N, \\ Col. Playa Palo de Santa Rita, La Paz C.P. 23096, Baja California Sur, Mexico \\ 3 Departamento Académico de Economía, Universidad Autónoma de Baja California Sur, Carretera al Sur, \\ km. 5.5, La Paz C.P. 23080, Baja California Sur, Mexico; malt@uabcs.mx \\ * Correspondence: dsiquei@gmail.com
}

Received: 18 July 2020; Accepted: 2 September 2020; Published: 4 September 2020

\begin{abstract}
A taxonomic analysis of diatoms found on Phyllodictyon pulcherrimum yielded a total of 244 diatom taxa (all illustrated) within 27 orders, 45 families, and 86 genera. The Taxa were briefly documented in a list including identification references and morphometric data. Thirty-eight of the taxa identified at the species and infraspecific levels represent new records for the coasts of Mexico. Seven were recorded for the first time on the American continent: Auricula flabelliformis, A. pulchra, Campylodiscus scalaris, Coscinodiscus mesoleius, Dimeregramma fulvum, Navicula palpebralis var. angulosa, and Seminavis barbarae, and one, Nitzschia fusiformis, for the Pacific Ocean. This is the second record of the chlorophyte P. pulcherrimum in the north Pacific and the third for Mexican waters. The results confirm that surveying rare macroalgae species as hosts for epiphytic diatoms provides opportunities to seek new records of diatom taxa, or even new taxa, in regions around the world.
\end{abstract}

Keywords: Bacillariophyta; epiphytes; floristics; Gulf of California; new records

\section{Introduction}

Benthic diatoms thrive on a great variety of substrates that include both live and inert surfaces [1-3]. Among the former, the surfaces of macroalgae provide, by far, the highest species richness of epiphytic diatoms that may number several hundred species [4]. However, many macroalgae taxa worldwide have yet to be surveyed for epiphytic diatom floristics. The few studies of diatom epiphytes on macroalgae cover hosts of the three main groups: brown, red, and green [5-10]. Specific studies on epiphytic diatoms of green macroalgae that include Caulerpa taxifolia, C. verticillata, Chaetomorpha linum, Cladophora glomerata, Cladophora sp., Halimeda tuna, H. sp., Ulva intestinalis, and U. lactuca have recorded a total of 300 taxa $[5,10-20]$.

For the Mexican region, the only studies are those by $[6,21]$ that recorded 89 diatom taxa on Codium spp. and 51 on Codium latum subsp. palmeri. Most research on epiphytic diatoms of macroalgae in Mexico has been conducted in the NW region [22], while for the coasts of the Gulf of Mexico only one such study has been published. It deals with rhodophytes, yielding 115 epiphytic diatom taxa from specimens of six rhodophyte species that included six new records for Mexican waters [8]. In contrast, 208 (specific and infraspecific) epiphytic diatom taxa were identified in samples from two specimens of Laurencia collected off the Revillagigedo Islands, 16 of which could not be identified at the species level 
and so may represent new taxa. Another 52 were new additions to the diatom flora of Mexico's coasts, and three to Mexico's Pacific coast [22].

Considering the numerous macrophyte taxa found on the coasts of the California-Baja California Peninsula, it is clear that studies of epiphytic diatoms in the eastern Pacific are lacking, including works on well-known macroalgae. For example, the absence of the diatom Pteroncola inane in other floristic lists in NW Mexico, and a recently-observed high abundance on Eisenia arborea, suggest that this host may have specific conditions that favor the growth of this and other new records [23]. Finally, at least 200 epiphytic diatom taxa have been recorded on various macroalgae species in the Gulf of California [6].

In addition, surveying rare macroalgae species as hosts for epiphytic diatoms offers the opportunity to seek new records of diatom taxa, or even new taxa. Recently, a specimen that constitutes the second record of the chlorophyte Phyllodictyon pulcherrimum in the North Pacific (third for Mexican coasts) was collected off the west coast of the Gulf of California, prompting our interest in the species composition of its epiphytic diatom flora. A fresh inspection of the macroalgal specimen surface showed an epiphytic diatom flora apparently composed exclusively of Cocconeis species. However, because epiphytic diatom taxocoenoses are likely the richest in taxonomic diversity, our objective was to identify the diatom taxa found on the specimen of P. pulcherrimum collected in the central zone of the west coast of the Baja California Peninsula and describe its potential floristics.

\section{Materials and Methods}

In July 2019, a single thallus of a green seaweed was collected from the west coast of the central region of the Gulf of California, $4 \mathrm{~km}$ south of Santa Rosalia (Baja California Sur, Mexico) at $27^{\circ} 19^{\prime} 66^{\prime \prime} \mathrm{N}, 112^{\circ} 14^{\prime} 00.13^{\prime \prime} \mathrm{W}$ (Figure 1). The sampling site is characterized by rocky substrata and was occupied by macroalgae of various genera (e.g., Botryocladia, Dictyota, Padina) and fringed by sandy substrata harboring young colonies of black coral Antipatharia sp. The green algae specimen was retrieved manually at a depth of $18 \mathrm{~m}$ using semiautonomous diving equipment (hookah), and later identified as P. pulcherrimum, based on morphological characteristics following [24,25]. The specimen was then housed in the Phycological Herbarium of the Universidad Autónoma de Baja California Sur [FBCS-20172].

During the process of identifying P. pulcherrimum, a $3 \mathrm{~cm}^{2}$ segment of the thallus was observed under a microscope to describe the cell morphology. This revealed a layer of diatoms, mainly Cocconeis-like forms (Figure 2). The segment of algae was processed to separate and clean the diatoms using nitric acid and commercial ethanol (90\%) in a test tube at a 2:2 ratio, applying heat with a burner for around $30 \mathrm{~s}$. The oxidized material was transferred to a $15-\mathrm{mL}$ Falcon tube, diluted with deionized water, and centrifuged for $5 \mathrm{~min}$ at $4000 \times g \mathrm{rpm}$. This was repeated five times until reaching a circumneutral $\mathrm{pH}$. Clean aliquots of the sample were mounted on permanent slides using Zyrax ${ }^{\circledR}$ $(\mathrm{RI}=1.7)$ (made and distributed by Bill Daily, University of Pennsylvania). Six mounted slides were observed under a Zeiss ${ }^{\circledR}$ Axio Lab A1 (Zeiss, Germany) compound microscope equipped with phase contrast and a Canon 5D mark II camera (Canon, Japan). Identification of each taxon was carried out using the reference that appears in the corresponding description.

A formal list of the diatom taxa was constructed following [26], including information on references and morphometrics. Nomenclatural updates were based on AlgaeBase [27] and www.marinespecies.org [28], complemented by an iconographic catalog of all the taxa registered.

To contrast our observations, 14 publications on epiphytic diatom floristics for various green seaweed taxa were reviewed. These included Caulerpa taxifolia, C. verticillata, Chaetomorpha linum, Cladophora glomerata, Cladophora sp., Codium latum subsp. palmeri, Codium spp., Halimeda tuna, Halimeda sp., Ulva intestinalis, and U. lactuca $[5,6,9,11-21]$. 


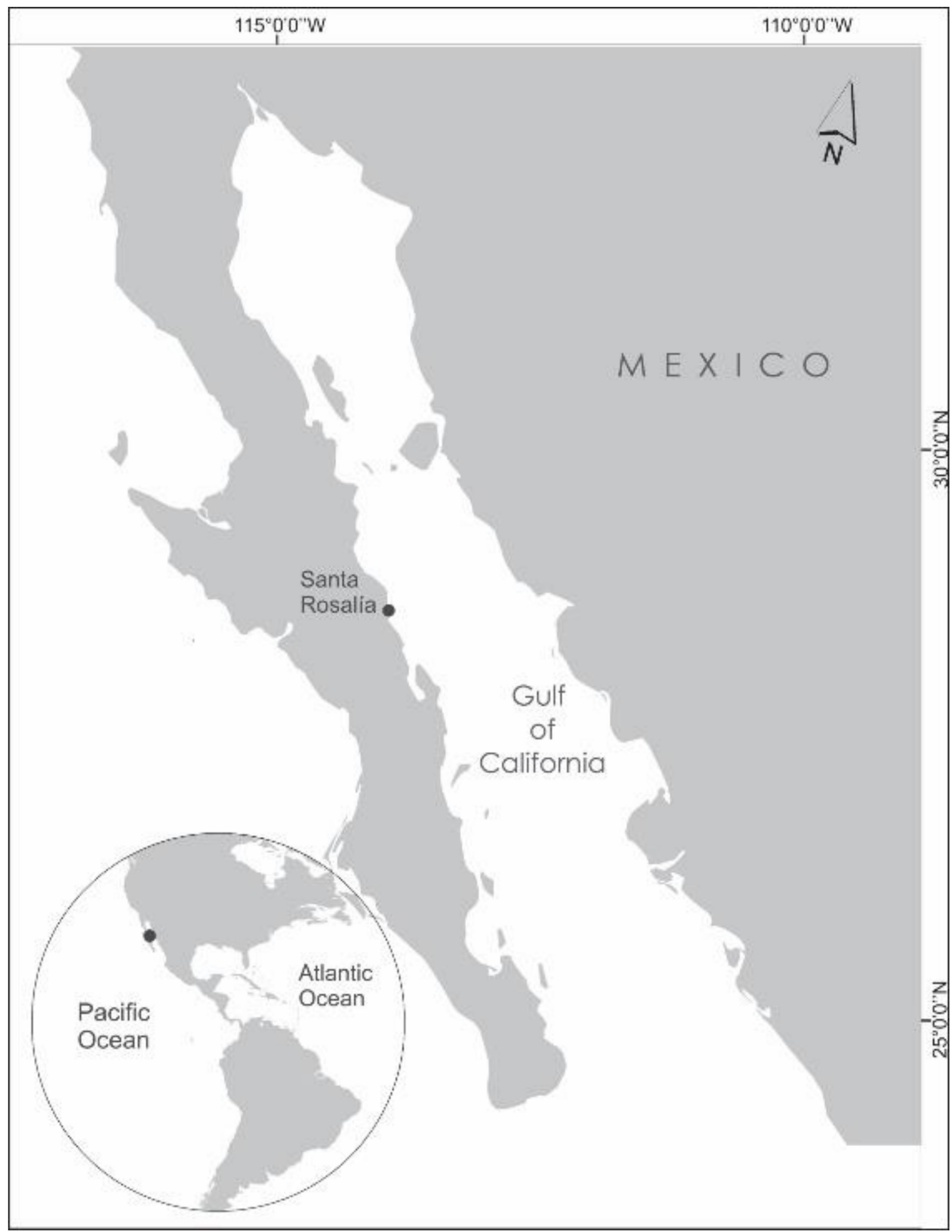

Figure 1. Location of the sampling site off Santa Rosalia, Baja California Sur, Mexico. 


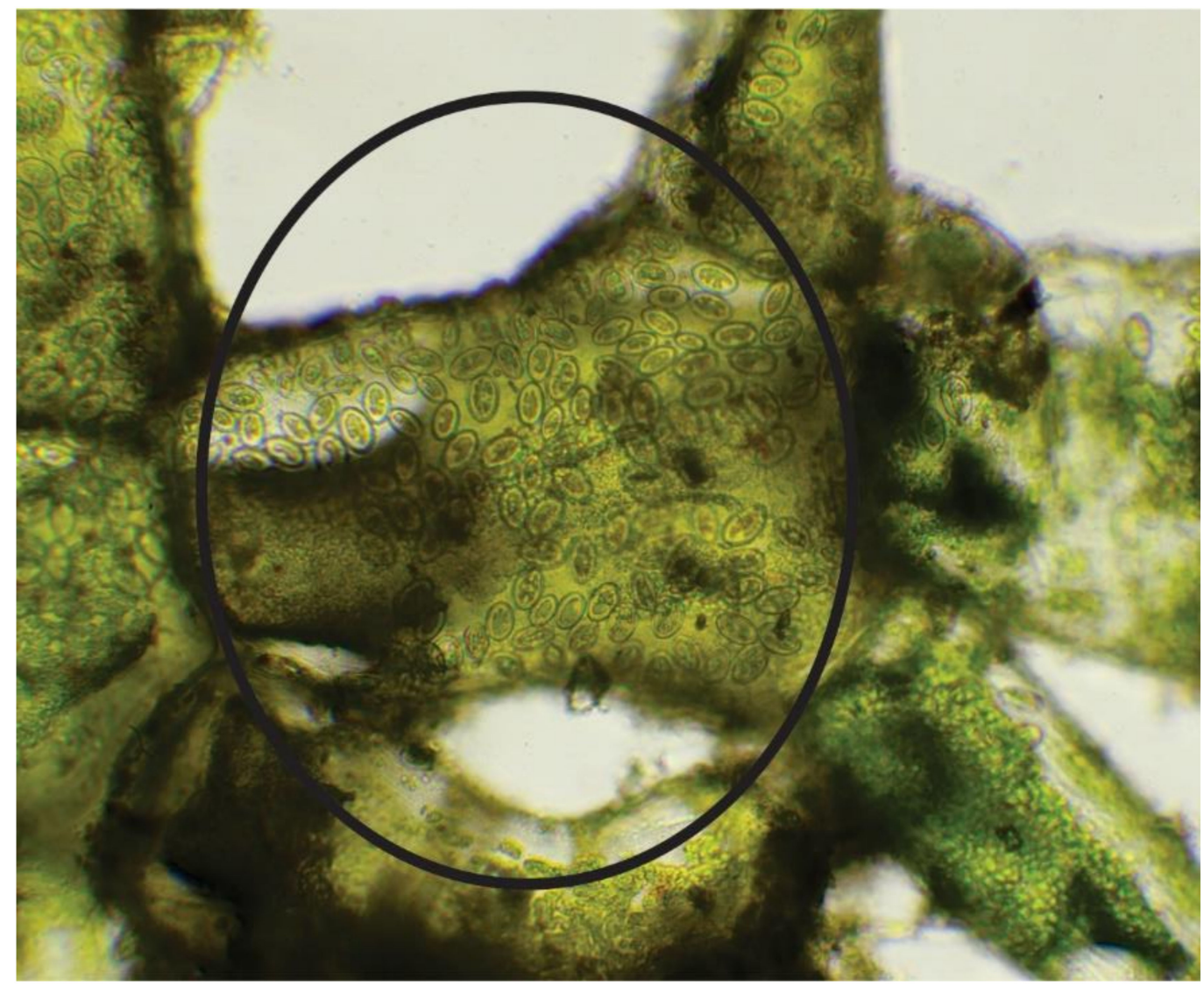

Figure 2. Segment of the thallus of Phyllodictyon pulcherrimum exhibiting a layer of diatoms, mainly Cocconeis spp.

\section{Results}

A thorough scanning of the six mounted slides yielded 244 specific and infraspecific taxa, belonging to 27 orders, 45 families, and 86 genera. Only three taxa were solely identified at the genus level, and five as cf. All the diatom taxa found on P. pulcherrimum are displayed in an iconographic catalogue (Figures 3-46) with 615 images that show distinct focal planes, sizes and views (valve and girdle). The genera best represented were Nitzschia (25 taxa), Amphora (17), Diploneis (15), Navicula (13), and Cocconeis (12), as they accounted for $34 \%$ of all taxa. In contrast, for 49 genera only a single taxon was recorded, representing $57 \%$ of all the records collected. Overall, 38 taxa $(15.5 \%)$ are new records for Mexico's coasts.

At the class level, 68\% (25 taxa) of the new records are Bacillariophyceae, $21 \%$ (8) are Coscinodiscophyceae, and 11\% (4) are Fragilariophyceae. These were included in 26 genera, of which 19 (73\%) were represented by a single taxon. Nitzschia and Gyrosigma had the most taxa-five and three, respectively-while six others had two taxa each (Auricula, Diploneis, Navicula, Parlibellus, Pleurosigma, and Synedra).

Following is a systematic list of epiphytic diatoms (Bacillariophyta) of Phyllodictyon pulcherrimum from the Gulf of California. * New record for Mexican coasts. ${ }^{+}$New record for the American Continent. $n=$ number of specimens measured. Slides containing the diatom taxa recorded are refered to the macroalgal specimen housed at the Phycological Herbarium of the Universidad Autónoma de Baja California Sur [FBCS-20172].

CLASS: COSCINODISCOPHYCEAE F.E. Round and R.M. Crawford 1990

Order: Asterolamprales F.E. Round and R.M. Crawford 1990

Family: Asterolampraceae H.L. Smith 1872 
ASTEROMPHALUS C.G. Ehrenberg 1844

Asteromphalus arachne (A. Brébisson) J. Ralfs 1861

Reference illustrate (Ref. illus.): Pritchard, A. 1861, p. 837, pl. 5, Figure 66; Hasle, G.R., Syvertsen, E.E. 1996, p. 137, pl. 25.

Diameter $45 \mu \mathrm{m}$, areolae 7 in $10 \mu \mathrm{m}(n=1)$ (Figure 10c).

Order: Biddulphiales H. Krieger 1954

Family: Biddulphiaceae F.T. Kützing 1844

BIDDULPHIA S.F. Gray 1821

Biddulphia biddulphiana (J.E. Smith) C.S. Boyer 1900

Ref. illus.: Hustedt, F. 1930, p. 832, Figure 490; Witkowski, A.; Lange-Bertalot, H.; Metzeltin, D. 2000, p. 25, pl. 8, Figures 8 and 9 (as B. pulchella).

Size: 40-65 $\mu \mathrm{m}$ long, 33-102 $\mu \mathrm{m}$ broad, pervalvar axis 85-131 $\mu \mathrm{m}$, areolae on the mantle 4 to 5 in $10 \mu \mathrm{m}$ $(n=7)$ (Figure 12c-i).

Remark: Valves elliptic, swollen margin, strongly sculptured, divided into three sections by strong costae. Ends of the valve furnished with large globular process covered with fine pores, areolae arranged in longitudinal and transverse rows, girdle punctate in longitudinal lines.

\section{Biddulphia tridentata C.G. Ehrenberg 1844 *}

Ref. illus.: Schmidt, A.W.F. 1888, pl. 118, Figures 13-18.

Size: $90 \mu \mathrm{m}$ long, $58 \mu \mathrm{m}$ broad, areolae 5-6 in $10 \mu \mathrm{m}(n=1)$ (Figure 11d,e).

Remark: Rimoportula in B. biddulphiana are located toward the center of the valve, while in $B$. tridentata they are displaced to the margins. Likewise, costae in the latter show a hyaline area but no B. biddulphiana.

\section{Biddulphia tridens (C.G. Ehrenberg) C.G. Ehrenberg 1841}

Ref. illus.: Roper, F.C.S. 1859, p. 8, pl. 1, Figures 1 and 2; Foged, N. 1975, p. 15, pl. 1, Figures 6 and 7. Size: $46-106 \mu \mathrm{m}$ long, $31 \mu \mathrm{m}$ broad, areolae 6 to 7 in $10 \mu \mathrm{m}(n=3)$ (Figure 11a-c,f).

Remark: Figure 11a,b show a clear difference in size and form of the pseudo-ocelii, but said variations seem insufficient to render the specimens a distinct species from B. tridens. Although in [29] pl. 119, the specimens from Figures 15 and 17 with short-flat pseudo-ocelii (Figure 11a,b) are considered a variety of B. tridens, in [30] said variation may be present individually, e.g., Figure 3, which shows classic long-rounded pseudo-ocelii, while Figure 44 (hipovalve) shows short-flat pseudo-ocelii.

LAMPRISCUS A.W.F. Schmidt 1882

\section{Lampriscus shadboltianum (R.K. Greville) H. Peragallo and M. Peragallo 1902}

Ref. illus.: Peragallo, H.; Peragallo, M. 1897-1908, p. 389, pl. 106, Figure 1; Navarro, J.N. 1981, p. 618, Figures 33-36; Vidal, L.A.; Ospino-Acosta, K.; Linares-Vargas, K.; García-Urueña, R. 2017, p. 56, Figures $5 \mathrm{~g}$ and $6 \mathrm{a}$.

Diameter 58-73 $\mu \mathrm{m}$, pervalvar axis $100 \mu \mathrm{m}$, areolae 6-8 in $10 \mu \mathrm{m}(n=6)$ (Figure 14a-g).

Order: Chaetocerotales F.E. Round and R.M. Crawford 1990

Family Chaetocerotaceae J. Ralfs 1861

BACTERIASTRUM G. Shadbolt 1854

Bacteriastrum hyalinum H.S. Lauder 1864

Ref. illus.: Lauder, H.S. 1864, p. 8, pl. 3, Figure 7a,b; Bosak, S.; Šupraha, L.; Nanjappa, D.; Kooistra, W.H.C.F.; Sarno, D. 2015, p. 135, Figures 18-36.

Diameter $13 \mu \mathrm{m}(n=2)$ (Figure 10a,b).

Order: Eupodiscales V.A. Nikolaev and D.M. Harwood 2000

Family: Odontellaceae P.A. Sims, D.M. Williams and M.P. Ashworth 2018 
ODONTELLA C.A. Agardh 1832

Odontella obtusa F.T. Kützing 1844

Ref. illus.: Kützing, F.T. 1844, p. 137, pl. 18/8, Figures 1-3, 6-8.

Size: $100-127 \mu \mathrm{m}$ long, $65-92 \mu \mathrm{m}$ broad, 5 areolae in $10 \mu \mathrm{m}(n=4)$ (Figure 13a-d).

Odontella rostrata (F. Hustedt) R. Simonsen 1987

Ref. illus.: Hustedt, F. 1939, p. 591, Figures 5-7; Simonsen, R. 1987, p. 250, pl. 373, Figures 1-11 (both as Biddulphia rostrata); Sims, P.A.; Williams, D.M.; Ashworth, M., p. 32, Figures 11-116.

Size: $19 \mu \mathrm{m}$ long, $8-11$ areolae in $10 \mu \mathrm{m}(n=2)$ (Figure 12a,b).

Remark: According to [31], O. aurita differs from O. rostrata by having convex valves with elevated central areas, a valve mantle turned towards the margin of the valve, two elevations with a limited perforated plate (ocellus) on each valve, and radial rows of poroid areolae with domed cribum-like veta, with a central granule, ornamenting the valve surface. It also shows many to just a few siliceous radial ribs located between the border of the elevated central area and the mantle, twinned pores and spines relatively well developed and scattered over the valve. There are one or two labiate processes located next to the border of the central area and opposite to the elevations. These are sessile internally but have stout external tubes of varying length.

AMPHITETRAS C.G. Ehrenberg 1840

Amphitetras antediluviana C.G. Ehrenberg 1840

Ref. illus.: Jahn, R.; Kusber, W.H. 2006, p. 528, Figures 1-3.

Size: $35-45 \mu \mathrm{m}$ long, $38 \mu \mathrm{m}$ broad, areolae $4-6$ in $10 \mu \mathrm{m}(n=2)$ (Figure 15a,b).

PSEUDICTYOTA P.A. Sims and D.M. Williams 2018

Pseudictyota dubia (T. Brightwell) P.A. Sims and D.M. Williams 2018

Ref. illus.: Hustedt, F. 1930, p. 806, Figure 46; Witkowski, A.; Lange-Bertalot, H.; Metzeltin, D. 2000, p. 42, pl. 8, Figures 4 and 5 (both as Triceratium dubium)

Size: $13-31 \mu \mathrm{m}$ long, areolae $4-5$ in $10 \mu \mathrm{m}(n=4)$ (Figure 13e-k).

Order: Coscinodiscales F.E. Round and R.M. Crawford 1990

Family Aulacodiscaceae (F. Schütt) E. Lemmermann

AULACODISCUS C.G. Ehrenberg, 1844, nom. cons.

Aulacodiscus sp.

Size: $65 \mu \mathrm{m}$ long, pervalvar axis $31 \mu \mathrm{m}(n=1)$ (Figure 16i,j).

Remark: Because no valve view was found for this taxon, the characteristics for its specific identification could not be used. However, elements in girdle view are indicative of the genus Aulacodiscus.

Family: Coscinodiscaceae F.T. Kützing 1844

COSCINODISCUS C.G. Ehrenberg 1839

Coscinodiscus gigas C.G. Ehrenberg 1841

Ref. illus.: Peragallo, H.; Peragallo, M. 1897-1908, p. 433, pl. 118, Figure 3.

Diameter $246 \mu \mathrm{m}$, areolae 3 in $10 \mu \mathrm{m}(n=2)$ (Figure 5g,h).

Coscinodiscus mesoleius P.T. Cleve $1883^{*,+}$

Ref. illus.: Cleve, P.T. 1883, p. 503, pl. 38, Figure 8; Stidolph, S.R.; Sterrenburg, F.A.S.; Smith, K.E.L.;

Kraberg, A. 2012, pl. 15, Figure 32, pl. 17, Figure 98.

Diameter 63-77 $\mu \mathrm{m}$, areolae 12 to 13 in $10 \mu \mathrm{m}(n=2)$ (Figure 6j,k). 


\section{Coscinodiscus radiatus C.G. Ehrenberg 1840}

Ref. illus.: Ehrenberg, C.G. 1840, p. 68, pl. 3, Figure 1a-c; Stidolph, S.R.; Sterrenburg, F.A.S.; Smith, K.E.L.; Kraberg, A. 2012, pl. 20, Figure 22; pl. 34, Figure 2, pl. 36, Figure 36, pl. 43, Figures 110 and 111; pl. 44, Figure 2; pl. 46, Figures 1 and 4.

Diameter 54-137 $\mu \mathrm{m}$, areolae 3-6 in $10 \mu \mathrm{m}(n=3)$ (Figures 5a-f and $6 \mathrm{k}, \mathrm{l})$.

Family: Heliopeltaceae H.L. Smith 1872

ACTINOPTYCHUS C.G. Ehrenberg 1843

Actinoptychus minutus R.K. Greville 1866

Ref. illus.: Greville, R.K. 1866, p. 5, pl. 1, Figure 12; Siqueiros Beltrones, D.A.; Argumedo-Hernández, U. 2015, p. 114, Figure 28.

Diameter $18-25 \mu \mathrm{m}(n=2)$ (Figure $3 \mathrm{e}-\mathrm{j})$.

\section{Actinoptychus senarius (C.G. Ehrenberg) C.G. Ehrenberg 1843}

Ref. illus.: Ehrenberg, C.G. 1843, p. 400, pl. 1.1, Figure 27; pl. 1.3, Figure 21; Siqueiros Beltrones, D.A.; Argumedo-Hernández, U. 2015, p. 114, Figure 8.

Diameter 28-46 $\mu \mathrm{m}(n=4)$ (Figure $4 \mathrm{a}-\mathrm{j})$.

\section{Actinoptychus vulgaris J. Schumann 1867}

Ref. illus.: Desikachary, T.V. 1988, p. 2, pl. 420, Figures 4 and 6; Moreno, J.L.; Licea, S.; Santoyo, H. 1996, p. 19, pl. 8, Figure 1; López-Fuerte, F.O.; Siqueiros Beltrones, D.A.; Navarro, J.N. 2010, p. 17, pl. 10, Figures 1 and 2.

Diameter 35-54 $\mu \mathrm{m}(n=4)$ (Figure 3a-d).

ACTINOCYCLUS C.G. Ehrenberg 1837

Actinocyclus subtilis (W.W. Gregory) J. Ralfs 1861

Ref. illus.: Witkowski, A.; Lange-Bertalot, H.; Metzeltin, D. 2000, p. 22, pl. 4, Figure 1; López-Fuerte, F.O.; Siqueiros Beltrones, D.A.; Navarro, J.N. 2010, p. 14, pl. 6, Figures 1 and 2.

Diameter 31-50 $\mu \mathrm{m}(n=5)$ (Figure 9a-e).

Actinocyclus tenuissimus P.T. Cleve 1878

Ref. illus.: Navarro, J.N. 1981, p. 429, Figures 28 and 29; Lobban, C.S.; Schefter, M.; Jordan, R.W.; Arai, Y.; Sasaki, A.; Theriot, E.C.; Ashworth, M.; Ruck, E.C.; Pennesi C. 2012, p. 249, pl. 5, Figures 1 and 2.

Diameter 38-58 $\mu \mathrm{m}$, areolae 18 in $10 \mu \mathrm{m}(n=2)$ (Figure 9f-h).

Actinocyclus ochotensis A.P. Jousé 1969 *

Ref. illus.: Jousé, A.P. 1969, p. 17, pl. 2, Figures 2-5.

Diameter $34 \mu \mathrm{m}$, areolae 8 in $10 \mu \mathrm{m}(n=1)$ (Figure $9 \mathrm{i}-\mathrm{k})$.

AZPEITIA M. Peragallo 1912

Azpeitia nodulifera (A.W.F. Schmidt) G.A. Fryxell and P.A. Sims 1986

Ref. illus.: Fryxell, G.A.; Sims, P.A.; Watkins, T.P. 1986, p. 19, Figures 17, 18 and 30; Hasle, G.R.; Syvertsen, E.E. 1996, p. 126, pl. 21.

Diameter 20-58 $\mu \mathrm{m}$, areolae 4-6 in $10 \mu \mathrm{m}(n=4)$ (Figure 7a-g).

Family: Hemidiscaceae N.I. Hendey, 1937 emend R. Simonsen, 1975

ROPERIA A. Grunow 1889

Roperia tesselata (F.C.S. Roper) A. Grunow 1889

Ref. illus.: Roper, F.C.S. 1858, p. 19, pl. 3, Figure 1; Hasle, G.R.; Syvertsen, E.E. 1996, p. 130, pl. 22.

Diameter $31-35 \mu \mathrm{m}$, areolae 7 to 8 in $10 \mu \mathrm{m}(n=2)$ (Figure $7 \mathrm{~m}$ ). 
Order: Melosirales R.M. Crawford 1990

Family: Hyalodiscaceae R.M. Crawford 1990

MARGARITUM H. Moreira Filho 1968

Margaritum terebro (G. Leuduger-Fortmorel) H. Moreira Filho 1968 *

Ref. illus.: Hendey, N.I. 1957, p. 36, pl. 1, Figures 12 and 13 (as Podosira tenebro); Souza-Mosimann, R.M. de; Fernandes, L.F.; Ludwig, T.V. 1997, p. 46, Figures 1-14.

Diameter 30-32 $\mu \mathrm{m}(n=2)$ (Figure 61-o).

PODOSIRA C.G. Ehrenberg 1840

Podosira montagnei F.T. Kützing 1844

Ref. illus.: Foged, N. 1984, p. 89, pl. 18, Figure 1.

Diameter $23 \mu \mathrm{m}(n=1)$ (Figure 8o).

\section{Podosira stelligera (J.W. Bailey) A.Mann 1907}

Ref. illus.: Hendey, N.I. 1964, p. 90, pl. 22, Figure 6; Navarro, J.N. 1982, p. 11, pl. 2, Figures 4 and 5. Diameter $17-42 \mu \mathrm{m}(n=6)$ (Figure $8 \mathrm{a}-\mathrm{k})$.

\section{Podosira variegata A.W.F. Schmidt 1889 *}

Ref. illus.: Schmidt, A.W.F. 1889, pl. 140, Figure 4.

Diameter $34 \mu \mathrm{m}(n=1)$ (Figure 81-n).

Family: Melosiraceae F.T. Kützing 1844

MELOSIRA C.A. Agardh 1824

Melosira moniliformis var. octogona (A. Grunow) F. Hustedt 1927

Ref. illus.: Witkowski, A.; Lange-Bertalot, H.; Metzeltin, D. 2000, p. 35, pl. 3, Figures 1 and 2.

Diameter $22 \mu \mathrm{m}(n=1)$ (Figure 6h,i).

Order: Paraliales R.M. Crawford 1990

Family: Paraliaceae R.M. Crawford 1988

PARALIA P.A.C. Heiberg 1863

Paralia sulcata (C.G. Ehrenberg) P.T. Cleve 1873

Ref. illus.: Hendey, N.I. 1964, p. 73, pl. 23, Figure 5; Hasle, G.R.; Syvertsen, E.E. 1996, p. 91, pl. 14.

Diameter 11-37 $\mu \mathrm{m}(n=3)$ (Figure 10g-1).

Family: Stephanodiscaceae I.V.Makarova 1986

CYCLOTELLA (F.T. Kützing) A. de Brébison 1838

Cyclotella striata (F.T. Kützing) A. Grunow 1880

Ref. illus.: Foged, N. 1984, p. 31, pl. 17 Figure 4; Hasle G.R., Syvertsen E.E. 1996, p. 34, pl. 1.

Diameter $15 \mu \mathrm{m}(n=1)$ (Figure 10f).

CYCLOSTEPHANOS F.E.Round 1987

Cyclostephanos sp.

Diameter $23 \mu \mathrm{m}(n=1)$ (Figure 10d).

Remark: According to [27], the distinctive characteristics of this genus are-circular valves with concentric undulations and spines around the margin; valves with radiating lines of areolae, grouped into fascicles around the margin and separated by raised, rounded ridges. The central region of the valve face variously has nodules or is plain. Cyclostephanos differs from Cyclotella in the extension of the rows of fascicles to the center of the valve and the presence of a single ring of spines around the valve face. 
Order: Thalassiosirales Z.I. Glezer and I.V. Makarova 1986

Family: Thalassiosiraceae M.V. Lebour 1930

THALASSIOSIRA P.T. Cleve 1873

Thalassiosira decipiens (A. Grunow ex H. Van Heurck) E.G. Jørgensen 1905

Ref. illus.: Navarro, J.N. 1982, p. 10, pl. 1, Figures 1 and 2; Moreno, J.L.; Licea, S.; Santoyo, H. 1996, p. 133, pl. 33, Figure 7.

Diameter 8-13 $\mu \mathrm{m}$, areolae 9-14 in $10 \mu \mathrm{m}(n=2)$ (Figure 6f,g).

\section{Thalassiosira eccentrica (C.G. Ehrenberg) P.T. Cleve 1904}

Ref. illus.: Hendey, N.I. 1964, p. 80, pl. 24, Figure 7; Navarro, J.N. 1982, p. 10, pl. 1, Figures 3 and 4; Moreno, J.L.; Licea, S.; Santoyo, H. 1996, p. 133, pl. 33, Figures 8 and 9.

Diameter $23 \mu \mathrm{m}$, areolae 8 in $10 \mu \mathrm{m}(n=1)$ (Figure 6e).

\section{Thalassiosira lineata A.P. Jousé 1968}

Ref. illus.: Hasle, G.R.; Syvertsen, E.E. 1996, p. 80, pl. 10; Park, J.S.; Jung, S.W.; Lee, S.D.; Yun, S.M.; Lee, J.H. 2016, p. 410, Figure 19.

Diameter 21-22 $\mu \mathrm{m}$, areolae 11-12 in $10 \mu \mathrm{m}(n=2)$ (Figure 6c,d).

\section{Thalassiosira leptopus (A. Grunow) G.R. Hasle and G.A. Fryxell 1977}

Ref. illus.: Hasle, G.R.; Fryxell, G.A. 1977, Figures 1-14; Hasle, G.R.; Syvertsen, E.E. 1996, pl. 10; Moreno, J.L.; Licea, S.; Santoyo, H. 1996, p. 134, pl. 33, Figure 11.

Diameter $108 \mu \mathrm{m}$, areolae 4 in $10 \mu \mathrm{m}(n=1)$ (Figure $6 \mathrm{a}, \mathrm{b}$ ).

\section{Thalassiosira nanolineata (A. Mann) G.A. Fryxell and G.R. Hasle 1977}

Ref. illus.: Mann, A. 1925, p. 68, pl. 14, Figure 4 (as Coscinodiscus nano-lineatus); Javeed, A.; Salleh, S.; Darif, A.; Mohammad, M. 2018, p. 8, Figure 2.

Diameter $19 \mu \mathrm{m}$, areolae 11 in $10 \mu \mathrm{m}(n=1)$ (Figure $7 \mathrm{~h}-\mathrm{j}$ ).

Order: Plagiogrammales E.J. Cox 2015

Family: Plagiogrammaceae G. De Toni 1890

DIMEREGRAMMA J. Ralfs 1861

Dimeregramma fulvum (W. Gregory) J. Ralfs 1861 *,

Ref. illus.: Witkowski, A.; Lange-Bertalot, H.; Metzeltin, D. 2000, p. 28, pl. 11, Figures 1 and 2.

Size: $43 \mu \mathrm{m}$ long, $8 \mu \mathrm{m}$ broad, 9 to 10 striae in $10 \mu \mathrm{m}(n=1)$ (Figure $17 \mathrm{c}, \mathrm{d})$.

\section{Dimeregramma minor (W. Gregory) J. Ralfs 1861}

Ref. illus.: Hendey, N.I. 1964, p. 156, pl. 27, Figure 12; Navarro, J.N.1982, p. 34, pl. 11, Figures 1-3; Witkowski, A.; Lange-Bertalot, H.; Metzeltin, D. 2000, p. 29, pl. 11, Figures 3-9.

Size: $15-32 \mu \mathrm{m}$ long, 7 to $8 \mu \mathrm{m}$ broad, 9 to 10 striae in $10 \mu \mathrm{m}(n=3)$ (Figure 17e-j).

TALARONEIS W.H.C.F. Kooistra and M. De Stefano 2004

PLAGIOGRAMMA R.K. Greville 1859

\section{Talaroneis sp.}

Ref. illus.: Navarro, J.N. 1982, p. 23, pl. 13, Figures 1 and 2; López-Fuerte, F.O.; Siqueiros Beltrones, D.A.; Navarro, J.N. 2010, p. 19, pl. 13, Figures 4-7 (both as Plagiogramma interruptum).

Size: $25-38 \mu \mathrm{m}$ long, $5-6 \mu \mathrm{m}$ broad, 20 striae in $10 \mu \mathrm{m}(n=2)$ (Figure 16a-c).

Remark: This taxon shows the combined characteristics of Plagiogramma and Talaroneis, but it is quite different from both of the three described species of the latter [32,33]. Plagiogramma interruptum in [34] on the other hand, is very similar to our specimen, structurally and morphometrically. In [33], the diagnostic characters of Talaroneis are: pores in an apical pore field, round two silica flaps parallel to the valve margin proximal to the apical pore fields. While [9] exhibits Talaroneis sp. (Figures 9 and 
10) that appears identical to our specimens, albeit thinner, but with clear subapical furrows as in ours that, in contrast with other described species of Talaroneis, show a stauros and apparent septa in the central area. The authors of [9] suggest that Talaroneis sp., may be a new species; in our case given the similarity with P. interruptum as in [34], p. 23, pl. 13, Figures 1, 2 and [35], p. 19, pl. 13, Figures 4-7, a review at the species and genus level may be required.

GLYPHODESMIS R.K. Greville 1862

Glyphodesmis rhombica (P.T. Cleve) R. Simonsen 1974 *

Ref. illus.: Mann, A. 1925, p. 78, pl. 16, Figures 2 and 3 (as G. acus); Simonsen, R. 1974, p. 35, pl. 23, Figure 1; Foged, N. 1978, p. 67, pl. 7, Figure 20.

Size: $26-48 \mu \mathrm{m}$ long, 9 to $10 \mu \mathrm{m}$ broad, 14 to 15 striae in $10 \mu \mathrm{m}(n=3)$ (Figure 25a-j).

Order: Triceratiales F.E. Round and R.M. Crawford 1990

Family: Triceratiaceae (F. Schütt) E. Lemmermann 1899

RALFSIELLA P.A. Sims, D.M. Williams and M. Ashworth 2018

Ralfsiella smithii (J. Ralfs) P.A. Sims, D.M. Williams and M. Ashworth 2018

Ref. illus.: Peragallo, H.; Peragallo, M. 1897-1908, p. 398, pl. 112, Figures 4 and 5; Hustedt, F. 1930, p. 861, Figure 513 (all as Cerataulus smithii); Sims, P.A., Williams, D.M.; Ashworth, M. 2018, p. 42, Figures 148-151.

Diameter $44 \mu \mathrm{m}, 9$ to 10 areolae in $10 \mu \mathrm{m}(n=1)$ (Figure $11 \mathrm{~g}-\mathrm{j})$.

TRICERATIUM C.G. Ehrenberg 1839

Triceratium balearicum f. biquadrata (C. Janisch) F. Hustedt 1930

Ref. illus.: Schmidt, A.W.F. 1886, pl. 98, Figures 4-6; Hustedt, F. 1930, p. 815, Figure 477.

Size: $33-86 \mu \mathrm{m}$ long $(n=6)$ (Figure $15 \mathrm{c}-\mathrm{g})$.

CLASS: FRAGILARIOPHYCEAE F.E. Round 1990

Order: Ardissoneales F.E. Round 1990

Family: Ardissoneaceae F.E. Round 1990

ARDISSONEA G. De Notaris 1870

Ardissonea formosa (C.A. Hantzsch) A. Grunow 1880

Ref. illus.: Witkowski, A.; Lange-Bertalot, H.; Metzeltin, D. 2000, p. 43, pl. 30, Figure 12.

Size: $331 \mu \mathrm{m}$ long, $18-20 \mu \mathrm{m}$ broad, 9 striae in $10 \mu \mathrm{m}(n=2)$ (Figure 18a).

Order: Climacospheniales F.E. Round 1990

Family: Climacospheniaceae F.E. Round 1990

CLIMACOSPHENIA C.G. Ehrenberg 1841

Climacosphenia elongata J.W. Bailey 1854

Ref. illus.: Round, F.E. 1982, Figures 4-6, 14; Stidolf, S.R.; Sterrengurg, F.A.S.; Smith K.E.L.; Kraberg, A. 2012, pl. 7, Figure 152, pl. 16, Figure 93; Al-Handal, A.Y.; Compère, P.; Riaux-Gobin, C. 2016, p. 12, pl. 6, Figures 1 and 2.

Size: $300 \mu \mathrm{m}$ long, $18 \mu \mathrm{m}$ broad, striae 36 in $10 \mu \mathrm{m}(n=1)$ (Figure 18b).

Order: Cyclophorales F.E. Round and R.M. Crawford 1990

Family Cyclophoraceae F.E. Round and R.M. Crawford 1990

CYCLOPHORA F. Castracane 1878

Cyclophora tenuis F. Castracane 1878

Ref. illus.: Peragallo, H.; Peragallo, M. 1897-1908, pl. 1, Figures 27-32; Navarro, J.N.1982, Figures 9-17; Navarro, J.N.; Lobban, C.S. 2009, Figures 59 and 60; Lobban, C.S.; Jordan, R.W. 2010, Figure 5b; Ashworth, M.P.; Ruck, E.C.; Lobban, C.S.; Romanovicz, D.K.; Theriot, E.C. 2012, p. 686, Figures 1, 5, 6 and $14-18$. 
Size: $52 \mu \mathrm{m}$ long, $12 \mu \mathrm{m}$ broad, striae not resolvable $(n=1)$ (Figure 37i).

Order: Fragilariales P.C. Silva 1962

Family: Fragilariaceae R.K. Greville 1833

OPEPHORA P. Pettit 1888

Opephora pacifica (A. Grunow) P. Pettit 1888

Ref. illus.: 1995, p. 244, Figures 45-53; Witkowski, A.; Lange-Bertalot, H.; Metzeltin, D. 2000 p. 72, pl. 25, Figures 18-26; Al-Handal, A.Y.; Compère, P.; Riaux-Gobin, C. 2016, p.8, pl. 4, Figures 12 and 13.

Size: $15-23 \mu \mathrm{m}$ long, 5 to $6 \mu \mathrm{m}$ broad, striae 9 in $10 \mu \mathrm{m}(n=2)$ (Figure 16a-c).

GEDANIELLA Chunlian Li, A.Witkowski and M.P.Ashworth 2018

Gedaniella mutabilis Chunlian Li and A.Witkowski, nom. illeg. 2018

Ref. illus.: Sabbe, K.; Vyverman, W. 1995, p. 241, Figures 13-28; Witkowski, A.; Lange-Bertalot, H.; Metzeltin, D. 2000, p. 72, pl. 25, Figures 10-17; Al-Handal, A.Y.; Compère, P.; Riaux-Gobin, C. 2016, p. 7, pl. 4, Figure 11.

Size: $15 \mu \mathrm{m}$ long, $4 \mu \mathrm{m}$ broad, striae 9 in $10 \mu \mathrm{m}(n=1)$ (Figure 16k).

STAUROSIRELLA D.M. Williams and F.E. Round 1987

Staurosirella guenter-grassii (A. Witkowski and H. Lange-Bertalot) E.A. Morales, C.E. Wetzel and L. Ector 2019 *

Ref. illus.: Sabbe, K.; Vyverman, W. 1995, p. 2, Figures 29-42, 66-71; Witkowski, A.; Lange-Bertalot, H.; Metzeltin, D. 2000, p. 70, pl. 24, Figures 40-44 (both as Opephora guenter-grassii).

Size: $8 \mu \mathrm{m}$ long, $3 \mu \mathrm{m}$ broad, striae 14 in $10 \mu \mathrm{m}(n=1)$ (Figure 161).

SYNEDRA C.G. Ehrenberg 1830

Synedra gaillonii var. macilenta cf. (A. Grunow) H. Peragallo 1881 *

Ref. illus.: Van Heurck, H. 1881, pl. 40, Figure 1.

Size: 139-148 $\mu \mathrm{m}$ long, $8 \mu \mathrm{m}$ broad $(n=2)$ (Figure 20a,b).

Synedra tabulata var. rostrata (H. Juhlin-Dannfelt) A. Cleve-Euler 1953 *

Ref. illus.: Juhlin-Dannfelt, H. 1882, p. 43, pl. 3, Figures 29 and 31 (as S. affinis var. rostrata); Cleve-Euler, A. 1953, p. 71, Figure 392g-h (as S. tabulata $\Lambda$ hybrida).

Size: $33-38 \mu \mathrm{m}$ long, 4 to $5 \mu \mathrm{m}$ broad, striae 15 to 16 in $10 \mu \mathrm{m}(n=3)$ (Figure 20m-o).

HYALOSYNEDRA D.M. Williams and F.E. Round 1986

Hyalosynedra laevigata (A. Grunow) D.M. Williams and F.E. Round 1986

Ref. illus.: Foged, N. 1984, p. 97, pl. 28, Figure 13; Witkowski, A.; Lange-Bertalot, H.; Metzeltin, D. 2000, p. 62, pl. 17, Figure 22; pl. 29, Figures 6-10; pl. 30, Figures 30-23.

Size: $204 \mu \mathrm{m}$ long, $6 \mu \mathrm{m}$ broad, striae not resolvable $(n=1)$ (Figure 20c).

FRAGILARIA H.C. Lyngbye 1819

Fragilaria barbatula (F.T. Kützing) H. Lange-Bertalot 1993

Ref. illus.: Witkowski, A.; Lange-Bertalot, H.; Metzeltin, D. 2000, p. 79, pl. 30, Figures 13 and 14 (as Synedra barbatula).

Size: 35-73 $\mu \mathrm{m}$ long, 4-7 $\mu \mathrm{m}$ broad, striae 18-20 in $10 \mu \mathrm{m}(n=7)$ (Figure 20j,p,q).

TABULARIA (F.T. Kützing) D.M. Williams and F.E. Round 1986

Tabularia fasciculata (C.A. Agardh) D.M. Williams and F.E. Round 1986

Ref. illus.: Williams, D.M.; Round, F.E. 1986, p. 326; Witkowski, A.; Lange-Bertalot, H.; Metzeltin, D. 2000, p. 80, pl. 30, Figures 4 and 5 (as Synedra fasciculata).

Size: $30 \mu \mathrm{m}$ long, $5 \mu \mathrm{m}$ broad, striae 17 to 18 in $10 \mu \mathrm{m}(n=1)$ (Figure 201). 
Tabularia investiens (W. Smith) D.M. Williams and F.E. Round 1986

Ref. illus.: Cleve-Euler, A. 1953, p. 44, Figure 354a-d (as Fragilaria investiens); Williams, D.M.; Round, F.E. 1986, p. 324, Figures 39-45.

Size: $45 \mu \mathrm{m}$ long, 3 to $4 \mu \mathrm{m}$ broad, striae 8 to 9 in $10 \mu \mathrm{m}(n=2)$ (Figure 20g,h,k).

\section{Tabularia parva (F.T. Kützing) D.M. Williams and F.E. Round 1986}

Ref. illus.: Williams, D.M.; Round, F.E. 1986, Figures 33-38; Lobban, C.S.; Schefter, M. 2012, p. 257, pl. 13, Figures 6 and 7.

Size: $35-55 \mu \mathrm{m}$ long, $4-7 \mu \mathrm{m}$ broad, striae $18-20$ in $10 \mu \mathrm{m}(n=4)$ (Figure 20i).

\section{Tabularia tabulata (C.A. Agardh) P.J.M. Snoeijs 1992}

Ref. illus.: Snoeijs, P.J.M. 1992, p. 343, Figures 38-48; Witkowski, A.; Lange-Bertalot, H.; Metzeltin, D. 2000, p. 81, pl. 30, Figures 1 and 2 (as Synedra tabulata var. tabulata).

Size: $113-134 \mu \mathrm{m}$ long, $5 \mu \mathrm{m}$ broad, striae 12 in $10 \mu \mathrm{m}(n=3)$ (Figure 20d-f).

Order: Licmophorales F.E. Round 1990

Family: Licmophoraceae F.T. Kützing 1844

LICMOPHORA C.A. Agardh 1827

\section{Licmophora abbreviata C.A. Agardh 1831}

Ref. illus.: Hustedt F. 1931-1959, p. 66, Figure 590; Witkowski, A.; Lange-Bertalot, H.; Metzeltin, D. 2000, p. 63, pl. 20, Figures 3-5.

Size: $87 \mu \mathrm{m}$ long, $21 \mu \mathrm{m}$ pervalvar axis, striae 16 in $10 \mu \mathrm{m}(n=1)$ (Figure 19h).

\section{Licmophora ehrenbergii (F.T. Kützing) A. Grunow 1867}

Ref. illus.: Hustedt, F. 1931-1959, p. 70, Figure 593; Witkowski, A.; Lange-Bertalot, H.; Metzeltin, D. 2000, p. 64, pls. 18, Figure 11; pl. 20, Figure 16.

Size: $72-145 \mu \mathrm{m}$ long, $25-31 \mu \mathrm{m}$ broad, striae 10-12 in $10 \mu \mathrm{m}(n=2)$ (Figure 19a,b).

\section{Licmophora gracilis var. anglica (F.T. Kützing) H. Peragallo and M. Peragallo 1901}

Ref. illus.: Peragallo, H.; Peragallo, M. 1901, p. 346, pl. 84. Figure 13; Witkowski, A.; Lange-Bertalot, H.; Metzeltin, D. 2000, p. 65, pl. 20, Figures 11-13.

Size: $23-28 \mu \mathrm{m}$ long, $5 \mu \mathrm{m}$ broad, striae 24 in $10 \mu \mathrm{m}(n=2)$ (Figure 19f,g).

\section{Licmophora paradoxa (H.C. Lyngbye) C.A. Agardh 1828}

Ref. illus.: Hustedt F. 1931-1959, p. 76, Figure 605; Witkowski, A.; Lange-Bertalot, H.; Metzeltin, D. 2000, p. 67, pl. 18, Figures 4-10.

Size: $69-75 \mu \mathrm{m}$ long, $8 \mu \mathrm{m}$ broad, striae 16 in $10 \mu \mathrm{m}(n=2)$ (Figure 19c,d).

\section{Licmophora pfannkucheae M.H. Giffen 1970}

Ref. illus.: Giffen, M.H. 1970, p. 278, Figures 41 and 42; Witkowski, A.; Lange-Bertalot, H.; Metzeltin, D. 2000 , p. 67 , pls. 18 , Figure 6.

Size: $108-119 \mu \mathrm{m}$ long, $6-8 \mu \mathrm{m}$ broad, striae 23 in $10 \mu \mathrm{m}(n=2)$ (Figure 19e).

Order: Rhabdonematales F.E. Round and R.M. Crawford 1990

Family: Rhabdonemataceae F.E. Round and R.M. Crawford 1990

HYALOSIRA F.T. Kützing 1844

Hyalosira tropicalis J.N. Navarro 1991

Ref. illus.: Navarro, J.N.; Williams, D.M. 1991, p. 328, Figures 1-14; Witkowski, A.; Lange-Bertalot, H.; Metzeltin, D. 2000, p. 62, pl. 21, Figures 16 and 17.

Size: 11 to $12 \mu \mathrm{m}$ long, 3-5 $\mu \mathrm{m}$ broad, striae $22-25$ in $10 \mu \mathrm{m}(n=2)$ (Figure 19i-k). 
RHABDONEMA F.T. Kützing 1844

Rhabdonema adriaticum F.T. Kützing 1844

Ref. illus.: Hustedt, F. 1931-1959, p. 23, Figure 552; Witkowski, A.; Lange-Bertalot, H.; Metzeltin, D. 2000, p. 76, pl. 13, Figures 10-12.

Size: $135-169 \mu \mathrm{m}$ long, striae 10 in $10 \mu \mathrm{m}(n=2)$ (Figure 16m,n).

Order: Rhaphoneidales F.E. Round 1990

Family: Psammodiscaceae F.E. Round and D.G. Mann 1990

PSAMMODISCUS F.E. Round and D.G. Mann 1980

Psammodiscus nitidus (W. Gregory) F.E. Round and D.G. Mann 1980

Ref. illus.: Hustedt, F. 1930, p. 414, Figure 221 (as Coscinodiscus nitidus); Witkowski, A.; Lange-Bertalot, H.; Metzeltin, D. 2000, p. 75, pl. 23, Figures 12-14.

Diameter $31 \mu \mathrm{m}$, areolae 5 in $10 \mu \mathrm{m}(n=1)$ (Figure 10e).

Family: Rhaphoneidaceae A. Forti 1912

DELPHINEIS G.W. Andrews 1977

Delphineis minutissima (F. Hustedt) R.R. Simonsen 1987

Ref. illus.: Hustedt, F. 1939, p. 599, Figures 14 and 15; Witkowski, A.; Lange-Bertalot, H.; Metzeltin, D. 2000, p. 45, pl. 22, Figures 11-14.

Size: $17 \mu \mathrm{m}$ long, $8 \mu \mathrm{m}$ broad, striae 13 in $10 \mu \mathrm{m}(n=1)$ (Figure 17k).

Family: Ulnariaceae E.J. Cox 2015

FALCULA M. Voigt 1960

Falcula media M. Voigt 1960

Ref. illus.: Voigt, M. 1960, p. 87; pl. 1, Figures 6-8; pl. 2, Figures 6-10.

Size: 63-69 $\mu \mathrm{m}$ long, 7 to $8 \mu \mathrm{m}$ broad $(n=3)$ (Figure 17a,b).

Order: Striatellales F.E. Round 1990

Family: Striatellaceae F.T. Kützing 1844

GRAMMATOPHORA C.G. Ehrenberg 1840

Grammatophora hamulifera F.T. Kützing 1844

Ref. illus.: Hustedt, F. 1931-1959, p. 40, Figure 566.

Size: $25 \mu \mathrm{m}$ long, $6 \mu \mathrm{m}$ broad, striae 16 in $10 \mu \mathrm{m}(n=4)$ (Figure 211,n-r).

\section{Grammatophora macilenta W. Smith 1856}

Ref. illus.: Hustedt, F. 1931-1959, p. 47, Figure 574; Witkowski, A.; Lange-Bertalot, H.; Metzeltin, D. 2000, p. 58, pl. 15, Figures 16-18.

Size: 98-108 $\mu \mathrm{m}$ long, $8 \mu \mathrm{m}$ broad, striae 22 in $10 \mu \mathrm{m}(n=7)$ (Figure 20r and Figure 21a,b).

\section{Grammatophora marina (H.C. Lyngbye) F.T. Kützing 1844}

Ref. illus.: Hustedt, F. 1931-1959, p. 43, Figure 569; Witkowski, A.; Lange-Bertalot, H.; Metzeltin, D. 2000, p. 58, pl. 15, Figures 9-12.

Size: $17-77 \mu \mathrm{m}$ long, 5 to $6 \mu \mathrm{m}$ broad, pervalvar axis $16-42 \mu \mathrm{m}$, striae $15-20$ in $10 \mu \mathrm{m}(n=14)$ (Figure 21k).

\section{Grammatophora oceanica C.G. Ehrenberg 1840}

Ref. illus.: Hustedt, F. 1931-1959, p. 45, Figure 573; Witkowski, A.; Lange-Bertalot, H.; Metzeltin, D. 2000, p. 59, pl. 15, Figures 13-14; pl. 16, Figure 12; pl. 17, Figures 3 and 4.

Size: 57-62 $\mu \mathrm{m}$ long, pervalvar axis $6 \mu \mathrm{m}$, striae 3 in $10 \mu \mathrm{m}(n=3)$ (Figure 21c-f,j). 
Grammatophora undulata var. gallopagensis A. Grunow

Ref. illus.: Van Heurck, H. 1880-1881, pl. 53, Figure 20; López-Fuerte, F.O.; Siqueiros Beltrones, D.A.; Jakes-Cota U.; Tripp-Valdéz, A. 2019, p.103, Figure 2h,i.

Size: $49-116 \mu \mathrm{m}$ long, $8-10 \mu \mathrm{m}$ broad, striae 27 to 28 in $10 \mu \mathrm{m}(n=5)$ (Figure $21 \mathrm{~g}-\mathrm{i}, \mathrm{m})$.

Order: Toxariales F.E. Round 1990

Family: Toxariaceae F.E. Round 1990

TOXARIUM J.W. Bailey 1854

Toxarium undulatum J.W. Bailey 1854

Ref. illus.: Hustedt, F. 1931-1959, p. 224, Figure 714; Witkowski, A.; Lange-Bertalot, H.; Metzeltin, D. 2000, p. 83, pl. 31, Figures 5 and 6.

Size: $400 \mu \mathrm{m}$ long, $3-8 \mu \mathrm{m}$ broad, striae $10-12$ in $10 \mu \mathrm{m}(n=3)$ (Figure $18 \mathrm{c}, \mathrm{d})$.

\section{CLASS: BACILLARIOPHYCEAE E. Haeckel 1878}

Order: Achnanthales P.C. Silva 1962

Family: Achnanthaceae F.T. Kützing 1844

ACHNANTHES J.B.G.M. Bory de Saint-Vincent 1822

Achnanthes cf. fimbriata (A. Grunow) R. Ross 1963

Ref. illus.: Witkowski, A.; Lange-Bertalot, H.; Metzeltin, D. 2000, p. 88, pls. 51, Figures 9 and 10; Siqueiros Beltrones, D.A.; Argumedo-Hernández, U.; López-Fuerte, F.O. 2017, p. 32, Figure 5b.

Size: $26-50 \mu \mathrm{m}$ long, $14-16 \mu \mathrm{m}$ broad, striae $12-16$ in $10 \mu \mathrm{m}(n=2)$ (Figure 22j, $, 1, \mathrm{~m})$.

\section{Achnanthes citronella (A. Mann) F. Hustedt 1937}

Ref. illus.: Riaux-Gobin, C. 2015, p. 104, Figures 15-17, 25, 26, 33, 35-38.

Size: Long, sternum valve $35-40 \mu \mathrm{m}$, raphe valve $41 \mu \mathrm{m}$. Broad; sternum valve $18-19 \mu \mathrm{m}$, raphe valve 20-22 $\mu \mathrm{m}$. Striae, sternum valve 11 in $10 \mu \mathrm{m}$, raphe valve 17-20 in $10 \mu \mathrm{m}(n=7)$ (Figure 22a-i).

Achnanthes groenlandica var. phinneyi C.D. McIntire and C.W. Reimer 1974

Ref. illus.: Mclntire, C.D.; Reimer, C.W. 1974, p. 172, pl. 2, Figure 3a-c; pl. 3, Figure 3a,b; Majewska, R.; De Stefano, M.; Ector, L.; Bolaños, F.; Frankovich, T.A.; Sullivan, M.J.; Ashworth, M.P.; Van de Vijver, B. 2017, p. 314, Figures 100-109.

Size: $43-54 \mu \mathrm{m}$ long, $7 \mu \mathrm{m}$ broad, striae 10 to 11 in $10 \mu \mathrm{m}(n=2)$ (Figure 22p,q).

\section{Achnanthes parvula F.T. Kützing 1844}

Ref. illus.: Hustedt, F. 1931-1959, p. 426, Figure 877f-i (as A. brevipes var. parvula); Witkowski, A.; Lange-Bertalot, H.; Metzeltin, D. 2000, p. 93, pl. 43, Figures 6 and 7; pl. 45, Figures 6-8, pl. 47, Figure 9. Size: $16-21 \mu \mathrm{m}$ long, $4-6 \mu \mathrm{m}$ broad, striae 10 to 11 in $10 \mu \mathrm{m}(n=2)$ (Figure 22n,o,u).

\section{Achnanthes pseudogroenlandica N.I. Hendey 1964}

Ref. illus.: Witkowski, A.; Lange-Bertalot, H.; Metzeltin, D. 2000, p. 94, pl. 44, Figures 16-23; Majewska, R.; De Stefano, M.; Ector, L.; Bolaños, F.; Frankovich, T.A.; Sullivan, M.J.; Ashworth, M.P.; Van de Vijver, B. 2017, p. 314, Figures 110-136.

Size: $25 \mu \mathrm{m}$ long, pervalvar axis $7 \mu \mathrm{m}$, striae 8 to 9 in $10 \mu \mathrm{m}(n=1)$ (Figure 22r,t).

\section{Achnanthes subconstricta (M. Meister) K. Toyoda 2003 *}

Ref. illus.: Toyoda, K.; Nagumo, T.; Osada, K.; Tanaka, J. 2003, p. 369; Lee, S.D.; Park, J.S.; Lee, J.H. 2011, p. 4, Figure 2G,N, Figure 5B-F.

Size: $38-75 \mu \mathrm{m}$ long, $14 \mu \mathrm{m}$ broad, striae $5-7$ in $10 \mu \mathrm{m}(n=2)$ (Figure 22k-v).

\section{Achnanthes yaquinensis C.D. McIntire and R.W. Reimer 1974}

Ref. illus.: Mclntire, C.D.; Reimer, R.W. 1974, p. 174, pls. 2, Figure 1a,b, pl. 3, Figure 1a,b. 
Size: $44 \mu \mathrm{m}$ long, $10 \mu \mathrm{m}$ broad, striae 9 to 10 in $10 \mu \mathrm{m}(n=1)$ (Figure 22s).

AMPHICOCCONEIS M. De Stefano and D. Marino 2002

Amphicocconeis discrepans (A.W.F. Schmidt) C. Riaux-Gobin, A. Witkowski, L. Ector and A. Igersheim 2018

Ref. illus.: Witkowski, A.; Lange-Bertalot, H.; Metzeltin, D. 2000, p. 106, pl. 41, Figures 35-40; pl. 42, Figures 26 and 27 (as Cocconeis discrepans); Riaux-Gobin, C., Ector, L., Witkowski, A. and Igersheim, A. 2018, p. 576, Figures 9-22.

Size: $19 \mu \mathrm{m}$ long, $8 \mu \mathrm{m}$ broad, striae 14 in $10 \mu \mathrm{m}(n=1)$ (Figure 25v).

Amphicocconeis disculoides (F. Hustedt) M. De Stefano and D. Marino 2003

Ref. illus.: Witkowski, A.; Lange-Bertalot, H.; Metzeltin, D. 2000, p. 106, pl. 42, Figures 28-33 (as Cocconeis disculoides); De Stefano, M.; Marino, D. 2003, p. 362, Figures 1-32.

Size: $15-16 \mu \mathrm{m}$ long, $8 \mu \mathrm{m}$ broad, striae $9-11$ in $10 \mu \mathrm{m}(n=2)$ (Figure 23s,t).

ASTARTIELLA A. Witkowski, H. Lange-Bertalot and D. Metzeltin 1998

Astartiella bahusiensis (A. Grunow) A. Witkowski, H. Lange-Bertalot and D. Metzeltin 1998 *

Ref. illus.: Witkowski, A.; Lange-Bertalot, H.; Stachura, K. 1998, p. 359, Figure 80: 1-3; Witkowski, A.; Lange-Bertalot, H.; Metzeltin, D. 2000, p. 99, pl. 52, Figures 22-31.

Size: $18 \mu \mathrm{m}$ long, $8 \mu \mathrm{m}$ broad, striae 24 in $10 \mu \mathrm{m}(n=1)$ (Figure 17p).

KARAYEVIA F.E. Round and L. Bukhtiyarova 1998

Karayevia amoena (F. Hustedt) L. Bukhtiyarova 1999

Ref. illus.: Chang, T.P. 1992, p. 401, Figures 3a-d, 4a-h, 5a-i; Witkowski, A.; Lange-Bertalot, H.; Metzeltin, D. 2000, p. 85, pl. 51, Figures 34-36 (both as Achnanthes amoena).

Size: $12 \mu \mathrm{m}$ long, $5 \mu \mathrm{m}$ broad, sternum valve striae $18-19$ in $10 \mu \mathrm{m}$, raphe valve 26 in $10 \mu \mathrm{m}(n=5)$ (Figure 17m-o).

PLANOTHIDIUM F.E. Round and L. Bukhtiyarova 1996

Planothidium campechianum (F. Hustedt) A. Witkowski, H. Lange-Bertalot and D. Metzeltin 2000

Ref. illus.: Hustedt, F. 1952, p. 389, Figures 87-90 (as Achnanthes campechianum); Witkowski, A.; Lange-Bertalot, H.; Metzeltin, D. 2000, p. 118, pl. 48, Figures 3-9.

Size: $26 \mu \mathrm{m}$ long, $8 \mu \mathrm{m}$ broad, striae 16 in $10 \mu \mathrm{m}(n=1)$ (Figure 25s).

Planothidium delicatulum (F.T. Kützing) F.E. Round and L. Bukhtiyarova 1996 *

Ref. illus.: Hustedt, F. 1931-1959, p. 389, Figure 836 (as Achnanthes delicatula); Witkowski, A.; Lange-Bertalot, H.; Metzeltin, D. 2000, p. 118, pl. 46, Figures 28 and 29; pl. 48, Figures 1 and 2.

Size: $17-21 \mu \mathrm{m}$ long, $9-10 \mu \mathrm{m}$ broad, sternum valve striae 9 in $10 \mu \mathrm{m}$, raphe valve 11 in $10 \mu \mathrm{m}(n=2)$ (Figure 25q,t,u).

Planothidium hauckianum (A. Grunow) F.E. Round and L. Bukhtiyarova 2008

Ref. illus.: Hustedt, F. 1931-1959, p. 388, Figure 834 (as Achnanthes hauckiana); Witkowski, A.; Lange-Bertalot, H.; Metzeltin, D. 2000, p. 120, pl. 48, Figures 39-41.

Size: $14-21 \mu \mathrm{m}$ long, 6 to $7.5 \mu \mathrm{m}$ broad, striae 10 to 11 in $10 \mu \mathrm{m}(n=5)$ (Figure $25 \mathrm{w}$ ).

Planothidium lilljeborgei (A. Grunow) A. Witkowski, H. Lange-Bertalot and D. Metzeltin 2000

Ref. illus.: Hustedt, F. 1931-1959, p. 394, Figure 843 (as Achnanthes lilljeborgei); Witkowski, A.; Lange-Bertalot, H.; Metzeltin, D. 2000, p. 121, pl. 49, Figure 1; pl. 51, Figures 27-29.

Size: $20 \mu \mathrm{m}$ long, $7 \mu \mathrm{m}$ broad, striae 10 in $10 \mu \mathrm{m}(n=1)$ (Figure 25p). 
Planothidium polare (E. Østrup) A. Witkowski, H. Lange-Bertalot and D. Metzeltin 2000

Ref. illus.: Witkowski, A.; Lange-Bertalot, H.; Metzeltin, D. 2000, p. 123, pl. 47, Figures 1-4; pl. 49, Figures 37-39 (as P. polaris).

Size: $42 \mu \mathrm{m}$ long, $14 \mu \mathrm{m}$ broad, striae 14 in $10 \mu \mathrm{m}(n=1)$ (Figure 25r).

Family: Cocconeidaceae F.T. Kützing 1844

COCCONEIS C.G. Ehrenberg 1838

Cocconeis californica A. Grunow 1880

Ref. illus.: Hustedt, F. 1931-1959, p. 343, Figure 796; Poulin, M.; Berard-Therriault, L.; Cardinal, A. 1984, p. 49, Figures 5-11.

Size: $10-16 \mu \mathrm{m}$ long, 5-10 $\mu \mathrm{m}$ broad, striae 15-17 in $10 \mu \mathrm{m}(n=5)$ (Figure 23q,r).

\section{Cocconeis contermina A.W.F. Schmidt 1894}

Ref. illus.: Schmidt, A.W.F. 1894, pl. 196, Figure 21; Siqueiros Beltrones, D.A.; Argumedo Hernández, U.; Landa Cansigno, C. 2016, p. 70, Figure 59.

Size: $38 \mu \mathrm{m}$ long, $28-30 \mu \mathrm{m}$ broad, striae 15-17 in $10 \mu \mathrm{m}(n=2)$ (Figure $24 \mathrm{c}-\mathrm{f})$.

\section{Cocconeis convexa M.H. Giffen 1967}

Ref. illus.: Witkowski, A.; Lange-Bertalot, H.; Metzeltin, D. 2000, p. 104, pls. 37, Figures 5 and 6; pl. 41, Figures 1-4. Sar, E.A.; Romero, O.E.; Sunesen, I. 2003, p.81, Figures 2-6.

Size: $18-28 \mu \mathrm{m}$ long, $12-22 \mu \mathrm{m}$ broad, sternum valve striae 28-47 in $10 \mu \mathrm{m}$, raphe valve 22 in $10 \mu \mathrm{m}$ $(n=4)$ (Figure 24g-j).

\section{Cocconeis dirupta W. Gregory 1857}

Ref. illus.: Kobayasi H.; Nagumo, T. 1985, p. 99, Figure 2: 16-27; Witkowski, A.; Lange-Bertalot, H.; Metzeltin, D. 2000, p. 105, pls. 39, Figures 1-5; pl. 51, Figures 5 and 8.

Size: $15-62 \mu \mathrm{m}$ long, 8-47 $\mu \mathrm{m}$ broad, sternum valve striae 18-27 in $10 \mu \mathrm{m}$, raphe valve striae 18-24 in $10 \mu \mathrm{m}(n=18)$ (Figure 23a-g).

\section{Cocconeis guttata F. Hustedt and A.A. Aleem 1951}

Ref. illus.: Witkowski, A.; Lange-Bertalot, H.; Metzeltin, D. 2000, p. 108, pl. 40, Figures 13-18; Sar, E.A.; Romero, O.E.; Sunesen, I. 2003, p. 86, Figures 16-21.

Size: $23-30 \mu \mathrm{m}$ long, $15-18 \mu \mathrm{m}$ broad, sternum valve striae 6 to 7 in $10 \mu \mathrm{m}(n=2)$ (Figure 24o,p).

\section{Cocconeis heteroidea C.A. Hantzsch 1863}

Ref. illus.: Hustedt, F. 1931-1959, p. 356, Figure 811; Witkowski, A.; Lange-Bertalot, H.; Metzeltin, D. 2000, p. 108, pl. 35, Figures 4 and 5.

Size: $38-45 \mu \mathrm{m}$ long, 28-31 $\mathrm{m}$ broad, sternum valve striae 23-26 in $10 \mu \mathrm{m}$, raphe valve striae 20 in $10 \mu \mathrm{m}(n=2)$ (Figure $23 \mathrm{k}-\mathrm{m})$.

\section{Cocconeis krammeri H. Lange-Bertalot and D. Metzeltin 1996}

Ref. illus.: Witkowski, A.; Lange-Bertalot, H.; Metzeltin, D. 2000, p. 109, pl. 33, Figures 1-5; pl. 34, Figures 4 and 5; pl. 42, Figure 34.

Size: $22-27 \mu \mathrm{m}$ long, 18-14 $\mu \mathrm{m}$ broad, sternum valve striae $25-30$ in $10 \mu \mathrm{m}$, raphe valve striae 23-28 in $10 \mu \mathrm{m}(n=2)$ (Figure $24 \mathrm{k}-\mathrm{n})$.

\section{Cocconeis lineata C.G. Ehrenberg 1849}

Ref. illus.: Ehrenberg, C.G. 1849, p. 301, pl. 5, Figure 44; p. 4, Romero, O.; Jahn, R. 2013, Figures 1-8. Size: $55 \mu \mathrm{m}$ long, $44 \mu \mathrm{m}$ broad, striae 17 to 18 in $10 \mu \mathrm{m}(n=1)$ (Figure 24a,b). 


\section{Cocconeis peltoides F. Hustedt 1939}

Ref. illus.: Witkowski, A.; Lange-Bertalot, H.; Metzeltin, D. 2000, p. 112, pl. 38, Figures 1-9; Sar, E.A.; Romero, O.E.; Sunesen, I. 2003, p. 91, Figures 34-41.

Size: $15-21 \mu \mathrm{m}$ long, 9-12 $\mu \mathrm{m}$ broad, sternum valve striae 13 in $10 \mu \mathrm{m}(n=2)$ (Figure $25 \mathrm{~m}, \mathrm{n})$.

\section{Cocconeis pseudomarginata W. Gregory 1857}

Ref. illus.: Hustedt, F. 1931-1959, p. 359, Figure 813; Witkowski, A.; Lange-Bertalot, H.; Metzeltin, D. 2000, p. 113, pl. 34, Figures 8 and 9; pl. 35, Figures 1-4.

Size: $42-56 \mu \mathrm{m}$ long, 32-36 $\mu \mathrm{m}$ broad, sternum valve striae 19-22 in $10 \mu \mathrm{m}$, raphe valve striae 19 in $10 \mu \mathrm{m}(n=2)$ (Figure 24q-t).

\section{Cocconeis scutellum C.G. Ehrenberg 1838}

Ref. illus.: Witkowski, A.; Lange-Bertalot, H.; Metzeltin, D. 2000, p. 114, pl. 36, Figures 1-7; pl. 38, Figure 11; Sar, E.A.; Romero, O.E.; Sunesen, I. 2003, p. 95, Figures 44-50.

Size: 29 to $30 \mu \mathrm{m}$ long, $18 \mu \mathrm{m}$ broad, sternum valve striae 8 in $10 \mu \mathrm{m}$, raphe valve striae $10-12$ in $10 \mu \mathrm{m}$ $(n=5)$ (Figure 25k,1).

\section{Cocconeis scutellum var. parva (A. Grunow) P.T. Cleve 1895}

Ref. illus.: Peragallo, H.; Peragallo, M. 1897-1908, p. 20, pl. 4, Figure 3; Poulin, M.; Berard-Therriault, L.; Cardinal, A. 1984, p. 56, Figures 49-53.

Size: $12-16 \mu \mathrm{m}$ long, $6-9 \mu \mathrm{m}$ broad, sternum valve striae $13-16$ in $10 \mu \mathrm{m}$, raphe valve striae 12 to 13 in $10 \mu \mathrm{m}(n=30)$ (Figure 23n-p).

Order: Bacillariales N.I. Hendey 1937

Family: Bacillariaceae C.G. Ehrenberg 1831

BACILLARIA J.F. Gmelin 1791

\section{Bacillaria socialis (W. Gregory) J. Ralfs 1861}

Ref. illus.: Poulin, M.; Berard-Therriault, L.; Cardinal, A. 1984, p. 75, Figures 2-4, 8; Witkowski, A.; Lange-Bertalot, H.; Metzeltin, D. 2000, p. 357, pl. 196, Figures 5-7; pl. 207, Figure 9.

Size: $57-156 \mu \mathrm{m}$ long, 7 to $8 \mu \mathrm{m}$ broad, fibulae 6 to 7 in $10 \mu \mathrm{m}$, striae 14 to 15 in $10 \mu \mathrm{m}(n=9)$ (Figure 44a,y,z).

CYMBELLONITZSCHIA F. Hustedt 1924

Cymbellonitzschia banzuensis J.G. Stepanek, S.E. Hamsher, S. Mayama, D.H. Jewson and J.P. Kociolek 2016

Ref. illus.: Stepanek, J.G., Hamsher, S.E., Mayama, S., Jewson, D.H. and Kociolek, J.P. 2016, p. 28, Figures 1-22.

Size: $15-38 \mu \mathrm{m}$ long, 2 to $3 \mu \mathrm{m}$ broad, fibulae $7-9$ in $10 \mu \mathrm{m}$, striae $15-17$ in $10 \mu \mathrm{m}(n=5)$ (Figure $44 \mathrm{n}-\mathrm{r})$.

CYLINDROTHECA L. Rabenhorst 1859

Cylindrotheca closterium (C.G. Ehrenberg) B.E.F. Reimann and J.C. Lewin 1964

Ref. illus.: Witkowski, A.; Lange-Bertalot, H.; Metzeltin, D. 2000, p. 374, pl. 212, Figures 4-6.

Size: $112-223 \mu \mathrm{m}$ long, $7-12 \mu \mathrm{m}$ broad, fibulae $12-37$ in $10 \mu \mathrm{m}(n=2)$ (Figure $45 \mathrm{j}$ ).

FRAGILARIOPSIS F. Hustedt 1913

Fragilariopsis doliolus (G.C. Wallich) L.K. Medlin and P.A. Sims 1993

Ref. illus.: Hasle, G.R.; Syvertsen, E.E.1996, p. 303, Figure 69; Witkowski, A.; Lange-Bertalot, H.; Metzeltin, D. 2000, p. 360, pl. 213, Figures 38 and 39.

Size: $50-65 \mu \mathrm{m}$ long, $8 \mu \mathrm{m}$ broad, striae 11 in $10 \mu \mathrm{m}(n=5)$ (Figure 25o). 
HANTZSCHIA A. Grunow 1877

Hantzschia marina (A.S. Donkin) A. Grunow 1880

Ref. illus.: Krammer, K.; Lange-Bertalot, H. 1988, p. 132, pl. 93, Figures 1-3; Witkowski, A.; Lange-Bertalot, H.; Metzeltin, D. 2000, p. 363, pl. 178, Figures 9-11.

Size: $32-86 \mu \mathrm{m}$ long, $5-8 \mu \mathrm{m}$ broad, fibulae $4-6$ in $10 \mu \mathrm{m}$, striae 7 to 8 in $10 \mu \mathrm{m}(n=2)$ (Figure $45 \mathrm{~d}-\mathrm{g})$.

NAGUMOEA J.P. Kociolek and A. Witkowski 2011

Nagumoea vallus (V.A. Nikolaev) R. Majewska and B. Van de Vijver 2020 *

Ref. illus.: Nikolaev, V.A.1969, p. 30, pl. 1, Figures 3-8 (as Anaulus vallus); Sullivan, M.J. 2010, p. 175, Figures 1, 2, 4-6, 7 and 8 (as Denticula vallus).

Size: $16-20 \mu \mathrm{m}$ long, $2 \mu \mathrm{m}$ broad, fibulae 5 in $10 \mu \mathrm{m}(n=2)$ (Figure $17 \mathrm{r}$ ).

NITZSCHIA A.H. Hassall 1845

Nitzschia agnita F. Hustedt 1957

Ref. illus.: Krammer, K.; Lange-Bertalot, H. 1988, p. 117, Figure 82: 1-5; Witkowski, A.; Lange-Bertalot, H.; Metzeltin, D. 2000, p. 367, pl. 210, Figures 22 and 23.

Size: $23 \mu \mathrm{m}$ long, $5 \mu \mathrm{m}$ broad, fibulae 17 in $10 \mu \mathrm{m}(n=1)$ (Figure $44 \mathrm{~m}$ ).

\section{Nitzschia amabilis H. Suzuki 2010}

Ref. illus.: Witkowski, A.; Lange-Bertalot, H.; Metzeltin, D. 2000, p. 387, pl. 189, Figures 13-15, pl. 190, Figures 1-6 (as Nitzschia laevis); Suzuki, H.; Nagumo, T.; Tanaka, J. 2010, p. 223, Figure 1.

Size: 8-12 $\mu \mathrm{m}$ long, 4 to $5 \mu \mathrm{m}$ broad, fibulae $10-14$ in $10 \mu \mathrm{m}(n=2)$ (Figure $44 \mathrm{u}, \mathrm{v})$.

\section{Nitzschia angularis W. Smith 1853}

Ref. illus.: Hendey, N.I. 1964, p. 281, Figure 39: 6; Witkowski, A.; Lange-Bertalot, H.; Metzeltin, D. 2000, p. 368, pl. 199, Figures 5 and 6.

Size: 142 to $143 \mu \mathrm{m}$ long, 13-15 $\mu \mathrm{m}$ broad, fibulae 4 in $10 \mu \mathrm{m}(n=2)$ (Figure 44f,g).

\section{Nitzschia bicapitata P.T. Cleve 1901}

Ref. illus.: Fryxell, G. 2000, p. 46, Figures 1-11.

Size: $24 \mu \mathrm{m}$ long, $5 \mu \mathrm{m}$ broad, fibulae 12 in $10 \mu \mathrm{m}$, striae 25 in $10 \mu \mathrm{m}(n=2)$ (Figure 44k,l).

\section{Nitzschia carnicobarica T.V. Desikachary and P. Prema 1987}

Ref. illus.: Desikachary, T.V.; Prema, P. 1987, p. 8, Figure 304: 5; Witkowski, A.; Lange-Bertalot, H.; Metzeltin, D. 2000, p. 373, pl. 183, Figures 9 and 10.

Size: $28-38 \mu \mathrm{m}$ long, $8-11 \mu \mathrm{m}$ broad, fibulae $11-12$ in $10 \mu \mathrm{m}(n=3)$ (Figure $42 \mathrm{~m}, \mathrm{n}$ and Figure 431$)$.

\section{Nitzschia composita M.H. Giffen 1971 *}

Ref. illus.: Giffen, M.H. 1971, p. 8, Figures 42 and 43; Witkowski, A.; Lange-Bertalot, H.; Metzeltin, D. 2000, p. 376, pl. 211, Figure 12.

Size: $42-45 \mu \mathrm{m}$ long, $7 \mu \mathrm{m}$ broad, fibulae $9-11$ in $10 \mu \mathrm{m}(n=2)$ (Figure $45 \mathrm{o}, \mathrm{p})$.

\section{Nitzschia costata J. Pantocsek 1892 *}

Ref. illus.: Pantocsek, J. 1892, pl. 41, Figure 566.

Size: $128 \mu \mathrm{m}$ long, $7-9 \mu \mathrm{m}$ broad, costae 5 in $10 \mu \mathrm{m}(n=1)$ (Figure $45 \mathrm{~s})$.

\section{Nitzschia distans W. Gregory 1857}

Ref. illus.: Peragallo, H.; Peragallo, M. 1897-1908, p. 283, pl. 73, Figure 3; Witkowski, A.; Lange-Bertalot, H.; Metzeltin, D. 2000, p. 378, pl. 203, Figures 7-9.

Size: $69 \mu \mathrm{m}$ long, $7 \mu \mathrm{m}$ broad, fibulae 4 in $10 \mu \mathrm{m}(n=1)$ (Figure $44 \mathrm{x}$ ). 


\section{Nitzschia frustulum (F.T. Kützing) A. Grunow 1880}

Ref. illus.: Krammer, K.; Lange-Bertalot, H. 1988, p. 94, pl. 68, Figures 1-9; Witkowski, A.; Lange-Bertalot, H.; Metzeltin, D. 2000, p. 382, pl. 209, Figures 13-17.

Size: $7-15 \mu \mathrm{m}$ long, $2 \mu \mathrm{m}$ broad, fibulae $10-16$ in $10 \mu \mathrm{m}(n=2)$ (Figure $44 \mathrm{~s}, \mathrm{t})$.

\section{Nitzschia fusiformis A. Grunow 1880 *}

Ref. illus.: Lange-Bertalot, H.; Krammer, K. 1987, p. 20, pl. 28, Figures 4-10; Witkowski, A.; Lange-Bertalot, H.; Metzeltin, D. 2000, p. 382, pl. 197, Figures 17-20; pl. 198, Figures 1-3.

Size: $108 \mu \mathrm{m}$ long, $5 \mu \mathrm{m}$ broad, fibulae 10 to 11 in $10 \mu \mathrm{m}$, striae 24 in $10 \mu \mathrm{m}(n=1)$ (Figure $45 \mathrm{a}$ ).

\section{Nitzschia gracilis C.A. Hantzsch 1860}

Ref. illus.: Lange-Bertalot, H. 1988, p. 93. pl. 66, Figures 1-11.

Size: $46-62 \mu \mathrm{m}$ long, 3 to $4 \mu \mathrm{m}$ broad, fibulae 11 to 12 in $10 \mu \mathrm{m}$, striae 26 in $10 \mu \mathrm{m}(n=3)$ (Figure 43p,q).

\section{Nitzschia hybrida A. Grunow 1880}

Ref. illus.: Krammer, K.; Lange-Bertalot, H. 1988, p. 61, pl. 46, Figures 3-6; Witkowski, A.; Lange-Bertalot, H.; Metzeltin, D. 2000, p. 385, pl. 191, Figures 12-14.

Size: $65-77 \mu \mathrm{m}$ long, 5-8 $\mu \mathrm{m}$ broad, fibulae 5-8 in $10 \mu \mathrm{m}(n=2)$ (Figure $45 \mathrm{k}, \mathrm{m})$.

\section{Nitzschia incrustans A. Grunow 1862 *}

Ref. illus.: Krammer, K.; Lange-Bertalot, H. 1988, p. 26, pl. 7, Figures 9 and 10a; Witkowski, A.; Lange-Bertalot, H.; Metzeltin, D. 2000, p. 386, pls. 203, Figures 1-3.

Size: $11-15 \mu \mathrm{m}$ long, 4 to $5 \mu \mathrm{m}$ broad, fibulae 5 to 6 in $10 \mu \mathrm{m}(n=3)$ (Figure $43 \mathrm{t}, \mathrm{u})$.

\section{Nitzschia incurva var. lorenziana R. Ross 1986}

Ref. illus.: Krammer, K.; Lange-Bertalot, H. 1988, p. 125, pl. 86, Figures 6-10; Witkowski, A.; Lange-Bertalot, H.; Metzeltin, D. 2000, p. 392, pls. 210, Figures 24 and 25; pl. 211, Figure 3; pl. 212, Figures 1-3.

Size: $138 \mu \mathrm{m}$ long, $7 \mu \mathrm{m}$ broad, fibulae 7 in $10 \mu \mathrm{m}$, striae 18 in $10 \mu \mathrm{m}(n=1)$ (Figure $45 \mathrm{~h})$.

\section{Nitzschia insignis W. Gregory 1857}

Ref. illus.: Peragallo, H.; Peragallo, M. 1897-1908, p. 295, pl. 75, Figure 5; Witkowski, A.; Lange-Bertalot, H.; Metzeltin, D. 2000, p. 387, pl. 202, Figure 5, pl. 204, Figures 1-7.

Size: $119 \mu \mathrm{m}$ long, $8 \mu \mathrm{m}$ broad, fibulae $2-5$ in $10 \mu \mathrm{m}$, striae $10-14$ in $10 \mu \mathrm{m}(n=4)$ (Figure 42d,e and Figure 45r).

\section{Nitzschia lanceolata W. Smith 1853}

Ref. illus.: Krammer, K.; Lange-Bertalot, H. 1988, pl. 16, Figures 1-8; Witkowski, A.; Lange-Bertalot, H.; Metzeltin, D. 2000, p. 389, pl. 194, Figures 1-5.

Size: $69 \mu \mathrm{m}$ long, $8 \mu \mathrm{m}$ broad, fibulae 10 in $10 \mu \mathrm{m}(n=1)$ (Figure $45 \mathrm{n})$.

\section{Nitzschia linearis W. Smith 1853}

Ref. illus.: Hustedt, F. 1930, p. 409, Figure 784.

Size: $100 \mu \mathrm{m}$ long, $5 \mu \mathrm{m}$ broad, fibulae 8 in $10 \mu \mathrm{m}(n=1)$ (Figure 45l).

\section{Nitzschia longa A. Grunow 1880}

Ref. illus.: Peragallo, H.; Peragallo, M. 1897-1908, p. 279, pl. 72, Figure 5; Stidolph, S.R.; Sterrenburg, F.A.S.; Smith, K.E.L.; Kraberg, A. 2012, pl. 25, Figure 84.

Size: $162 \mu \mathrm{m}$ long, $8 \mu \mathrm{m}$ broad, fibulae 4 in $10 \mu \mathrm{m}$, striae 12 to 13 in $10 \mu \mathrm{m}(n=1)$ (Figure $44 \mathrm{~b}, \mathrm{c}$ ). 


\section{Nitzschia longissima (A. Brébisson) A. Pritchard 1861}

Ref. illus.: Pritchard, A. 1861, p. 783, pl. 4, Figure 23; Witkowski, A.; Lange-Bertalot, H.; Metzeltin, D. 2000, p. 391, pl. 207, Figures 6 and 7.

Size: $200-500 \mu \mathrm{m}$ long, 5-8 $\mu \mathrm{m}$ broad, fibulae $6-10$ in $10 \mu \mathrm{m}(n=5)$ (Figure $45 \mathrm{i})$.

\section{Nitzschia martiana (Agardh) H. Van Heurck 1896}

Ref. illus.: Peragallo, H.; Peragallo, M. 1897-1908, p. 282, pl. 72, Figure 20; Lobban, C.S.; Mann, D.G. 1987, p. 2397, Figures 1-13.

Size: $240-262 \mu \mathrm{m}$ long, 3 to $4 \mu \mathrm{m}$ broad, fibulae 5 to 6 in $10 \mu \mathrm{m}(n=2)$ (Figure $45 \mathrm{t}$ ).

\section{Nitzschia sicula (F. Castracane) F. Hustedt 1958}

Ref. illus.: Hasle, G.R.; Syvertsen, E.E.1996, p. 327, pl. 75, Figures a-d.

Size: $30 \mu \mathrm{m}$ long, $7 \mu \mathrm{m}$ broad, striae and fibulae 10 in $10 \mu \mathrm{m}(n=1)$ (Figure $43 \mathrm{r}, \mathrm{s}$ ).

\section{Nitzschia sigma (F.T. Kützing) W. Smith 1853}

Ref. illus.: Krammer, K.; Lange-Bertalot, H. 1988, pl. 23, Figures 1-9; Witkowski, A.; Lange-Bertalot, H.; Metzeltin, D. 2000, p. 404, pl. 206, Figures 1-10.

Size: $69-77 \mu \mathrm{m}$ long, 6 to $7 \mu \mathrm{m}$ broad, fibulae 9 in $10 \mu \mathrm{m}$, striae 30 in $10 \mu \mathrm{m}(n=2)$ (Figure $45 \mathrm{~b}, \mathrm{c}$ ).

\section{Nitzschia spathulata W. Smith 1853}

Ref. illus.: Peragallo, H.; Peragallo, M. 1897-1908, p. 284, pl. 53, Figure 4.

Size: 50-135 $\mu \mathrm{m}$ long, 5-8 $\mu \mathrm{m}$ broad, fibulae 6 to 7 in $10 \mu \mathrm{m}(n=2)$ (Figure $44 \mathrm{~h}, \mathrm{w})$.

Nitzschia cf. spectabilis var. americana A. Grunow 1880 *

Size: $121 \mu \mathrm{m}$ long, $5 \mu \mathrm{m}$ broad, fibulae 8 in $10 \mu \mathrm{m}$, striae 25 in $10 \mu \mathrm{m}(n=1)$ (Figure $45 \mathrm{q})$.

Nitzschia valdestriata A.A. Aleem and F. Hustedt 1951

Ref. illus.: Krammer, K.; Lange-Bertalot, H. 1997, p. 121, pl. 84, Figures 9-12; Witkowski, A.; Lange-Bertalot, H.; Metzeltin, D. 2000, p. 407, pls. 203, Figures 19-21; pl. 207, Figures 14-16.

Size: $17 \mu \mathrm{m}$ long, $3 \mu \mathrm{m}$ broad, fibulae 8 in $10 \mu \mathrm{m}$, striae 14 to 15 in $10 \mu \mathrm{m}(n=1)$ (Figure 44i,j).

TRYBLIONELLA W. Smith 1853

\section{Tryblionella bathurstensis (M.H. Giffen) D.G. Mann 1990 *}

Ref. illus.: Giffen, M.H. 1970, 287 (as Nitzschia bathurstensis); Mann, D.G. 1990, p. 678.

Size: $18 \mu \mathrm{m}$ long, 8 to $9 \mu \mathrm{m}$ broad, fibulae 12 to 13 in $10 \mu \mathrm{m}$, striae 22 in $10 \mu \mathrm{m}(n=2)$ (Figure $43 \mathrm{e}, \mathrm{f}, \mathrm{i}$ ).

\section{Tryblionella coarctata (A. Grunow) D.G. Mann 1990}

Ref. illus.: Peragallo, H.; Peragallo, M. 1897-1908, p. 268, pl. 69, Figures 26 and 27 (as Nitzschia puncta var. coarctata).

Size: $15-44 \mu \mathrm{m}$ long, $7-16 \mu \mathrm{m}$ broad, striae 12 in $10 \mu \mathrm{m}(n=5)$ (Figure 43a-d,g,h).

\section{Tryblionella hungarica (A. Grunow) J. Frenguelli 1942}

Ref. illus.: Krammer, K.; Lange-Bertalot, H. 1988, p. 42, pl. 34, Figures 1-3; Witkowski, A.; Lange-Bertalot, H.; Metzeltin, D. 2000, p. 385, pl. 188, Figures 10 and 11 (as Nitzschia hungarica).

Size: 31-58 $\mu \mathrm{m}$ long, 6 to $7 \mu \mathrm{m}$ broad, fibulae 10 to 11 in $10 \mu \mathrm{m}$, striae 22 in $10 \mu \mathrm{m}(n=3)$ (Figure 43j,k).

\section{Tryblionella lanceola A. Grunow 1878}

Ref. illus.: Krammer, K.; Lange-Bertalot, H. 1988, pl. 38, Figures 11 and 12; Witkowski, A.; Lange-Bertalot, H.; Metzeltin, D. 2000, p. 388, pl. 212, Figures 13-17 (both as Nitzschia lanceola).

Size: $28 \mu \mathrm{m}$ long, $8 \mu \mathrm{m}$ broad, striae 9 to 10 in $10 \mu \mathrm{m}(n=1)$ (Figure $43 \mathrm{n}-\mathrm{o}$ ). 
Order: Cymbellales D.G. Mann 1990

Family: Rhoicospheniaceae J. Chen and H. Zhu 1983

RHOICOSPHENIA A. Grunow 1860

Rhoicosphenia abbreviata (C.A. Agardh) H. Lange-Bertalot 1980

Ref. illus.: Krammer, K.; Lange-Bertalot, H. 1986, p. 381, pl. 91, Figures 20-28; Witkowski, A.; Lange-Bertalot, H.; Metzeltin, D. 2000, p. 345, pl. 212, pl. 58, Figures 4-7.

Size: $25 \mu \mathrm{m}$ long, $4 \mu \mathrm{m}$ broad, striae 16 to 17 in $10 \mu \mathrm{m}(n=1)$ (Figure 38p).

GOMPHOSEPTATUM L.K. Medlin 1986

Gomphoseptatum aestuarii (P.T. Cleve) L.K. Medlin 1986

Ref. illus.: Medlin, L.K.; Round, F.E. 1986, p. 212, Figures 16-18; Witkowski, A.; Lange-Bertalot, H.; Metzeltin, D. 2000, p. 222, pl. 61, Figures 17 and 18.

Size: $21 \mu \mathrm{m}$ long, $3 \mu \mathrm{m}$ broad, striae 14 to 15 in $10 \mu \mathrm{m}(n=1)$ (Figure 17l).

Order: Eunotiales P.C. Silva 1962

Family: Eunotiaceae F.T. Kützing 1844

COLLICULOAMPHORA D.M. Williams and G. Reid 2006

Colliculoamphora reichardtiana (A. Grunow) Williams and Reid 2006

Ref. illus.: Williams, D.M., Reid, G. 2006, p. 153, Figures 11-19; Williams, D.M. 2016, p. 81, Figures 1-14. Size: $15 \mu \mathrm{m}$ long, $5 \mu \mathrm{m}$ broad, striae 15 in $10 \mu \mathrm{m}(n=1)$ (Figure 17q).

Remark: Our specimen is slightly shorter and narrower than in the references; $17-52 \mu \mathrm{m}$ long, $7-13 \mu \mathrm{m}$ broad, and may be mistaken for C. minima, but the number of striae in $10 \mu \mathrm{m}$ is 18 for this taxon.

Family: Lyrellaceae D.G.Mann 1990

LYRELLA N.I. Karayeva 1978

Lyrella approximatoides (F. Hustedt) D.G. Mann 1990

Ref. illus.: Hustedt, F. 1930-1966, p. 426, Figure 1498; Foged, N. 1984, p. 60, pl. 49, Figure 1 (both as Navicula approximatoides).

Size: $58 \mu \mathrm{m}$ long, $27 \mu \mathrm{m}$ broad, striae $8-9$ in $10 \mu \mathrm{m}(n=1)$ (Figure 28i,j).

\section{Lyrella atlantica (A.W.F. Schmidt) D.G. Mann 1990}

Ref. illus.: Hustedt, F. 1961-1966, p. 509, Figure 1555 (as Navicula lyra var. atlantica); Witkowski, A.; Lange-Bertalot, H.; Metzeltin, D. 2000, p. 231, pls. 96, Figure 6; pl. 98, Figure 5.

Size: $50 \mu \mathrm{m}$ long, $25 \mu \mathrm{m}$ broad, striae 11 in $10 \mu \mathrm{m}(n=1)$ (Figure 28h).

Lyrella clavata var. caribaea (P.T. Cleve) D.A. Siqueiros Beltrones 2017

Ref. illus.: Peragallo, H.; Peragallo, M. 1897-1908, p. 138, pl. 24, Figures 3 and 4 (as Navicula clavata var. caribaea); Siqueiros Beltrones, D.A.; Argumedo-Hernández, U.; López-Fuerte, F.O. 2017, Figures 2-12, $14-19,21$ and 22.

Size: $50 \mu \mathrm{m}$ long, 24 to $25 \mu \mathrm{m}$ broad, striae $11-12$ in $10 \mu \mathrm{m}(n=2)$ (Figure $28 \mathrm{~d}-\mathrm{g}$ ).

Lyrella hennedyi (W. Smith) A.J. Stickle and D. G. Mann 1990

Ref. illus.: Hustedt, F. 1961-1966, p. 453, Figure 1516; Witkowski, A.; Lange-Bertalot, H.; Metzeltin, D. 2000, p. 233, pl. 95, Figure 3; pl. 98, Figure 4.

Size: $77 \mu \mathrm{m}$ long, $38 \mu \mathrm{m}$ broad, striae 10 in $10 \mu \mathrm{m}(n=1)$ (Figure 28a-c).

Order: Mastogloiales D.G.Mann 1990

Family: Mastogloiaceae C. Mereschkowsky 1903

MASTOGLOIA G.H.K. Thwaites 1856

Mastogloia binotata (A. Grunow) P.T. Cleve 1895 
Ref. illus.: Moreno, J.L.; Licea, S.; Santoyo, H. 1996, p. 89, pl. 24, Figure 3; Witkowski, A.; Lange-Bertalot, H.; Metzeltin, D. 2000, p. 240, pl. 75, Figures 15-17.

Size: $22 \mu \mathrm{m}$ long, 14 to $15 \mu \mathrm{m}$ broad, striae 13 in $10 \mu \mathrm{m}(n=3)$ (Figure 28k-1).

Mastogloia chersonensis A.W.F. Schmidt 1893

Ref. illus.: Schmidt, A.W.F. 1893, pl. 186, Figures 31 and 32; Hustedt, F. 1933, p. 565, Figure 999a.

Size: $28 \mu \mathrm{m}$ long, $9 \mu \mathrm{m}$ broad $(n=1)$ (Figure 28o).

Mastogloia ciskeiensis M.H. Giffen 1967

Ref. illus.: Giffen, M.H. 1967, p. 264, Figures 43-45; Foged, N. 1975, p. 29, pl. 16, Figures 16 and 23; Foged, N. 1978, p. 78, pl. 18, Figure 7.

Size: $32 \mu \mathrm{m}$ long, $7 \mu \mathrm{m}$ broad $(n=1)$ (Figure $28 \mathrm{~m}, \mathrm{n}$ ).

TETRAMPHORA C. Mereschkowsky 1903

Tetramphora decussata (A. Grunow) J.G. Stepanek and J.P. Kociolek 2016

Ref. illus.: Peragallo, H.; Peragallo, M. 1897-1908, pl. 49, Figure 24; Lobban, C.S.; Schefter, M. 2012, p. 298, pl. 1, Figures 7-9; pl. 54, Figure 5; pl. 55, Figures 1-3 (both as Amphora decussata).

Size: $46 \mu \mathrm{m}$ long, $8 \mu \mathrm{m}$ broad, dorsal striae $13-15$ in $10 \mu \mathrm{m}$, ventral striae $21-22$ in $10 \mu \mathrm{m}(n=1)$ (Figure 36f-h).

Remark: Our specimen is smaller than other records, $60 \mu \mathrm{m}$ long and $10 \mu \mathrm{m}$ broad; the morphometrics coincide with that of Amphora acuta var. parva, but the dorsal striae are distinctively oblique relative to both raphe and fascia which differentiate our specimens from other records.

\section{Tetramphora intermedia (P.T. Cleve) J.G. Stepanek and J.P. Kociolek 2016}

Ref. illus.: Peragallo, H.; Peragallo, M. 1897-1908, p. 224, pl. 50, Figure 3, Wachnicka, A.H.; Gaiser, E.E. 2007, p. 419, Figure 118 (both as Amphora rhombica var. intermedia).

Size: $77-100 \mu \mathrm{m}$ long, $19 \mu \mathrm{m}$ broad, dorsal striae 13-19 in $10 \mu \mathrm{m}$, ventral striae 16 in $10 \mu \mathrm{m}(n=5)$ (Figure 36a-c).

Tetramphora securicula (H. Peragallo and M. Peragallo) J.G. Stepanek and J.P. Kociolek 2016 * Ref. illus.: Peragallo, H. and Peragallo, M. 1897-1908. p. 224, pl. 50, Figure 2.

Size: $62 \mu \mathrm{m}$ long, $15 \mu \mathrm{m}$ broad, dorsal striae 11 to 12 in $10 \mu \mathrm{m}$, ventral striae 14 in $10 \mu \mathrm{m}(n=1)$ (Figure 35p).

Remark: Our specimen is broader than the one reported in [36], who report a valve breadth of 8-10 $\mu \mathrm{m}$. However, the authors of [37] record it as $15 \mu \mathrm{m}$ wide and $70 \mu \mathrm{m}$ long, with 12 striae in $10 \mu \mathrm{m}$.

Order: Naviculales C.E. Bessey 1907

Family: Amphipleuraceae A. Grunow 1862

AMPHIPRORA C.G. Ehrenberg 1843

Amphiprora pseudoduplex (K. Osada and H. Kobayasi) G. Hällfors 2004

Ref. illus.: Osada, K.; Kobayasi, H. 1990, p. 165, Figures 4, 5 and 32-42 (as Entomoneis pseudoduplex).

Size: $25 \mu \mathrm{m}$ long, $7 \mu \mathrm{m}$ broad $(n=1)$ (Figure 37h).

HALAMPHORA (P.T. Cleve) Z. Levkov 2009

Halamphora acutiuscula (F.T. Kützing) Z. Levkov 2009

Ref. illus.: Krammer, K.; Lange-Bertalot, H. 1986, p. 348, pl. 151, Figure 6 (as Amphora coffeaeformis var. acutiuscula); Wah, T.T.; Wee, Y.C. 1988, Figures 11 and 12; Witkowski, A.; Lange-Bertalot, H.; Metzeltin, D. 2000 , p. 128 , pl. 161, Figures 10-13.

Size: $28 \mu \mathrm{m}$ long, $6 \mu \mathrm{m}$ broad, dorsal striae 14 in $10 \mu \mathrm{m}(n=1)$ (Figure 34q). 
Halamphora capitata (R. Hagelstein) I. Álvarez-Blanco and S. Blanco 2014

Ref. illus.: Hagelstein, R. 1938, pl. 3, Figure 7; Wachnicka, A.H.; Gaiser, E.E. 2007, p. 415, Figures 98 and 99 (both as Amphora bigibba var. capitata).

Size: $17 \mu \mathrm{m}$ long, $4 \mu \mathrm{m}$ wide, dorsal striae 24 in $10 \mu \mathrm{m}(n=1)$ (Figure 34i).

Halamphora coffeiformis (C.A. Agardh) C. Mereschkowsky 1903

Ref. illus.: Witkowski, A.; Lange-Bertalot, H.; Metzeltin, D. 2000, p. 133, pl. 161, Figures 21-25 (as Amphora coffeaeformis var. coffeaeformis).

Size: $34-36 \mu \mathrm{m}$ long, 5 to $6 \mu \mathrm{m}$ broad, dorsal striae 13 in $10 \mu \mathrm{m}$, ventral striae 14 to 15 in $10 \mu \mathrm{m}(n=2)$ (Figure 34o,p).

\section{Halamphora costata (W. Smith) Z. Levkov 2009}

Ref. illus.: Witkowski, A.; Lange-Bertalot, H.; Metzeltin, D. 2000, p. 134, pl. 169, Figure 9 (as Amphora costata); Levkov, Z. 2009, p. 181, pl. 92, Figure 14.

Size: $45-89 \mu \mathrm{m}$ long, $6-12 \mu \mathrm{m}$ broad, dorsal striae 7 to 8 in $10 \mu \mathrm{m}(n=6)$ (Figure $33 \mathrm{~m}-\mathrm{q})$.

\section{Halamphora cuneata (P.T. Cleve) Z. Levkov 2009}

Ref. illus.: Witkowski, A.; Lange-Bertalot, H.; Metzeltin, D. 2000, p. 135, pl. 167, Figures 20 and 21 (as Amphora cuneata); Levkov, Z. 2009, p. 182, pl. 105, Figures 1-6; pl. 243, Figures 5 and 6.

Size: $29-52 \mu \mathrm{m}$ long, 5 to $6 \mu \mathrm{m}$ broad, dorsal striae $12-14$ in $10 \mu \mathrm{m}(n=2)$ (Figure 33c-f).

Remark: The morphometrics of Figure $31 \mathrm{c}-\mathrm{f}$ coincide better with Amphora maletracta var. constricta, but lack the wide hyaline area separating striae bands on the dorsal margin.

\section{Halamphora exigua (W. Gregory) Z. Levkov 2009}

Ref. illus.: Witkowski, A.; Lange-Bertalot, H.; Metzeltin, D. 2000, p. 137, pl. 161, Figures 15-17 (as Amphora exigua).

Size: $44 \mu \mathrm{m}$ long, $6 \mu \mathrm{m}$ broad, dorsal striae 11 in $10 \mu \mathrm{m}(n=1)$ (Figure 35g).

\section{Halamphora wisei (M.M. Salah) I. Álvarez-Blanco and S. Blanco 2014}

Ref. illus.: Simonsen, R. 1962, p. 94, pl. 3, Figure 2; Witkowski, A.; Lange-Bertalot, H.; Metzeltin, D. 2000, p. 154, pl. 162, Figures 18 and 19 (both as Amphora wisei).

Size: $16 \mu \mathrm{m}$ long, $5 \mu \mathrm{m}$ broad, dorsal striae 14 in $10 \mu \mathrm{m}(n=2)$ (Figure $34 \mathrm{~m}, \mathrm{n}$ ).

Family: Berkeleyaceae D.G. Mann 1990

PARLIBELLUS E.J. Cox 1982

Parlibellus delognei (H. Van Heurck) E.J. Cox 1988

Ref. illus.: Hustedt, F. 1961-1966, p. 302, Figure 1422 (as Navicula grevillii); Witkowski, A.; Lange-Bertalot, H.; Metzeltin, D. 2000, p. 321, pl. 104, Figures 1-5.

Size: $26-34 \mu \mathrm{m}$ long, $8-12 \mu \mathrm{m}$ broad, striae 18-19 in $10 \mu \mathrm{m}(n=2)$ (Figure 30n-p).

\section{Parlibellus rhombicula (F. Hustedt) A. Witkowski 2000}

Ref. illus.: Hustedt, F. 1961-1966, p. 327, Figure 1422; Witkowski, A.; Lange-Bertalot, H.; Metzeltin, D. 2000, p. 325, pl. 103, Figure 3.

Size: 51-94 $\mu \mathrm{m}$ long, $11-19 \mu \mathrm{m}$ broad, striae 16-18 in $10 \mu \mathrm{m}(n=1)$ (Figure 30g,h,k-m).

\section{Parlibellus rhombicus (W. Gregory) E.J. Cox 1988*}

Ref. illus.: Cox, E.J. 1988, p. 25, Figures 17, 33-38.

Size: $73 \mu \mathrm{m}$ long, $12 \mu \mathrm{m}$ broad, striae $17-19$ in $10 \mu \mathrm{m}(n=1)$ (Figure 30i,j).

\section{Parlibellus weissflogii (A. Grunow) E.J. Cox 1988 *}

Ref. illus.: Cleve, P.T. 1878, p. 7; pl. 1, Figure 9 (as Brebissonia weissflogii)

Size: $52-66 \mu \mathrm{m}$ long, $22-31 \mu \mathrm{m}$ broad, striae $14-18$ in $10 \mu \mathrm{m}(n=2)$ (Figure 30a-f). 
Family: Diadesmidaceae D.G. Mann 1990

DIPLONEIS C.G. Ehrenberg 1844

Diploneis bombus (C.G. Ehrenberg) C.G. Ehrenberg 1853

Ref. illus.: Hustedt, F. 1931-1959, p. 704, Figure 1086a-c; Witkowski, A.; Lange-Bertalot, H.; Metzeltin, D. 2000, p. 183, pl. 86, Figures 1-29; pl. 2, Figures 1-3.

Size: $102 \mu \mathrm{m}$ long, $19 \mu \mathrm{m}$ broad, striae 8 in $10 \mu \mathrm{m}(n=1)$ (Figure 26a).

Diploneis chersonensis (A. Grunow) P.T. Cleve 1892

Ref. illus.: Schmidt, A.W.F. 1892, pl. 174, Figure 14; Witkowski, A.; Lange-Bertalot, H.; Metzeltin, D. 2000, p. 184, pl. 86, Figure 10.

Size: $92 \mu \mathrm{m}$ long, $31 \mu \mathrm{m}$ broad, striae 8 in $10 \mu \mathrm{m}(n=1)$ (Figure 26b,c).

Diploneis crabro (C.G. Ehrenberg) C.G. Ehrenberg 1854

Ref. illus.: Hustedt, F. 1931-1959, p. 616, Figure 1028; Witkowski, A.; Lange-Bertalot, H.; Metzeltin, D. 2000, p. 184, pl. 93, Figures 18-21.

Size: 39-51 $\mu \mathrm{m}$ long, 15-18 $\mu \mathrm{m}$ broad, striae 7-9 in $10 \mu \mathrm{m}(n=5)$ (Figure 26d-i).

\section{Diploneis incurvata (W. Gregory) P.T. Cleve 1894}

Ref. illus.: Hustedt, F. 1931-1959, p. 593, Figure 1012b-d; Witkowski, A.; Lange-Bertalot, H.; Metzeltin, D. 2000, p. 187, pl. 86, Figures 5-6; pl. 87, Figure 4.

Size: 25-35 $\mu \mathrm{m}$ long, 8.9-12 $\mu \mathrm{m}$ broad, striae 12 to 13 in $10 \mu \mathrm{m}(n=2)$ (Figure 27o,p).

\section{Diploneis gruendleri (A.W.F. Schmidt) P.T. Cleve 1894}

Ref. illus.: Hustedt, F. 1931-1959, p. 702, Figure 1084; Navarro, J.N. 1982, p. 34, pl. 22, Figure 5; Cremer, H.; Sangiorgi, F.; Wagner-Cremer, F.; McGee, V.; Lotter, A.F.; Visscher, H. 2007, p. 35, pl. 8, Figure 76. Size: $51 \mu \mathrm{m}$ long, $23 \mu \mathrm{m}$ broad, striae 9 in $10 \mu \mathrm{m}(n=1)$ (Figure 26j).

\section{Diploneis litoralis (A.S. Donkin) P.T. Cleve 1894}

Ref. illus.: Hendey, N.I. 1964, p. 226, pl. 32, Figure 9; Foged, N. 1984, p. 36, pl. 41 Figure 5.

Size: $36 \mu \mathrm{m}$ long, $12 \mu \mathrm{m}$ broad, striae 12 in $10 \mu \mathrm{m}(n=2)$ (Figure 27d).

\section{Diploneis litoralis var. clathrata (E. Østrup) P.T. Cleve 1896}

Ref. illus.: Hustedt, F. 1931-1959, p. 666, Figure 1062b,c; Witkowski, A.; Lange-Bertalot, H.; Metzeltin, D. 2000 , p. 188 , pl. 89 , Figures 5 and 7-13.

Size: $22-24 \mu \mathrm{m}$ long, $8-10 \mu \mathrm{m}$ broad, striae $19-21$ in $10 \mu \mathrm{m}(n=2)$ (Figure $27 \mathrm{k}, 1)$.

\section{Diploneis nitescens (W. Gregory) P.T. Cleve 1894}

Ref. illus.: Hustedt, F. 1931-1959, p. 640, Figure 1047; Witkowski, A.; Lange-Bertalot, H.; Metzeltin, D. 2000, p. 189, pl. 90, Figures 1-3; pl. 94, Figure 1.

Size: $49 \mu \mathrm{m}$ long, $18 \mu \mathrm{m}$ broad, striae 8 in $10 \mu \mathrm{m}(n=1)$ (Figure 27q,r).

\section{Diploneis novaeseelandiae (A.W.F.Schmidt) F. Hustedt 1937 *}

Ref. illus.: Hustedt, F. 1931-1959, p. 681, Figure 1073; Witkowski, A.; Lange-Bertalot, H.; Metzeltin, D. 2000, p. 190, pl. 94, Figure 11.

Size: $31 \mu \mathrm{m}$ long, $9 \mu \mathrm{m}$ broad, striae 11 in $10 \mu \mathrm{m}(n=1)$ (Figure 27s).

\section{Diploneis papula (A.W.F. Schmidt) P.T. Cleve 1894}

Ref. illus.: Hustedt, F. 1931-1959, p. 680, Figure 1071a-c; Witkowski, A.; Lange-Bertalot, H.; Metzeltin, D. 2000, p. 190, pls. 86, Figures 14 and 15; pl. 89, Figures 22-25.

Size: $21 \mu \mathrm{m}$ long, $11 \mu \mathrm{m}$ broad, striae 14 to 15 in $10 \mu \mathrm{m}(n=1)$ (Figure $27 \mathrm{n}$ ). 


\section{Diploneis smithii P.T. Cleve 1894}

Ref. illus.: Hustedt, F. 1931-1959, p. 647, Figure 1051; Witkowski, A.; Lange-Bertalot, H.; Metzeltin, D. 2000, p. 193, pl. 88, Figures 2-5; pl. 89, Figure 1.

Size: $35-53 \mu \mathrm{m}$ long, $19-27 \mu \mathrm{m}$ broad, striae 8 to 9 in $10 \mu \mathrm{m}(n=4)$ (Figure 26a-c).

\section{Diploneis suborbicularis (W. Gregory) P.T. Cleve 1894}

Ref. illus.: Lobban, C.S.; Schefter, M.; Jordan, R.W.; Arai, Y.; Sasaki, A.; Theriot, E.C.; Ashworth, M.; Ruck, E.C.; Pennesi, C. 2012, p. 291, pl. 46, Figures 2-4; Pennesi, C.; Caputo, A.; Lobban, C.S.; Poulin, M.; Totti, C.2017, Figures 51-57; Park, J.; Lobban, C.; Lee, K. 2018, p. 117, Figures 74 and 75.

Size: $36 \mu \mathrm{m}$ long, $22 \mu \mathrm{m}$ broad, striae 10 to 11 in $10 \mu \mathrm{m}(n=1)$ (Figure $27 \mathrm{~d}, \mathrm{e}$ ).

\section{Diploneis suspecta (A.W.F. Schmidt) N.I. Hendey 1958 *}

Ref. illus.: Schmidt, A.W.F. 1873, pl. 11, Figures 12, 13, 26, 27 (as Navicula suspecta).

Size: $30 \mu \mathrm{m}$ long, $9 \mu \mathrm{m}$ broad, striae 13 in $10 \mu \mathrm{m}(n=1)$ (Figure 27t).

\section{Diploneis vacillans var. renitens (A. W. F. Schmidt) P.T. Cleve 1894}

Ref. illus.: Hustedt, F. 1931-1959, p. 663, Figure 1060e-g; Witkowski, A.; Lange-Bertalot, H.; Metzeltin, D. 2000, p. 196, pl. 90, Figures 13 and 14.

Size: $30-38 \mu \mathrm{m}$ long, $10-13 \mu \mathrm{m}$ broad, striae $10-13$ in $10 \mu \mathrm{m}(n=6)$ (Figure $27 \mathrm{f}-\mathrm{j})$.

\section{Diploneis vacillans var. vacillans (A.W.F. Schmidt) P.T. Cleve 1894}

Ref. illus.: Hustedt, F. 1931-1959, p. 662, Figure 1060a-d; Witkowski, A.; Lange-Bertalot, H.; Metzeltin, D. 2000, p. 196, pl. 89, Figure 14; pl. 90, Figures 11 and 12; pl. 91, Figures 9 and 10.

Size: $20-35 \mu \mathrm{m}$ long, 7 to $8 \mu \mathrm{m}$ broad, striae 18 in $10 \mu \mathrm{m}(n=4)$ (Figure $27 \mathrm{~m}, \mathrm{u}, \mathrm{v})$.

CALONEIS P.T. Cleve 1894

\section{Caloneis elongata (A. Grunow) C.S. Boyer 1927}

Ref. illus.: Navarro, J.N. 1982, p. 33, pl. 21, Figure 8; Witkowski, A.; Lange-Bertalot, H.; Metzeltin, D. 2000, p. 164, pl. 152, Figure 10.

Size: $88 \mu \mathrm{m}$ long, $11 \mu \mathrm{m}$ broad, striae 17 in $10 \mu \mathrm{m}(n=1)$ (Figure 17z).

\section{Caloneis linearis (A. Grunow) C.S. Boyer 1927}

Ref. illus.: Hendey, N.I. 1964, p. 230. pl. 29, Figure 3; Witkowski, A.; Lange-Bertalot, H.; Metzeltin, D. 2000, p. 166, pl. 160, Figure 12.

Size: $50-55 \mu \mathrm{m}$ long, $6-8 \mu \mathrm{m}$ broad, striae 20 in $10 \mu \mathrm{m}(n=3)$ (Figure 17y).

Family: Naviculaceae F.T. Kützing 1844

NAVICULA J.B.G.M. Bory de Saint-Vincent 1822

Navicula arenaria var. rostellata $\mathrm{H}$. Lange-Bertalot 1985

Ref. illus.: Krammer, K.; Lange-Bertalot, H. 1985, p. 56, pl. 22, Figure 1; Witkowski, A.; Lange-Bertalot, H.; Metzeltin, D. 2000, p. 267, pl. 116, Figures 18-20; pl. 129, Figure 29.

Size: $59 \mu \mathrm{m}$ long, $12 \mu \mathrm{m}$ broad, striae 8 in $10 \mu \mathrm{m}(n=1)$ (Figure 31d).

\section{Navicula cancellata A.S. Donkin 1872}

Ref. illus.: Hendey, N.I. 1964, p. 203, pl. 30, Figures 18-20; Witkowski, A.; Lange-Bertalot, H.; Metzeltin, D. 2000, p. 271, pl. 138, Figures 1-3; pl. 144, Figures 1-7.

Size: $42-76 \mu \mathrm{m}$ long, $8 \mu \mathrm{m}$ broad, striae 6-9 in $10 \mu \mathrm{m}(n=3)$ (Figure 31e,f and Figure 32c,g).

\section{Navicula cluthensis W. Gregory 1854}

Ref. illus.: Hustedt, F. 1961-1966, p. 651, Figure 1653a-d; Witkowski, A.; Lange-Bertalot, H.; Metzeltin, D. 2000 , p. 273 , pl. 100, Figure 8. 
Size: $33 \mu \mathrm{m}$ long, $22 \mu \mathrm{m}$ broad, striae 14 in $10 \mu \mathrm{m}(n=1)$ (Figure 31j).

\section{Navicula diversistriata F. Hustedt 1955}

Ref. illus.: Hustedt, F. 1955, p. 28, pl. 9, Figures 6-9; Witkowski, A.; Lange-Bertalot, H.; Metzeltin, D. 2000, p. 275, pl. 136, Figures 1 and 2.

Size: 26-34 $\mu \mathrm{m}$ long, 11-17 $\mu \mathrm{m}$ broad, striae 10 to 11 in $10 \mu \mathrm{m}(n=2)$ (Figure 31m-p).

\section{Navicula johanrossi M.H. Giffen 1975}

Ref. illus.: Giffen, M.H. 1967, p. 268, Figures 63 and 64; Witkowski, A.; Lange-Bertalot, H.; Metzeltin, D. 2000, p. 284, pl. 129, Figure 18; pl. 137, Figures 1-10; pl. 147, Figure 7.

Size: $44 \mu \mathrm{m}$ long, $12 \mu \mathrm{m}$ broad, striae 12 in $10 \mu \mathrm{m}(n=1)$ (Figure 31g).

\section{Navicula longa var. longa (W. Gregory) J. Ralfs 1861}

Ref. illus.: Navarro, J.N. 1982, p. 45, pl. 28, Figure 5; Foged, N. 1984, p. 66, pl. 45, Figure 4.

Size: $105 \mu \mathrm{m}$ long, $18 \mu \mathrm{m}$ broad, striae 5 in $10 \mu \mathrm{m}(n=1)$ (Figure 32e,f).

\section{Navicula longa var. irregularis F. Hustedt 1955}

Ref. illus.: Hustedt, F. 1955, p. 28, pl. 9, Figure 1; Witkowski, A.; Lange-Bertalot, H.; Metzeltin, D. 2000, p. 288, pl. 135, Figures 8-12.

Size: $162 \mu \mathrm{m}$ long, 17 to $18 \mu \mathrm{m}$ broad, striae 5 to 6 in $10 \mu \mathrm{m}(n=2)$ (Figure 32a,b).

\section{Navicula lusoria M.H. Giffen 1975}

Ref. illus.: Giffen, M.H. 1975, p. 84, Figures 75-77; Witkowski, A.; Lange-Bertalot, H.; Metzeltin, D. 2000, p. 289, pl. 129, Figures 11-14.

Size: $21 \mu \mathrm{m}$ long, $8 \mu \mathrm{m}$ broad, striae 13 in $10 \mu \mathrm{m}(n=1)$ (Figure 31h,i).

\section{Navicula palpebralis var. angulosa (W. Gregory) H. Van Heurck $1885 *,+$}

Ref. illus.: Gregory, W. 1856, p. 42, pl. 10, Figure 22; Witkowski, A.; Lange-Bertalot, H.; Metzeltin, D. 2000, p. 294, pl. 140, Figures 4-7.

Size: $36-39 \mu \mathrm{m}$ long, $10 \mu \mathrm{m}$ broad, striae 10 to 11 in $10 \mu \mathrm{m}(n=3)$ (Figure 32h,i).

\section{Navicula pavillardi F. Hustedt 1939}

Ref. illus.: Hustedt, F. 1939, p. 635, Figures 86-90; Witkowski, A.; Lange-Bertalot, H.; Metzeltin, D. 2000, p. 295, pl. 116, Figures 5 and 6; pl. 130, Figure 18; pl. 131, Figures 2-6.

Size: $31-71 \mu \mathrm{m}$ long, $8-11 \mu \mathrm{m}$ broad, striae $8-11$ in $10 \mu \mathrm{m}(n=2)$ (Figure $31 \mathrm{c}, \mathrm{k}, \mathrm{l})$.

\section{Navicula pennata A.W.F. Schmidt 1876}

Ref. illus.: Hendey, N.I. 1964, p. 203, pl. 30, Figure 21; Witkowski, A.; Lange-Bertalot, H.; Metzeltin, D. 2000, p. 296, pl. 141, Figures 27 and 28.

Size: $62-77 \mu \mathrm{m}$ long, $12 \mu \mathrm{m}$ broad, striae 7 in $10 \mu \mathrm{m}(n=2)$ (Figures $31 \mathrm{~b}$ and $32 \mathrm{~d}$ ).

\section{Navicula transitans P.T. Cleve 1883}

Ref. illus.: Cleve, P.T. 1883, p. 467, pl. 36, Figure 31; Witkowski, A.; Lange-Bertalot, H.; Metzeltin, D. 2000, p. 309, pl. 127, Figures 6-8.

Size: $79 \mu \mathrm{m}$ long, $13 \mu \mathrm{m}$ broad, striae 8 in $10 \mu \mathrm{m}(n=1)$ (Figure 31a).

\section{Navicula valida var. minuta P.T. Cleve 1883 *}

Ref. illus.: Poulin, M.; Cardinal, A. 1982, p. 2840, Figure 29; Witkowski, A.; Lange-Bertalot, H.; Metzeltin, D. 2000, p. 312, pl. 128, Figures 14-16.

Size: $24 \mu \mathrm{m}$ long, $12 \mu \mathrm{m}$ broad, striae 9 in $10 \mu \mathrm{m}(n=1)$ (Figure 31q,r). 
SEMINAVIS D.G. Mann 1990

Seminavis barbarae A. Witkowski, H. Lange-Bertalot and D. Metzeltin 2000 *,

Ref. illus.: Witkowski, A.; Lange-Bertalot, H.; Metzeltin, D. 2000, p. 348, pl. 166, Figures 1-4 (as S. barbara).

Size: $45 \mu \mathrm{m}$ long, $4 \mu \mathrm{m}$ broad, dorsal striae 22 in $10 \mu \mathrm{m}$, ventral striae 20 in $10 \mu \mathrm{m}(n=1)$ (Figure 35m).

\section{Seminavis basilica D.B. Danielidis 2003}

Ref. illus.: Danielidis, D.B.; Mann, D.G. 2003, p. 22, Figures 1-19.

Size: $49-55 \mu \mathrm{m}$ long, $8 \mu \mathrm{m}$ broad, dorsal striae 26 to 27 in $10 \mu \mathrm{m}$, ventral striae 25 in $10 \mu \mathrm{m}(n=1)$ (Figure 35k).

\section{Seminavis macilenta (W. Gregory) D.B. Danielidis and D.G.Mann 2002}

Ref. illus.: Gregory, W. 1857, p. 510, Figure 65 (as Amphora macilenta); Danielidis, D.B.; Mann, D.G. 2002, p. 443, Figures 54-68.

Size: $68 \mu \mathrm{m}$ long, $8 \mu \mathrm{m}$ broad, dorsal striae 13 in $10 \mu \mathrm{m}$, ventral striae 13 in $10 \mu \mathrm{m}(n=1)$ (Figure 36e).

\section{Seminavis robusta D.B. Danielidis and D.G. Mann 2002}

Ref. illus.: Danielidis, D.B.; Mann, D.G. 2002, p. 440, Figures 39-53; Wachnicka, A.H.; Gaiser, E.E. 2007, p. 442, Figures 221-225.

Size: $52 \mu \mathrm{m}$ long, $8 \mu \mathrm{m}$ broad, dorsal striae 17 in $10 \mu \mathrm{m}$, ventral striae 16 in $10 \mu \mathrm{m}$ (Figures 35j and 36d).

\section{TRACHYNEIS P.T. Cleve 1894}

\section{Trachyneis aspera (C.G. Ehrenberg) P.T. Cleve 1894}

Ref. illus.: Hendey, N.I. 1964, p. 236, pl. 29, Figure 13; Witkowski, A.; Lange-Bertalot, H.; Metzeltin, D. 2000, p. 355 , pl. 159, Figures 1-6 and 9.

Size: $62-226 \mu \mathrm{m}$ long, $15-43 \mu \mathrm{m}$ broad, striae $9-13$ in $10 \mu \mathrm{m}(n=10)$ (Figure 29a-e).

\section{Trachyneis velata (A.W.F. Schmidt) P.T. Cleve 1894}

Ref. illus.: Hustedt, F. 1931-1959, p. 751, Figure 17; Witkowski, A.; Lange-Bertalot, H.; Metzeltin, D. 2000, p. 356, pl. 159, Figures 7 and 8.

Size: 52-75 $\mu \mathrm{m}$ long, 15-22 $\mu \mathrm{m}$ broad, striae 12-16 in $10 \mu \mathrm{m}(n=2)$ (Figure 29f,g).

Family: Plagiotropidaceae D.G.Mann 1990

PLAGIOTROPIS E. Pfitzer 1871

Plagiotropis australis (M. Peragallo) T.B.B. Paddock $1988^{*, \dagger}$

Ref. illus.: Peragallo, M. 1921, p. 59, pl. 3, Figure 13 (as Pseudoamphiprora australis); Paddock, T.B.B. 1988, p. 36, pl. 11, Figures 1-9.

Size: $226 \mu \mathrm{m}$ long, $23 \mu \mathrm{m}$ broad, striae 13 in $10 \mu \mathrm{m}(n=1)$ (Figure 34i).

Family: Pleurosigmataceae C. Mereschkowsky 1903

DONKINIA J. Ralfs 1861

Donkinia carinata (A.S. Donkin) J. Ralfs 1861

Ref. illus.: Foged, N. 1984, p. 48. pls. 40, Figures 1 and 2; pl. 41, Figure 1.

Size: $109-150 \mu \mathrm{m}$ long, $9-22 \mu \mathrm{m}$ broad, striae 20 in $10 \mu \mathrm{m}(n=2)$ (Figure 38j-1).

GYROSIGMA A.H. Hassall 1845

Gyrosigma balticum (C.G. Ehrenberg) L. Rabenhorst 1853

Ref. illus.: Hendey, N.I. 1964, p. 284, pl. 35, Figure 9; Foged, N. 1978, p. 73, pl. 21, Figure 1.

Size: $112 \mu \mathrm{m}$ long, $12 \mu \mathrm{m}$ broad, striae 14 in $10 \mu \mathrm{m}(n=1)$ (Figure $40 \mathrm{~b}$ ). 


\section{Gyrosigma parvulum F. Hustedt 1955 *}

Ref. illus.: Hustedt, F. 1955, p. 34, pl. 10, Figure 10.

Size: $61-93 \mu \mathrm{m}$ long, 5-7 $\mu \mathrm{m}$ broad, striae 20 in $10 \mu \mathrm{m}(n=4)$ (Figure 39e-g).

Remark: Our specimens are larger than those reported in [38], 45-50 $\mu \mathrm{m}$ long, $4 \mu \mathrm{m}$ broad, striae 40 in $10 \mu \mathrm{m}$.

\section{Gyrosigma peisonis (A. Grunow) F. Hustedt 1930}

Ref. illus.: Hustedt, F. 1955, p. 34, pl. 10, Figures 4 and 5; Navarro, J.N. 1982, p. 37, pl. 23, Figures 5 and 6.

Size: $104 \mu \mathrm{m}$ long, $11 \mu \mathrm{m}$ broad, striae 17 in $10 \mu \mathrm{m}(n=1)$ (Figure 40c).

\section{Gyrosigma reversum (W. Gregory) N.I. Hendey 1986 *}

Ref. illus.: Gregory, W. 1857, p. 530, pl. 14, Figures 105, 105b (as Pleurosigma reversum); Sterrenburg, F.A.S. 2000, p. 301, Figures 1-4.

Size: $92 \mu \mathrm{m}$ long, $6 \mu \mathrm{m}$ broad, striae 19 to 20 in $10 \mu \mathrm{m}(n=1)$ (Figure $40 \mathrm{~d}$ ).

Remark: Our specimen is smaller than the recorded by [39] 160-250 $\mu \mathrm{m}$ long, 12-15 wide.

\section{Gyrosigma tenuissimum var. hyperboreum (A. Grunow) P.T. Cleve 1894 *}

Ref. illus.: Grunow, A. 1880, p. 58, pl. 4, Figure 77 (as Pleurosigma tenuissimum var. hyperborea),

Size: $127 \mu \mathrm{m}$ long, $8 \mu \mathrm{m}$ broad, striae 19 in $10 \mu \mathrm{m}(n=1)$ (Figure 40a).

\section{PLEUROSIGMA W. Smith 1852}

\section{Pleurosigma formosum W. Smith 1852}

Ref. illus.: Moreno, J.L.; Licea, S.; Santoyo, H. 1996, p. 113, pl. 28, Figure 18; Sterrenburg, F.A.S.; Sar, E.A.; Sunesen, I. 2014, p. 2, Figures 1a-h and 3a.

Size: $235 \mu \mathrm{m}$ long, $29 \mu \mathrm{m}$ broad, transapical striae parallel 16 in $10 \mu \mathrm{m}$, oblique striae 12 in $10 \mu \mathrm{m}$ $(n=1)$ (Figure 41a).

\section{Pleurosigma cf. gracile F. Hustedt 1955 *}

Ref. illus.: Hustedt, F. 1955, p. 35, pl. 10, Figure 11.

Size: $120-128 \mu \mathrm{m}$ long, $16-18 \mu \mathrm{m}$ broad, transapical striae parallel 21 in $10 \mu \mathrm{m}$, oblique striae 20 in $10 \mu \mathrm{m}(n=2)$ (Figure 39d,e).

Remark: Description in [38] gives 30 transapical parallel striae in $10 \mu \mathrm{m}$ and 36 oblique striae $10 \mu \mathrm{m}$.

\section{Pleurosigma naviculaceum A. Brébisson 1854}

Ref. illus.: Shadbolt, G. 1853, p. 16, pl. 1, Figure 9; Foged, N. 1975, p. 50, pl. 17, Figure 4.

Size: $84 \mu \mathrm{m}$ long, $15 \mu \mathrm{m}$ broad, transapical striae parallel 19 to 20 in $10 \mu \mathrm{m}$, oblique striae 24 in $10 \mu \mathrm{m}$ $(n=2)$ (Figure 40e,f).

Remark: According to [40], P. diverse-striatum may be differentiated from P. inflatum because the latter shows an H-like depression on the ventral area of the valve, and by the angle of the raphe, $+10-13^{\circ}$, whilst in P. diverse-striatum the angle is $+17-21^{\circ}$. However, according to [27], P. naviculaceum is a synonym of $P$. inflatum, whilst in [28] P. naviculaceum is valid.

\section{Pleurosigma patagonicum var. paucistriatum E.A. Sar, F.A.S. Sterrenburg and I. Sunesen 2013}

Ref. illus.: Sar, E.A.; Sterrenburg, F.A.S.; Lavigne, A.S.; Sunesen, I. 2013, p. 35, Figures 15a-f, 16a-f and $17 \mathrm{a}-\mathrm{f}$.

Size: $125-146 \mu \mathrm{m}$ long; 14 to 15 broad $\mu \mathrm{m}$, transapical striae 20 to 21 in $10 \mu \mathrm{m}$, oblique striae 16 to 17 in $10 \mu \mathrm{m}(n=2)$ (Figure 39a,b). 
Pleurosigma rigidum W. Smith. 1853

Ref. illus.: Smith, W. 1853, p. 64, pl. 20, Figure 198; Sterrenburg, F.A.S. 2001, p. 124, Figures 7-10 and 19-22.

Size: $162 \mu \mathrm{m}$ long; 26 broad $\mu \mathrm{m}$, transapical striae 19 in $10 \mu \mathrm{m}$, oblique striae 17 in $10 \mu \mathrm{m}(n=4)$ (Figures $39 \mathrm{c}$ and $41 \mathrm{~b}-\mathrm{d}$ ).

\section{Pleurosigma subsalinum H. Peragallo 1891 *}

Ref. illus.: Peragallo, H. 1891: 24; pl. 8, Figures 16 and 17.

Size: $140 \mu \mathrm{m}$ long, 14 broad $\mu \mathrm{m}$, transapical striae 18 in $10 \mu \mathrm{m}$, oblique striae 15 in $10 \mu \mathrm{m}(n=1)$ (Figure 39f).

Family: Proschkiniaceae D.G.Mann 1990

PROSCHKINIA N.I. Karayeva 1978

Proschkinia complanata (A. Grunow) D.G. Mann 1990

Ref. illus.: Hustedt, F. 1955, p. 60, pl. 9, Figure 21; Witkowski, A.; Lange-Bertalot, H.; Metzeltin, D. 2000, p. 341, pl. 60, Figures 29-32; pl. 147, Figures 8-11.

Size: 37-66 $\mu \mathrm{m}$ long, 5-8 $\mu \mathrm{m}$ broad, striae 20-40 in $10 \mu \mathrm{m}(n=2)$ (Figure 38n,o).

Family: Scoliotropidaceae C. Mereschkowsky 1903

BIREMIS D.G. Mann and E.J. Cox 1990

Biremis lucens (F. Hustedt) Sabbe, Witkowski and Vyverman 1995

Ref. illus.: Simonsen, R. 1987, p. 174, pl. 275, Figures 27-29; Witkowski, A.; Lange-Bertalot, H.; Metzeltin, D. 2000, p. 159, pl. 155, Figures 9-15.

Size: $17 \mu \mathrm{m}$ long, $4 \mu \mathrm{m}$ broad, striae 11 in $10 \mu \mathrm{m}(n=1)$ (Figure 17s).

Family: Sellaphoraceae C. Mereschkowsky 1902

FALLACIA A.J. Stickle and D.G. Mann 1990

Fallacia litoricola (F. Hustedt) D. G. Mann 1990

Ref. illus.: Navarro, J.N.1982, p. 45, pl. 28, Figure 6 (as Navicula litoricola); Moreno, J.L.; Licea, S.; Santoyo, H. 1996, p. 72, pl. 21, Figure 3.

Size: $23-27 \mu \mathrm{m}$ long, $7-9 \mu \mathrm{m}$ broad, striae 18-20 in $10 \mu \mathrm{m}(n=3)$ (Figure 17v).

Fallacia vittata (P.T. Cleve) D. G. Mann 1990

Ref. illus.: Hustedt, F. 1961-1966, p. 371, Figure 1461 (Navicula vittata).

Size: $14-26 \mu \mathrm{m}$ long, $8-12 \mu \mathrm{m}$ broad, striae 17 to 18 in $10 \mu \mathrm{m}(n=2)$ (Figure $17 \mathrm{w}, \mathrm{x})$.

Order: Thalassiophysales D.G. Mann 1990

Family: Catenulaceae C. Mereschkowsky 1902

AMPHORA C.G. Ehrenberg 1844

Amphora americana A.H. Wachnicka and E.E. Gaiser 2007 *

Ref. illus.: Wachnicka, A.H.; Gaiser, E.E. 2007, p. 398, Figures 29-34.

Size: $48-52 \mu \mathrm{m}$ long, $4 \mu \mathrm{m}$ broad, dorsal striae 27 in $10 \mu \mathrm{m}(n=4)$ (Figure 35h).

\section{Amphora angustissima H. Heiden 1928}

Ref. illus.: Simonsen, R. 1992, pl. 69, Figures 1-8; Witkowski, A.; Lange-Bertalot, H.; Metzeltin, D. 2000, p. 128, pl. 156, Figure 6.

Size: $20-40 \mu \mathrm{m}$ long, $4-6 \mu \mathrm{m}$ broad, striae $12-16$ in $10 \mu \mathrm{m}(n=4)$ (Figure 33g-1).

\section{Amphora beaufortiana F. Hustedt 1955}

Ref. illus.: Hustedt, F. 1955, p. 38, pl. 14, Figures 1-5; Witkowski, A.; Lange-Bertalot, H.; Metzeltin, D. 2000, p. 131, pl. 168, Figures 10 and 11. 
Size: $31 \mu \mathrm{m}$ long, $5 \mu \mathrm{m}$ broad, striae 22 to 23 in $10 \mu \mathrm{m}(n=1)$ (Figure 351$)$.

\section{Amphora bigibba A. Grunow 1875}

Ref. illus.: Peragallo, H.; Peragallo, M. 1897-1908, p. 227, pl. 50, Figure 36; Foged, N. 1978, p. 32, pl. 35, Figure 4; pl. 36, Figure 13.

Size: $18-22 \mu \mathrm{m}$ long, 4 to $5 \mu \mathrm{m}$ broad, striae 19-21 in $10 \mu \mathrm{m}(n=4)$ (Figure $34 \mathrm{f}-\mathrm{h}, \mathrm{j}$ ).

Amphora bigibba var. interrupta (A. Grunow) P.T. Cleve 1895

Ref. illus.: Hustedt, F. 1955, p. 40, pl. 14, Figures 19-25; Witkowski, A.; Lange-Bertalot, H.; Metzeltin, D. 2000, p. 131, pl. 163, Figures 27-30.

Size: $50 \mu \mathrm{m}$ long, $12 \mu \mathrm{m}$ broad, striae 16 in $10 \mu \mathrm{m}(n=1)$ (Figure 34k,l).

\section{Amphora cingulata P.T. Cleve 1875}

Ref. illus.: Cleve, P.T. 1894-1895, p. 133, pl. 3, Figure 39; Foged, N. 1984, p. 17, pl. 52. Figure 6.

Size: 52-133 $\mu \mathrm{m}$ long, 9-22 $\mu \mathrm{m}$ broad $(n=2)$ (Figure 35a,b).

\section{Amphora crassa W. Gregory 1857}

Ref. illus.: Witkowski, A.; Lange-Bertalot, H.; Metzeltin, D. 2000, p. 134, pl. 169, Figures 5-7.

Size: $48 \mu \mathrm{m}$ long, $8 \mu \mathrm{m}$ broad, striae 6 in $10 \mu \mathrm{m}(n=1)$ (Figure 35o).

Amphora cf. cymbamphora B.J. Cholnoky 1960

Ref. illus.: Witkowski, A.; Lange-Bertalot, H.; Metzeltin, D. 2000, p. 136, pl. 164, Figures 26-28.

31-47 $\mu \mathrm{m}$ long, 5 to $6 \mu \mathrm{m}$ broad, striae 17 in $10 \mu \mathrm{m}(n=4)$ (Figure 35i).

Amphora graeffeana N.I. Hendey 1973

Ref. illus.: Witkowski, A.; Lange-Bertalot, H.; Metzeltin, D. 2000, p. 138, pl. 166, Figure 24; pl. 172, Figures 6-9.

Size: $52 \mu \mathrm{m}$ long, $11 \mu \mathrm{m}$ broad, striae 16 in $10 \mu \mathrm{m}(n=1)$ (Figure 35e,f).

\section{Amphora immarginata T. Nagumo 2003}

Ref. illus.: Witkowski, A.; Lange-Bertalot, H.; Metzeltin, D. 2000, pl. 162, Figure 20; Nagumo 2003, pp. 22 to 23, pls. 38-42; Wachnicka, A.H.; Gaiser, E.E. 2007, p. 429, Figures 161 and 162.

Size: $37-62 \mu \mathrm{m}$ long, $7-15 \mu \mathrm{m}$ broad, striae 13-15 in $10 \mu \mathrm{m}(n=4)$ (Figure 34b-e).

\section{Amphora laevissima W. Gregory 1857}

Ref. illus.: Witkowski, A.; Lange-Bertalot, H.; Metzeltin, D. 2000, p. 142, pl. 168, Figures 5-7.

Size: $42-46 \mu \mathrm{m}$ long, $6-8 \mu \mathrm{m}$ broad $(n=2)$ (Figure 35c,d).

\section{Amphora marina W. Smith 1857}

Ref. illus.: Witkowski, A.; Lange-Bertalot, H.; Metzeltin, D. 2000, p. 144, pls. 162, Figures 8-14; pl. 166, Figures 9-11.

Size: $37-62 \mu \mathrm{m}$ long, 7-15 $\mu \mathrm{m}$ broad, dorsal striae 13 in $10 \mu \mathrm{m}$, ventral striae 12 in $10 \mu \mathrm{m}(n=1)$ (Figure 34s,t).

\section{Amphora obtusa W. Gregory 1857}

Ref. illus.: Schoeman, F.R.; Archibald, R.E.M. 1987, p. 126, 127, Figures 1-12; Wachnicka, A.H.; Gaiser, E.E. 2007, p. 435, Figure 186.

Size: $42 \mu \mathrm{m}$ long, $7 \mu \mathrm{m}$ broad $(n=1)$ (Figure 35n).

\section{Amphora proteus W. Gregory 1857}

Ref. illus.: Witkowski, A.; Lange-Bertalot, H.; Metzeltin, D. 2000, p. 148, pls. 161, Figures 1 and 2; pl. 162, Figures 5 and 6. 
Size: $62-68 \mu \mathrm{m}$ long, 9 to $10 \mu \mathrm{m}$ broad, dorsal striae 13 in $10 \mu \mathrm{m}$, ventral striae 15 in $10 \mu \mathrm{m}(n=1)$ (Figure $34 \mathrm{u}, \mathrm{v}$ ).

\section{Amphora proteus var. contigua P.T. Cleve 1895}

Ref. illus.: Peragallo, H.; Peragallo, M. 1897-1908, pl. 44; Wachnicka, A.H.; Gaiser, E.E. 2007, p. 429, Figures 157-160.

Size: $30 \mu \mathrm{m}$ long, $5 \mu \mathrm{m}$ broad, dorsal striae 17 in $10 \mu \mathrm{m}$, ventral striae 15 in $10 \mu \mathrm{m}(n=1)$ (Figure 34r).

\section{Amphora studeri C. Janisch 1876}

Ref. illus.: Peragallo, H.; Peragallo, M. 1897-1908, p. 214, pl. 47, Figure 18; López-Fuerte, F.O.; Siqueiros Beltrones, D.A.; Jakes-Cota U.; Tripp-Valdéz, A. 2019, p. 108, Figure 3 d, e (both as A. formosa var. studeri).

Size: $68 \mu \mathrm{m}$ long, $9 \mu \mathrm{m}$ broad, dorsal striae 23 to 24 in $10 \mu \mathrm{m}$, ventral striae 23 in $10 \mu \mathrm{m}(n=1)$ (Figure 33a,b).

\section{Amphora spectabilis W. Gregory 1857}

Ref. illus.: Witkowski, A.; Lange-Bertalot, H.; Metzeltin, D. 2000, p. 150, pl. 166, Figure 8; pl. 167, Figures 25 and 26 .

Size: $88 \mu \mathrm{m}$ long, $16 \mu \mathrm{m}$ broad, dorsal striae 8 in $10 \mu \mathrm{m}$, ventral striae 15 in $10 \mu \mathrm{m}(n=1)$ (Figure 34a).

Family: Thalassiophysaceae D.G. Mann 1990

THALASSIOPHYSA P.S. Conger 1954

Thalassiophysa hyalina (R.K. Greville) T.B.B. Paddock and P.A. Sims 1981

Ref. illus.: Greville, R.K. 1865, p. 6, pl. 5, Figure 11 (as Amphiprora hyalina); Schmidt, A.W.F. 1876, pl. 40, Figures 2 and 3 (as Amphora insecta).

Size: $65 \mu \mathrm{m}$ long, $12 \mu \mathrm{m}$ broad $(n=1)$ (Figure $38 \mathrm{~m}$ ).

Order: Surirellales D.G. Mann 1990

Family: Auriculaceae Hendey, N.I. 1964

AURICULA F. Castracane 1873

Auricula flabelliformis M. Voigt 1960 *,

Ref. illus.: Ruck, E.C.; Kociolek, J.P. 2004, pl. 2, Figure 5; pl. 6, Figure 3; Park, J.S.; Lee, S.D.; Kang, S.E.; Lee, J.H. 2014, p. 238, Figures 3b,c, 4i; Lobban, C.S. 2015, p.3, Figures 10 and 12.

Size: 95-108 $\mu \mathrm{m}$ long, $62-69 \mu \mathrm{m}$ broad, $16-18$ striae in $10 \mu \mathrm{m}(n=2)$ (Figure 37a).

Auricula pulchra (R.K. Greville) P.T. Cleve $1894{ }^{*},+$

Ref. illus.: Cleve, P.T. 1894, p. 20, pl. 2, Figure 23.

Size: 73-83 $\mu \mathrm{m}$ long, 9-12 (45) $\mu \mathrm{m}$ broad, 22 striae in $10 \mu \mathrm{m}(n=3)$ (Figure 37b-g).

Family: Entomoneidaceae C.W. Reimer 1975

ENTOMONEIS C.G. Ehrenberg 1845

Entomoneis paludosa (W. Smith) C.W. Reimer 1975

Ref. illus.: Peragallo, H.; Peragallo, M. 1897-1908, pl. 38, Figures 12-15; Witkowski, A.; Lange-Bertalot, H.; Metzeltin, D. 2000, p. 199, pl. 109, Figures 26 and 27; pl. 173, Figure 8.

Size: $45-138 \mu \mathrm{m}$ long, $12-25 \mu \mathrm{m}$ broad, striae 19-23 in $10 \mu \mathrm{m}(n=6)$ (Figure 38a-i).

Family: Surirellaceae F.T. Kützing 1844

CAMPYLODISCUS C.G. Ehrenberg 1844

Campylodiscus bicostatus W. Smith 1854

Ref. illus.: Krammer, K.; Lange-Bertalot, H. 1988, p. 215, pl. 178, Figures 1-6; Al-Handal, A.Y.; Thomas, E.W.; Pennesi, C. 2018, p. 143, Figure 103. 
Size: $19 \mu \mathrm{m}$ long, $20 \mu \mathrm{m}$ broad, striae 12 in $10 \mu \mathrm{m}(n=1)$ (Figure 46f).

Remark: C. bicostatus is a freshwater species but is also found in marine water [41,42].

\section{Campylodiscus fastuosus C.G. Ehrenberg 1845}

Ref. illus.: Ruck, E.C.; Kociolek, J.P. 2004, p. 34, pls. 39, Figures 1-6; pl. 40, Figure 7; pl. 41, Figure 17; Lobban 2015, p. 3, Figures 13, 14, 16 and 18-20; Park, J.; Lobban, C.; Lee, K. 2018, p. 132, Figure 175.

Size: $38-45 \mu \mathrm{m}$ long, $35-42 \mu \mathrm{m}$ broad, costae 3 to 4 in $10 \mu \mathrm{m}$, striae 9-11 in $10 \mu \mathrm{m}(n=2)$ (Figure 46h,i).

\section{Campylodiscus neofastuosus E.C. Ruck and T. Nakov 2016}

Ref. illus.: Navarro, J.N. 1983, p. 402, Figures 69-71; Lobban, C.S.; Schefter, M.; Jordan, R.W.; Arai, Y.; Sasaki, A.; Theriot, E.C.; Ashworth, M.; Ruck, E.C.; Pennesi, C. 2012, p. 307, pl. 67, Figures 2 and 3; pl. 68, Figures 1-3 (both as Surirella fastuosa); Park, J.; Lobban, C.; Lee, K. 2018, p. 134, Figures 177-180. Size: 35-68 $\mu \mathrm{m}$ long, 25-50 $\mu \mathrm{m}$ broad, striae around central area 24-28 in $10 \mu \mathrm{m}(n=4)$ (Figure 46a-c).

\section{Campylodiscus ralfsii W. Smith 1853}

Ref. illus.: Foged, N. 1984, p. 27, pl. 60, Figure 5; Witkowski, A.; Lange-Bertalot, H.; Metzeltin, D. 2000, p. 413, pl. 214, Figure 16; Navarro, J.N.; Lobban, C.S. 2009, p. 150, Figures 128 and 129.

Size: $38-40 \mu \mathrm{m}$ long, $39 \mu \mathrm{m}$ broad, costae 9 in $10 \mu \mathrm{m}(n=2)$ (Figure 46j,k).

Campylodiscus scalaris (M.H. Giffen) C.S. Lobban and J.S. Park $2018 *,+$

Ref. illus.: Witkowski, A.; Lange-Bertalot, H.; Metzeltin, D. 2000, p. 416, pl. 215, Figures 4-6; Hein, M.K.; Winsborough, B.M.; Sullivan. 2008, pl. 64, Figure 4; Lobban, C.S.; Schefter, M. 2012, p. 308, pl. 68, Figures 4-6; pl. 69, Figures 1 and 2 (all as Surirella scalaris).

Size: $18 \mu \mathrm{m}$ long, $14 \mu \mathrm{m}$ broad $(n=1)$ (Figure 461,m).

\section{Campylodiscus simulans W. Gregory 1857}

Ref. illus.: Schmidt, A.W.F.; Schmitz, M.; Fricke, F.; Müller, O.; Heiden, H.; Hustedt, F. 1874-1959, pl. 17, Figures 12-14.

Diameter $21 \mu \mathrm{m}, 4$ costae in $10 \mu \mathrm{m}$ (margin), central striae 11 in $10 \mu \mathrm{m}$ (Figure 46g).

CORONIA (C.G. Ehrenberg) C.G. Ehrenberg 1912

Coronia ambigua (R.K. Greville) E.C. Ruck and M.D. Guiry 2016

Ref. illus.: Schmidt, A.W.F.; Schmitz, M.; Fricke, F.; Müller, O.; Heiden, H.; Hustedt, F. 1874-1959, pl. 18, Figures 23-26; Williams, D.M. 1988, pl. 26, Figures 1 and 2; Ruck, E.C.; Kociolek, J.P. 2004, pls. 36-38; Lobban, C.S.; Schefter, M. 2012, p. 305, pl. 63, Figures 5-7; pl. 64, Figure 1 (all as Campylodiscus ambiguus).

Diameter $55 \mu \mathrm{m}(n=2)$ (Figure 46d,e).

PSAMMODICTYON D.G. Mann 1990

Psammodictyon panduriforme (W. Gregory) D.G. Mann 1990

Ref. illus.: Witkowski, A.; Lange-Bertalot, H.; Metzeltin, D. 2000, p. 397, pl. 184, Figures 13 and 14; pl. 186, Figures 1-3 (as Nitzschia panduriformis); Lobban, C.S.; Schefter, M. 2012, p. 304, pl. 62, Figures 3 and 4. Size: 68-136 $\mu \mathrm{m}$ long, 28-34 $\mu \mathrm{m}$ broad, striae 14 to 15 in $10 \mu \mathrm{m}(n=7)$ (Figure 42a-d,k,l,o).

\section{Psammodictyon panduriforme var. continuum (A. Grunow) P. Snoeijis 1998}

Ref. illus.: Krammer. K. Lange-Bertalot, H. 1988, pl. 38, Figures 6 and 7; Witkowski, A.; Lange-Bertalot, H.; Metzeltin, D. 2000, p. 398, pl. 183, Figure 6 (both as Nitzschia panduriformis var. continua).

Size: $12 \mu \mathrm{m}$ long, $6 \mu \mathrm{m}$ broad, striae 19 in $10 \mu \mathrm{m}(n=1)$ (Figure 42i,j). 
Size: $41 \mu \mathrm{m}$ long, $19 \mu \mathrm{m}$ broad, fibulae 9 to 10 in $10 \mu \mathrm{m}$, striae $16-18$ in $10 \mu \mathrm{m}(n=1)$ (Figure $42 \mathrm{e}-\mathrm{g})$.

\section{Psammodictyon roridum (M.H. Giffen) D.G. Mann 1990}

Ref. illus.: Giffen, M.H. 1975, p. 90, Figures 103 and 104; Witkowski, A.; Lange-Bertalot, H.; Metzeltin, D. 2000, p. 403, pl. 184, Figures 9-12 (both as Nitzschia rorida).

Size: $49 \mu \mathrm{m}$ long, $12 \mu \mathrm{m}$ broad, fibulae 10 in $10 \mu \mathrm{m}$, striae 24 in $10 \mu \mathrm{m}(n=1)$ (Figure $42 \mathrm{~h}$ ).
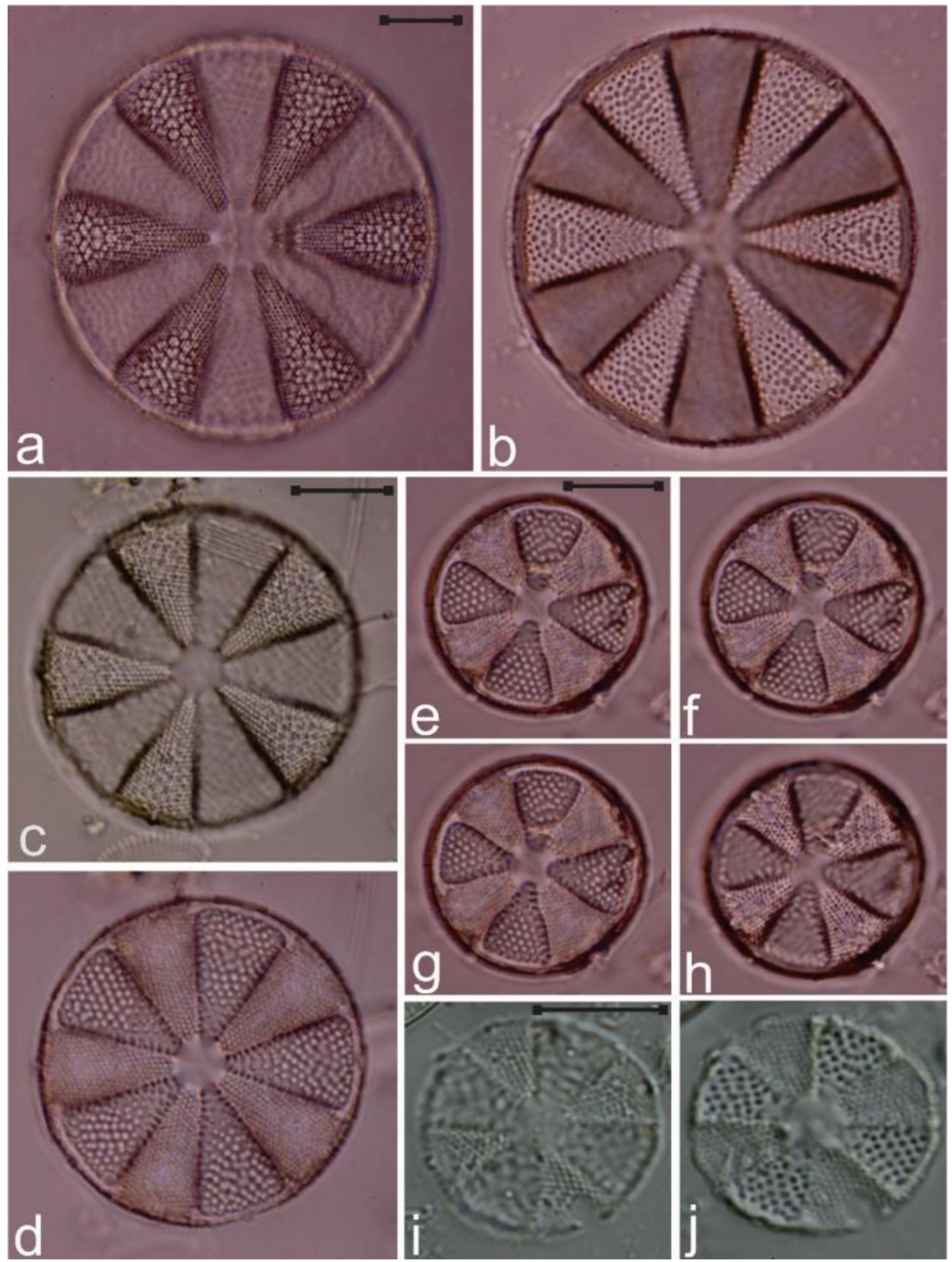

Figure 3. (a-d) Actinoptychus vulgaris in different focal planes; (e-j) A. minutus. Scale bar $=10 \mu \mathrm{m}$. 

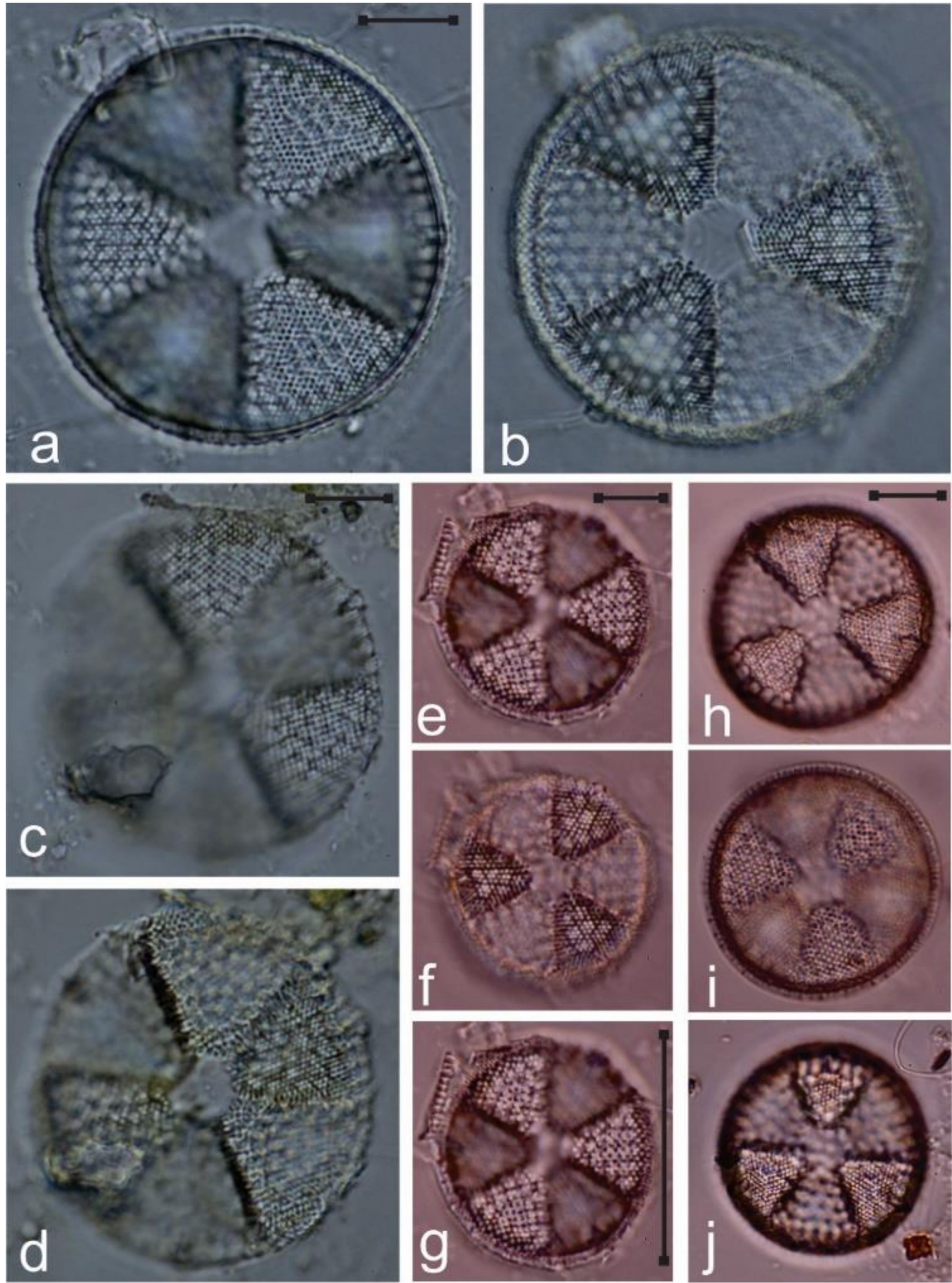

Figure 4. (a-j) Actinoptychus senarius in different focal planes and showing range in size. Scale bar $=10 \mu \mathrm{m}$. 

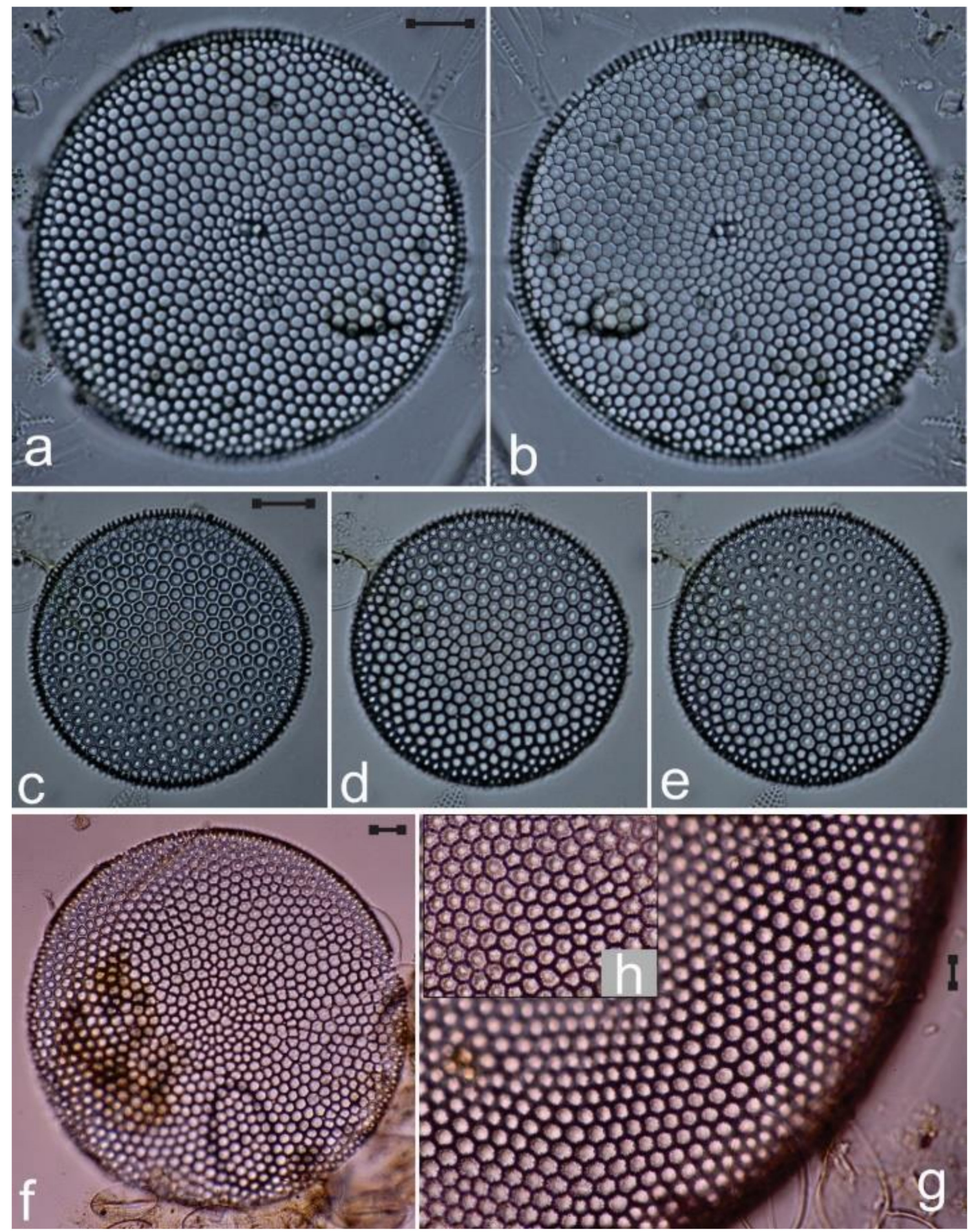

Figure 5. (a-f) Coscinodiscus radiatus valves in different focal views; $(\mathbf{g}, \mathbf{h})$ C. gigas, image $\mathrm{h}$ section of the valve (center) showing the arrangement of the areolae. Scale bar $=10 \mu \mathrm{m}$. 

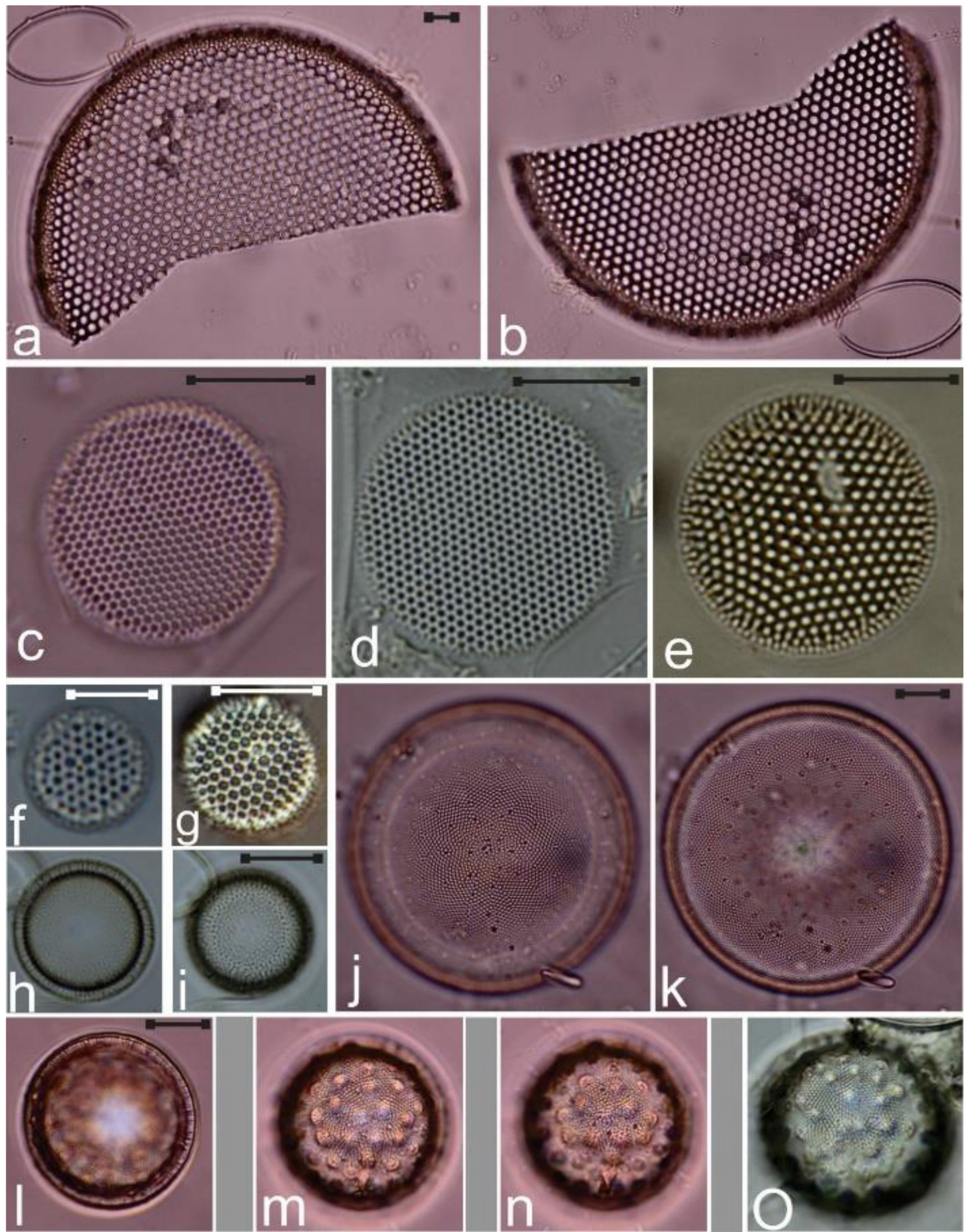

Figure 6. (a,b) Thalassiosira leptopus valve fragment in two focal planes; (c,d) T. lineata focus showing the rimoportulae; (e) T. eccentrica; $(\mathbf{f}, \mathbf{g})$ T. decipiens; $(\mathbf{h}, \mathbf{i})$ Melosira moniliformis var. octogona; $(\mathbf{j}, \mathbf{k})$ Coscinodiscus mesoleius; (1-o) Margaritum terebro, image 1 focus showing a side view of the rimoportulae near the margin, images $\mathbf{m}-\mathbf{o}$ focus showing rimoportulae papilliformis. Scale bar: $\mathbf{a}-\mathbf{e}, \mathbf{h}-\boldsymbol{n}=10 \mu \mathrm{m} ; \mathbf{f}=5 \mu \mathrm{m}$. 

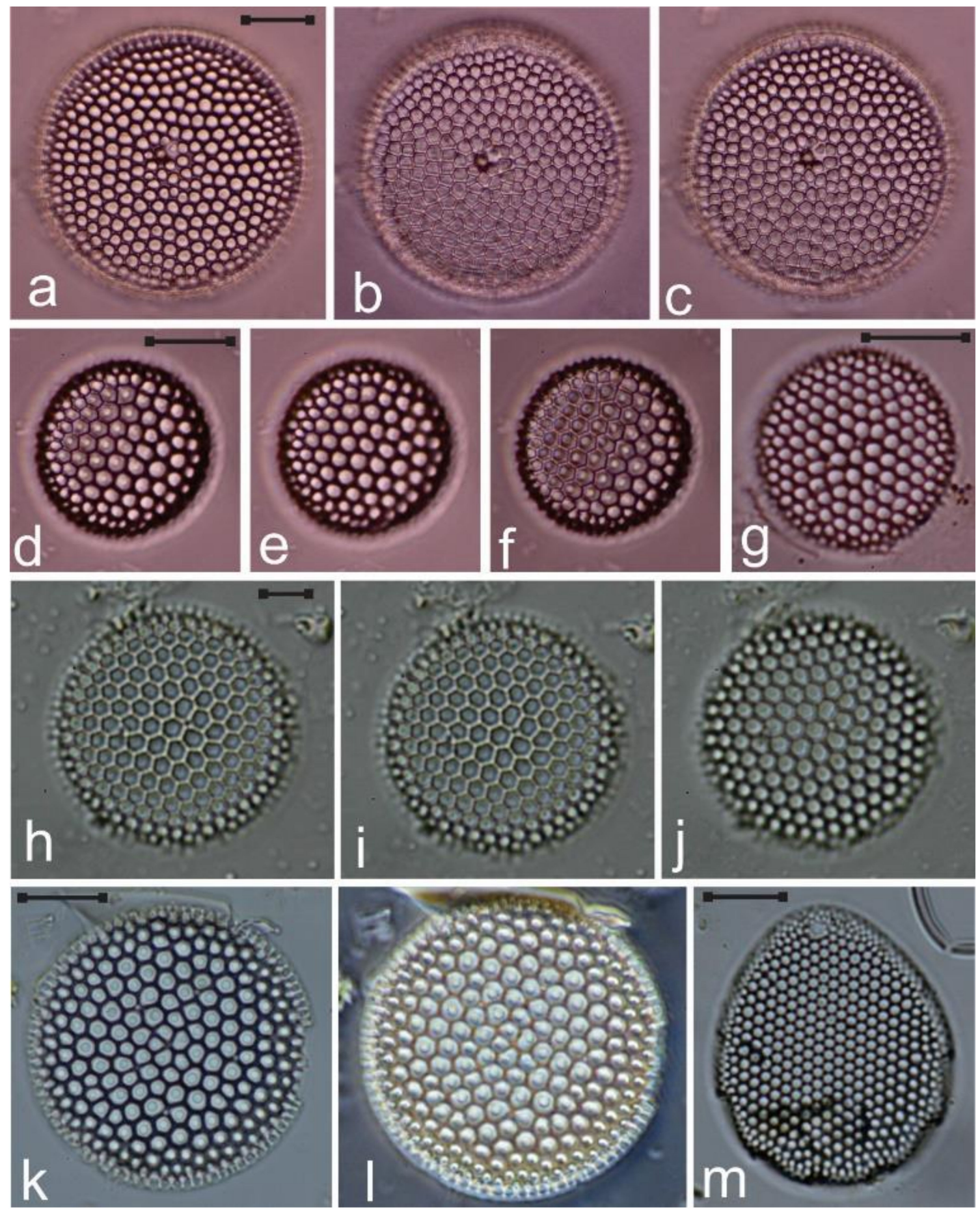

Figure 7. (a-g) Azpeitia nodulifera in different focal planes and showing range in size; $(\mathbf{h}-\mathbf{j})$ Thalassiosira nanolineata; $(\mathbf{k}, \mathbf{l})$ Coscinodiscus radiatus in different focal planes; $(\mathbf{m})$ Roperia tesselata. Scale bar $=10 \mu \mathrm{m}$. 

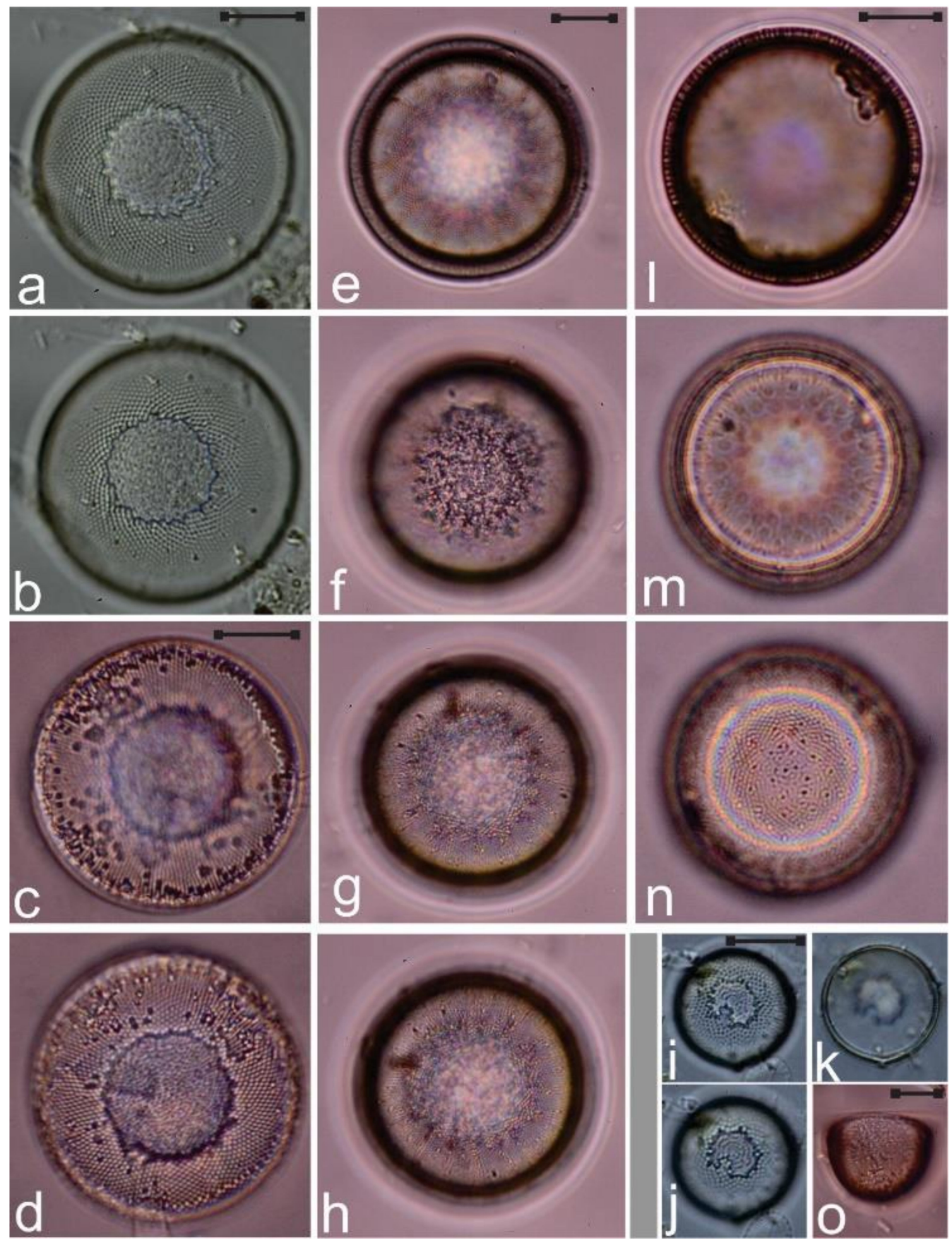

Figure 8. (a-k) Podosira stelligera in different focal planes and showing range in size; (1-n) P. variegata in different focal planes; (o) P. montagnei valve in girdle view. Scale bar $=10 \mu \mathrm{m}$. 

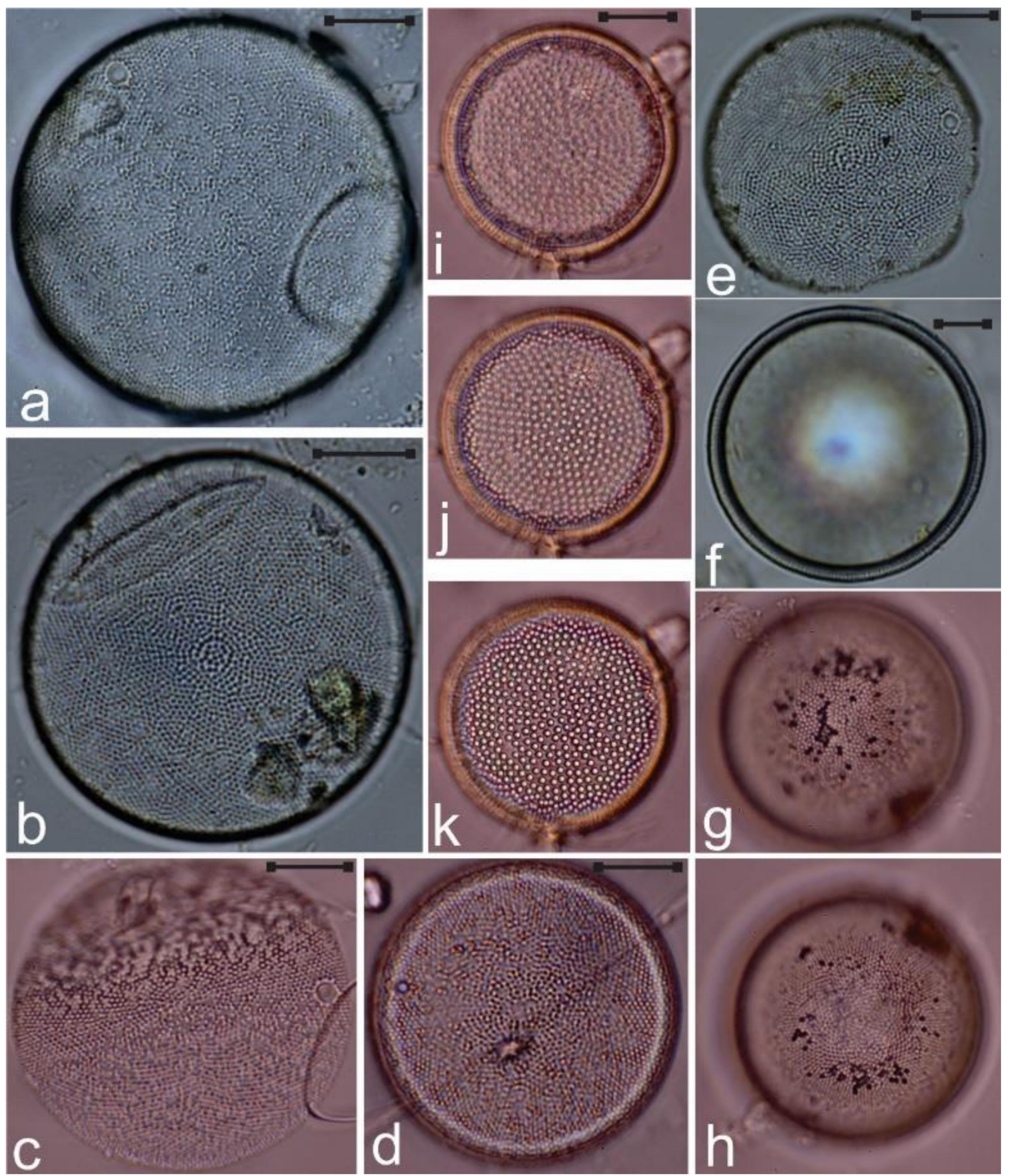

Figure 9. (a-e) Actinocyclus subtilis in different focal planes; (f-h) A. tenuissimus; (i-k) A. ochotensis. Scale bar $=10 \mu \mathrm{m}$. 

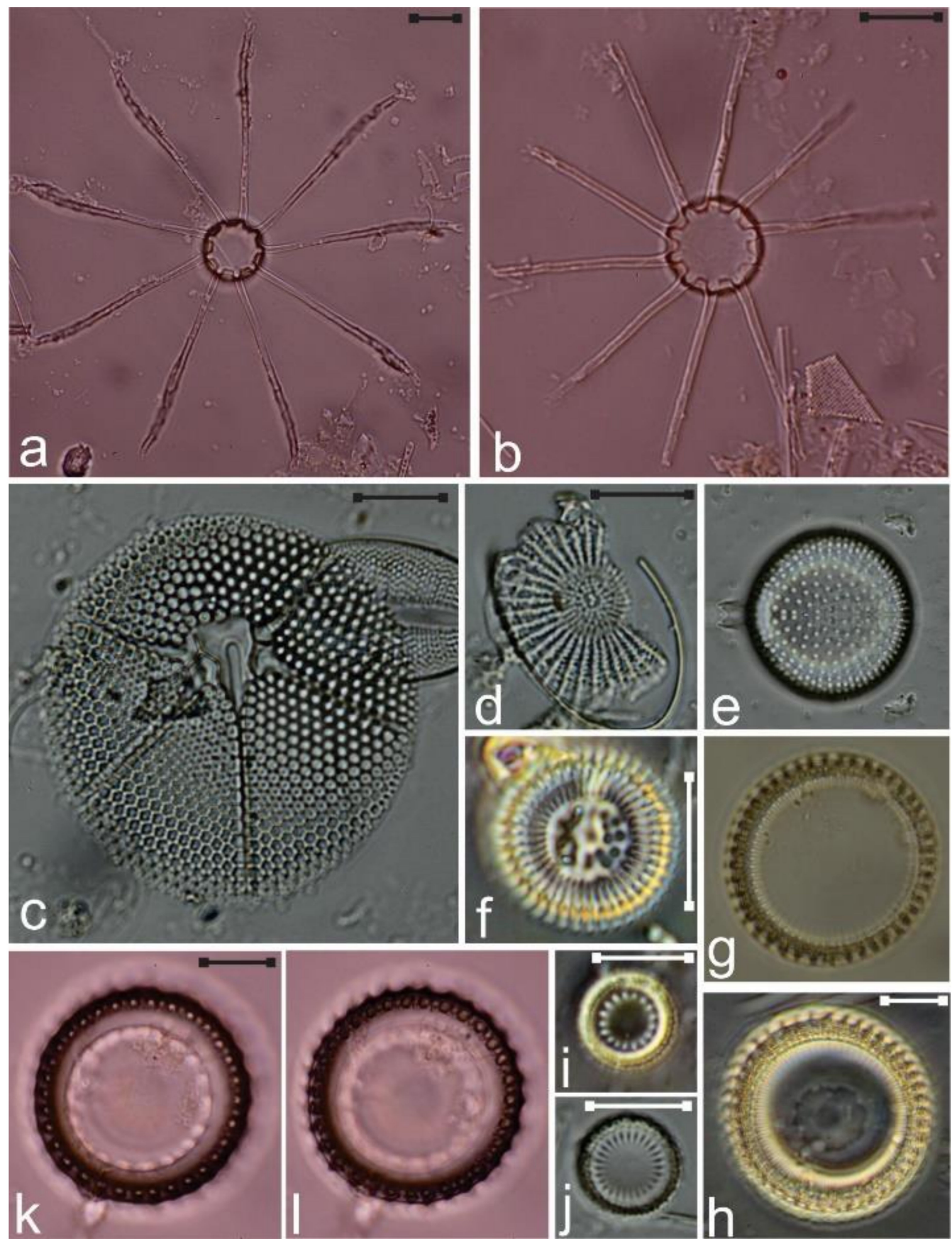

Figure 10. (a,b) Bacteriastrum hyalinum; (c) Asteromphalus arachne; (d) Cyclostephanos sp.; (e) Psammodiscus nitidus; (f) Cyclotella striata; (g-1) Paralia sulcata. Scale bar $=10 \mu \mathrm{m}$. 

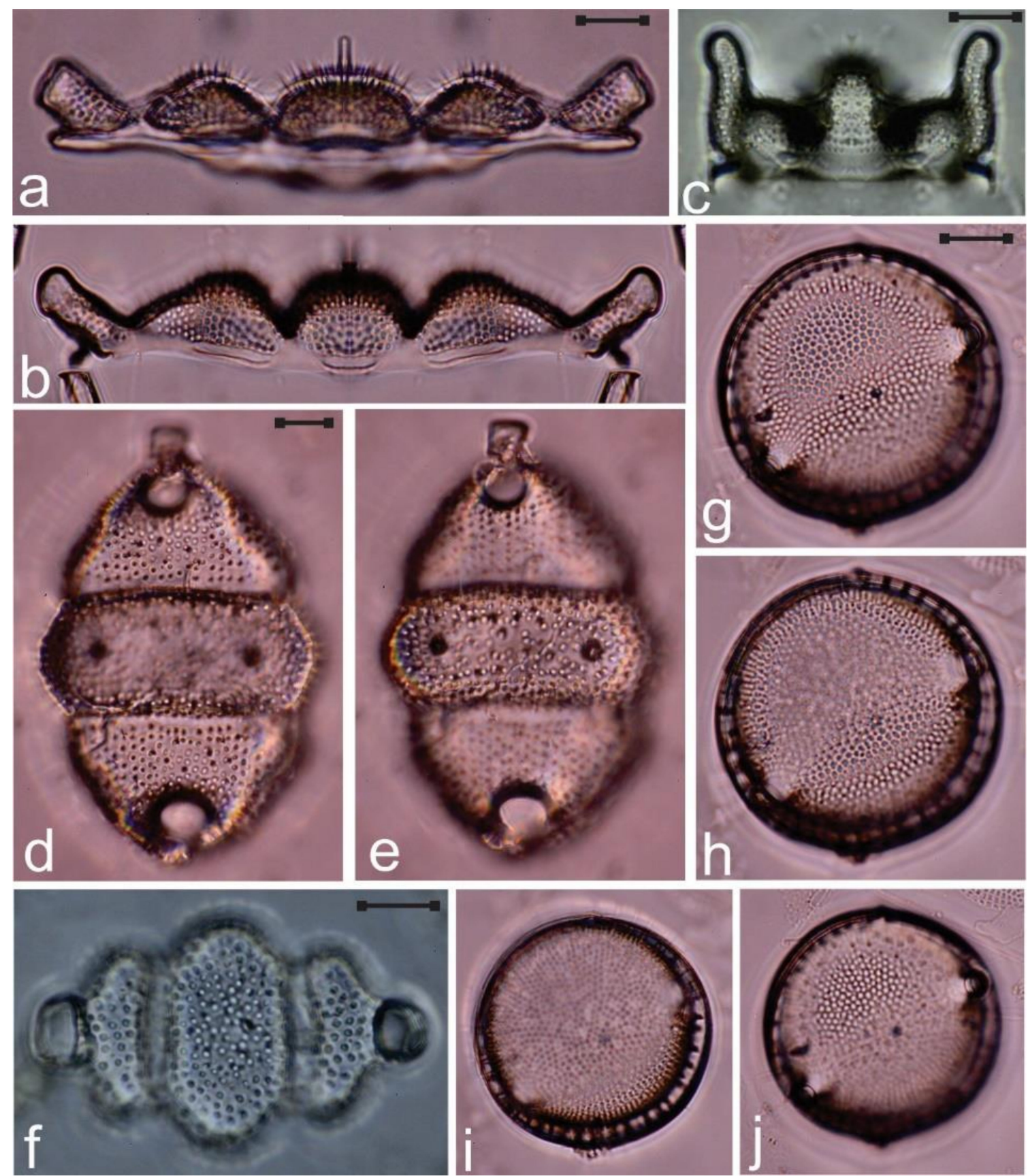

Figure 11. $(\mathbf{a}-\mathbf{c}, \mathbf{f})$ Biddulphia tridens, images a-c girdle view, image $\mathbf{f}$ valve view; $(\mathbf{d}, \mathbf{e})$ B. tridentata valve at two focal planes; $(\mathbf{g}-\mathbf{j})$ Ralfsiella smithii showing different focal planes. Scale bar $=10 \mu \mathrm{m}$. 

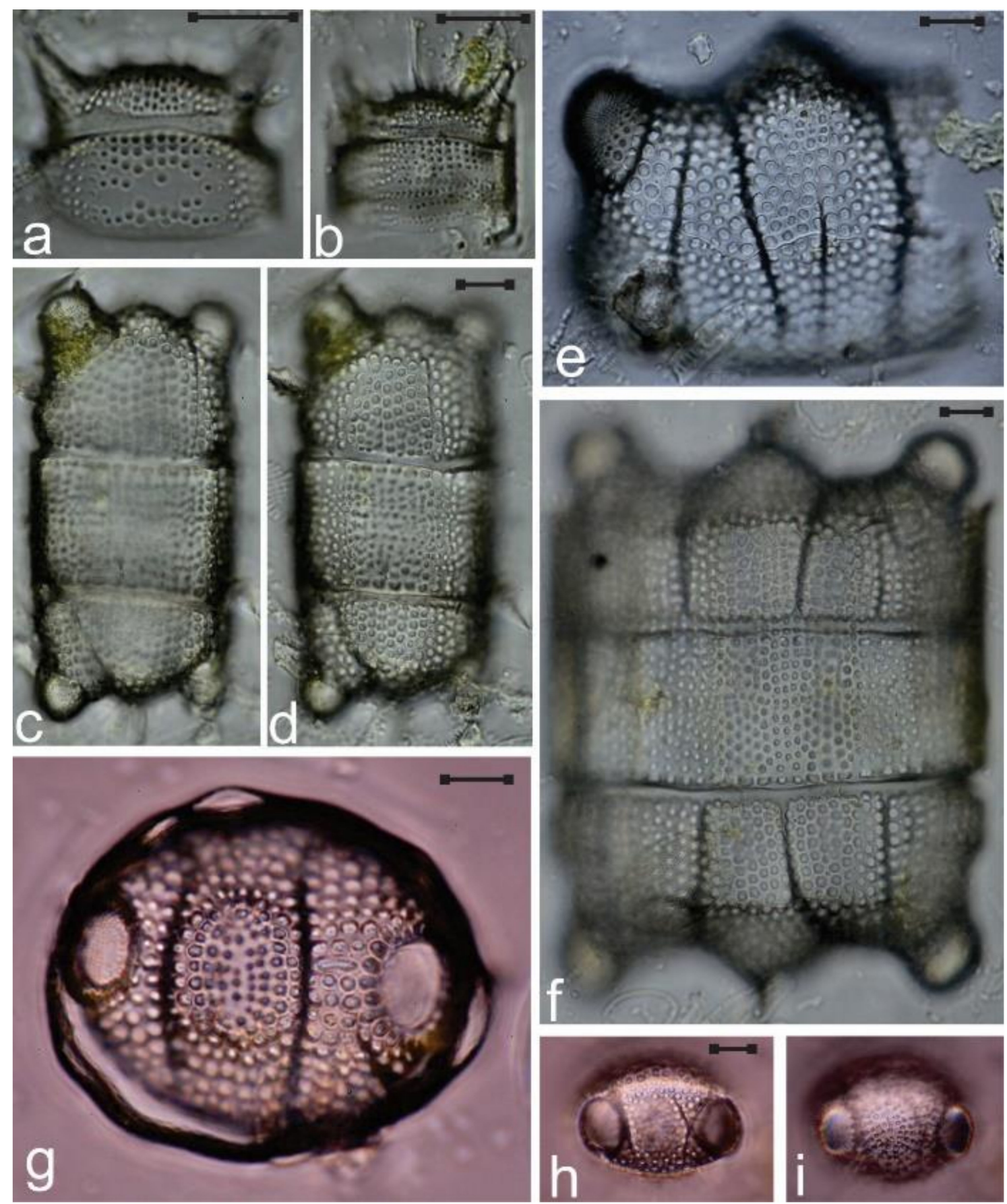

Figure 12. (a,b) Odontella rostrata; (c-i) Biddulphia biddulphiana images $\mathbf{c}, \mathbf{d}, \mathbf{f}$ frustule in girdle view showing the cingulum with full set of girdle bands. Scale bar $=10 \mu \mathrm{m}$. 

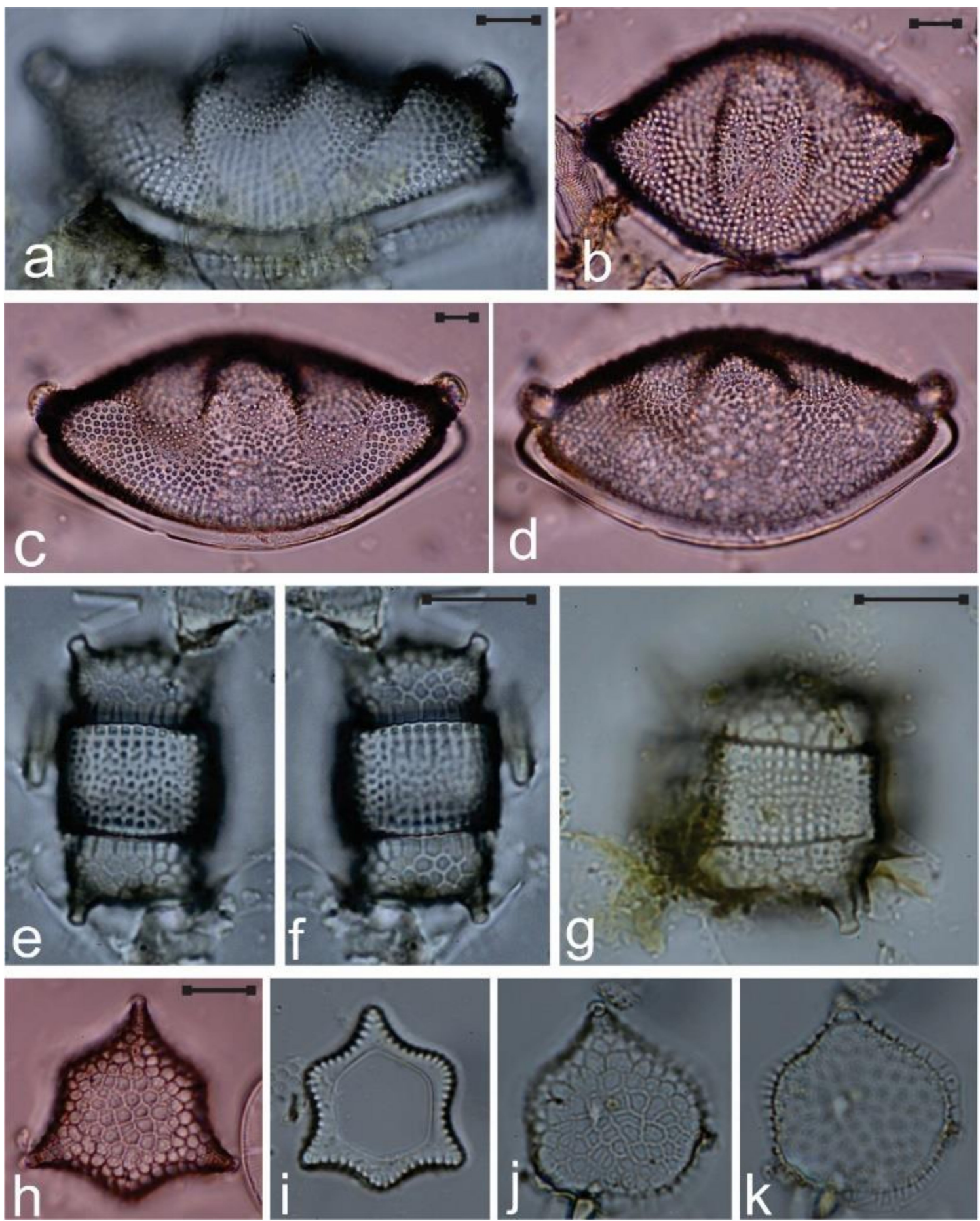

Figure 13. (a-d) Odontella obtusa; (e-k) Pseudictyota dubia, images $\mathbf{e}-\mathbf{g}$ frustule in girdle view showing the cingulum, images $\mathbf{h}-\mathbf{k}$ valve view at four focal planes. Scale bar $=10 \mu \mathrm{m}$. 

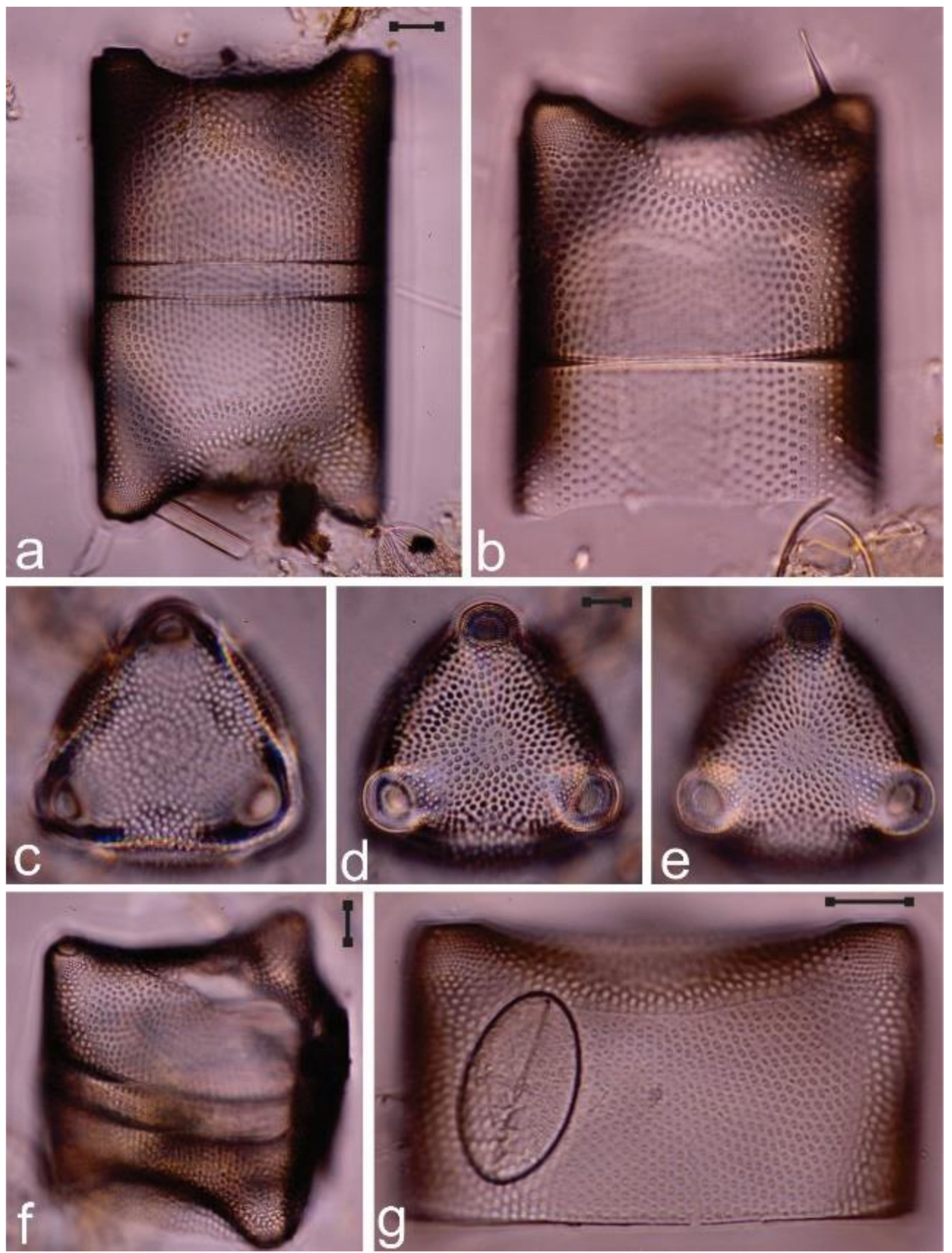

Figure 14. (a-g) Lampriscus shadboltianum, images $\mathbf{a}, \mathbf{b}, \mathbf{f}, \mathbf{g}$ frustule in girdle view showing the cingulum, image $\mathbf{b}$ valve exterior, short elevations with ocelli at apices and submarginal spine. Scale bar $=10 \mu \mathrm{m}$. 

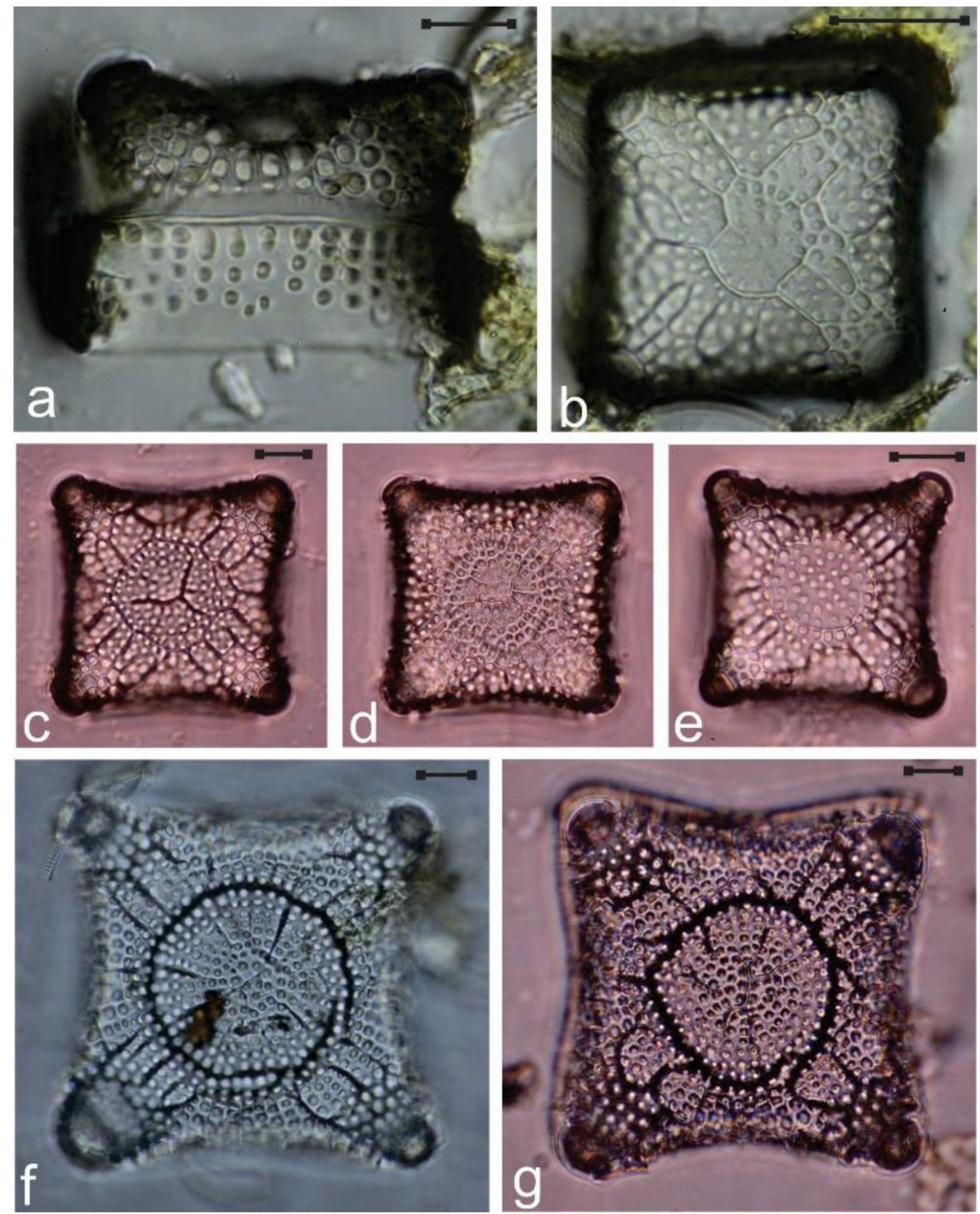

Figure 15. (a,b) Amphitetras antediluviana mantle view of valve with barely raised elevations; $(\mathbf{c}-\mathbf{g})$ Triceratium balearicum fo. biquadrate valve with four slightly raised elevations. Scale bar $=$ $10 \mu \mathrm{m}$. 

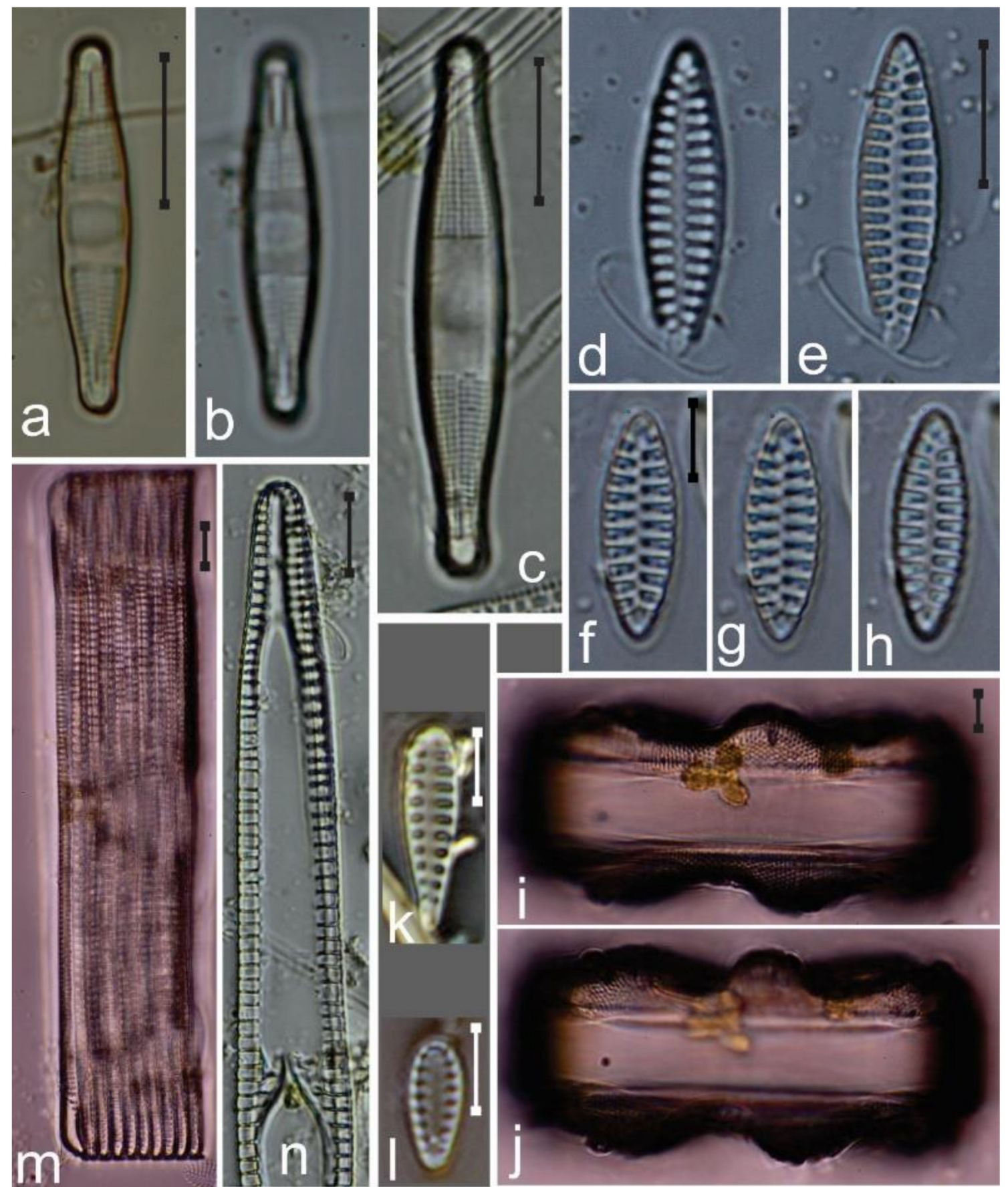

Figure 16. (a-c) Talaroneis sp.; (d-h) Opephora pacifica; (i,j) Aulacodiscus sp. frustule in girdle view showing the cingulum; (k) Gedaniella mutabilis; (1) Staurosirella guenter-grassii; (m,n) Rhabdonema adriaticum, image $m$ girdle view showing the bands. Scale bar: $\mathbf{a}-\mathbf{j}, \mathbf{m}, \mathbf{n}=10 \mu \mathrm{m} ; \mathbf{k}, \mathbf{l}=5 \mu \mathrm{m}$. 

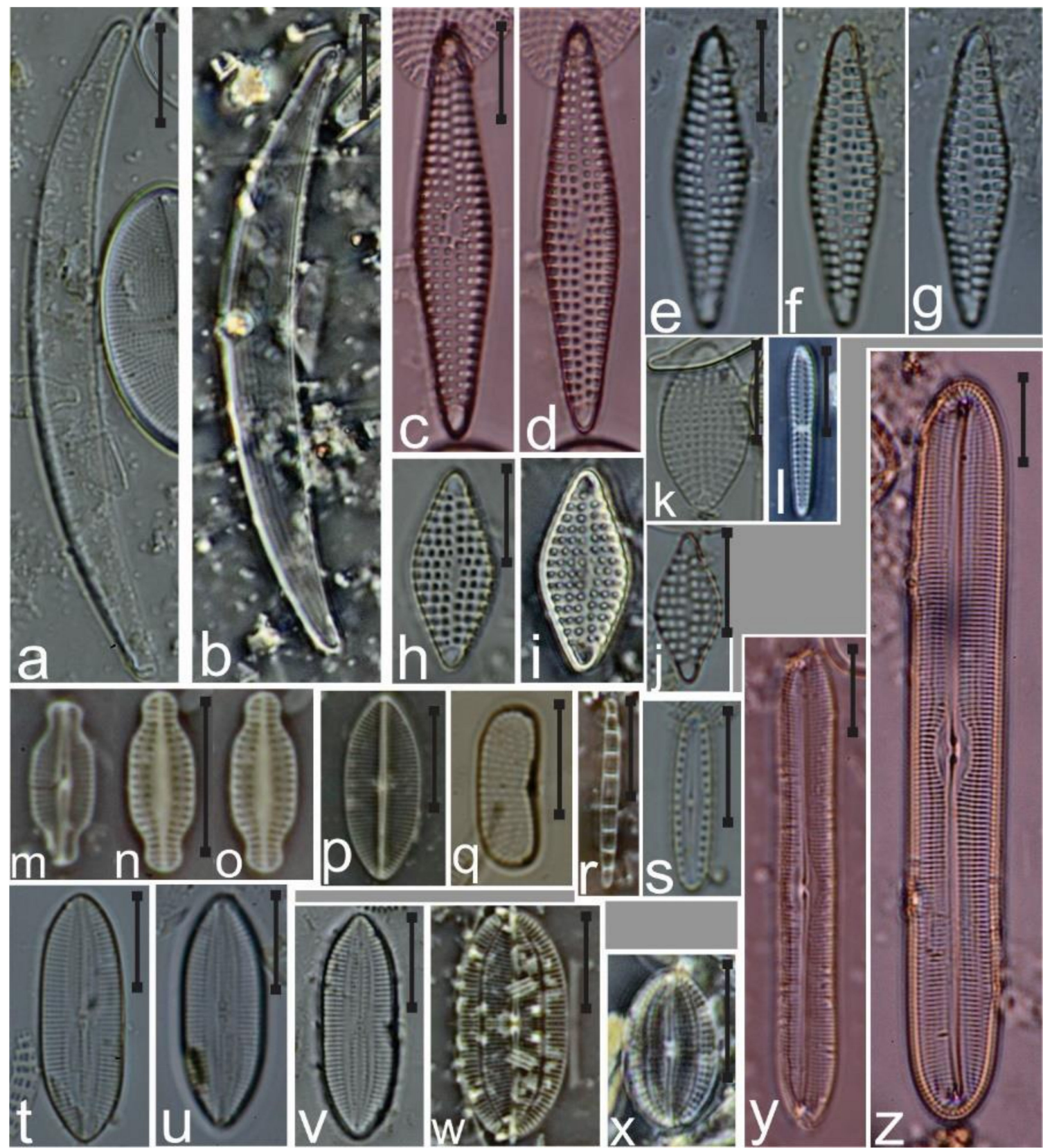

Figure 17. (a,b) Falcula media; (c,d) Dimeregramma fulvum; (e-j) D. minor; (k) Delphineis minutissima; (l) Gomphoseptatum aestuarii; $(\mathbf{m}-\mathbf{o})$ Karayevia amoena, image $\mathbf{m}$ raphe valve, images $\mathbf{n}$ and $\mathbf{o}$ rapheless valve; (p) Astartiella bahusiensis; (q) Colliculoamphora reichardtiana; (r) Nagumoea vallus; (s) Biremis lucens; $(\mathbf{t}-\mathbf{v})$ Fallacia litoricola $;(\mathbf{w}, \mathbf{x})$ F. vittata; $(\mathbf{y})$ Caloneis linearis; $(\mathbf{z})$ C. elongata. Scale bar $=10 \mu \mathrm{m}$. 


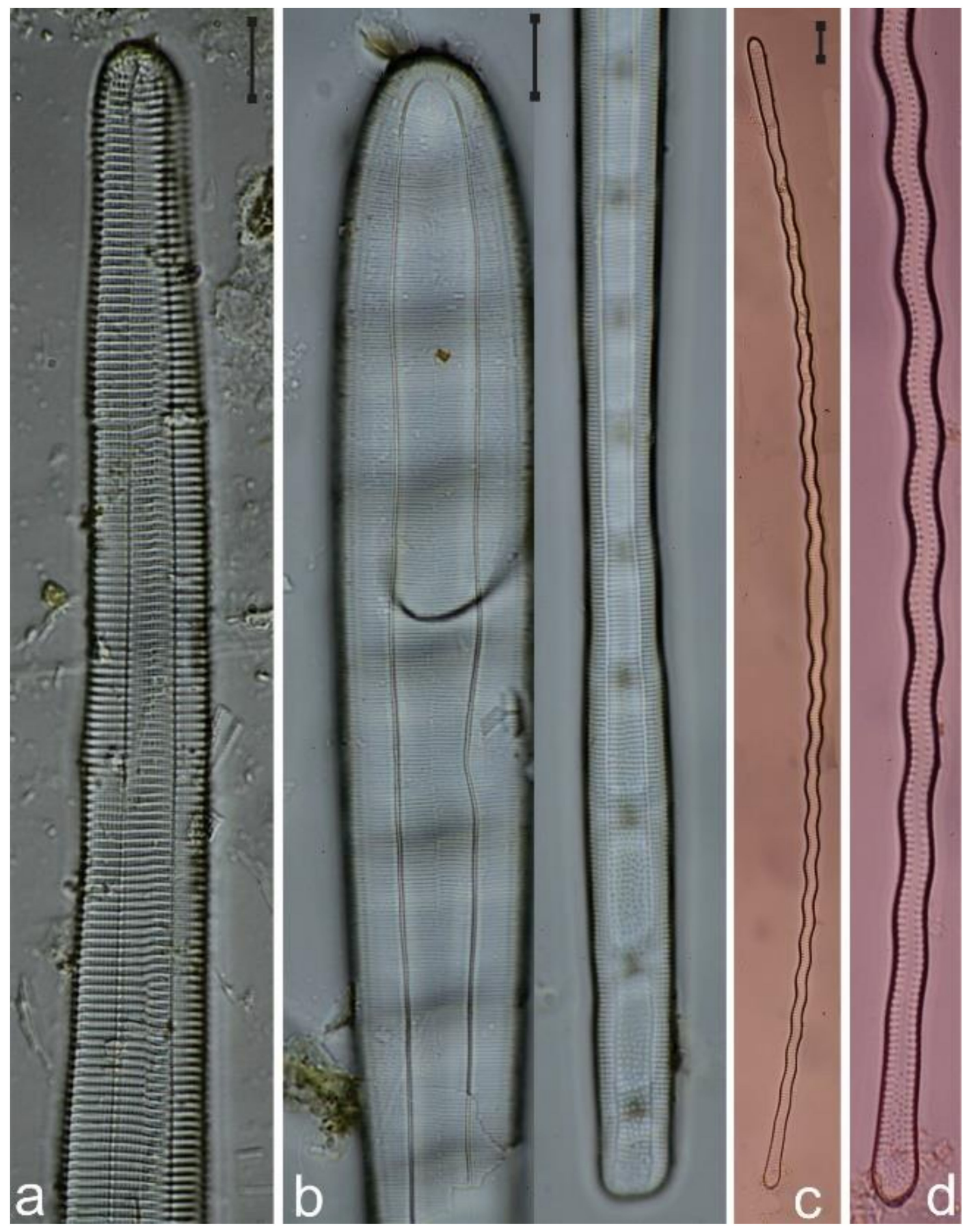

Figure 18. (a) Ardissonea formosa; (b) Climacosphenia elongata; (c,d) Toxarium undulatum. Scale bar $=10 \mu \mathrm{m}$. 


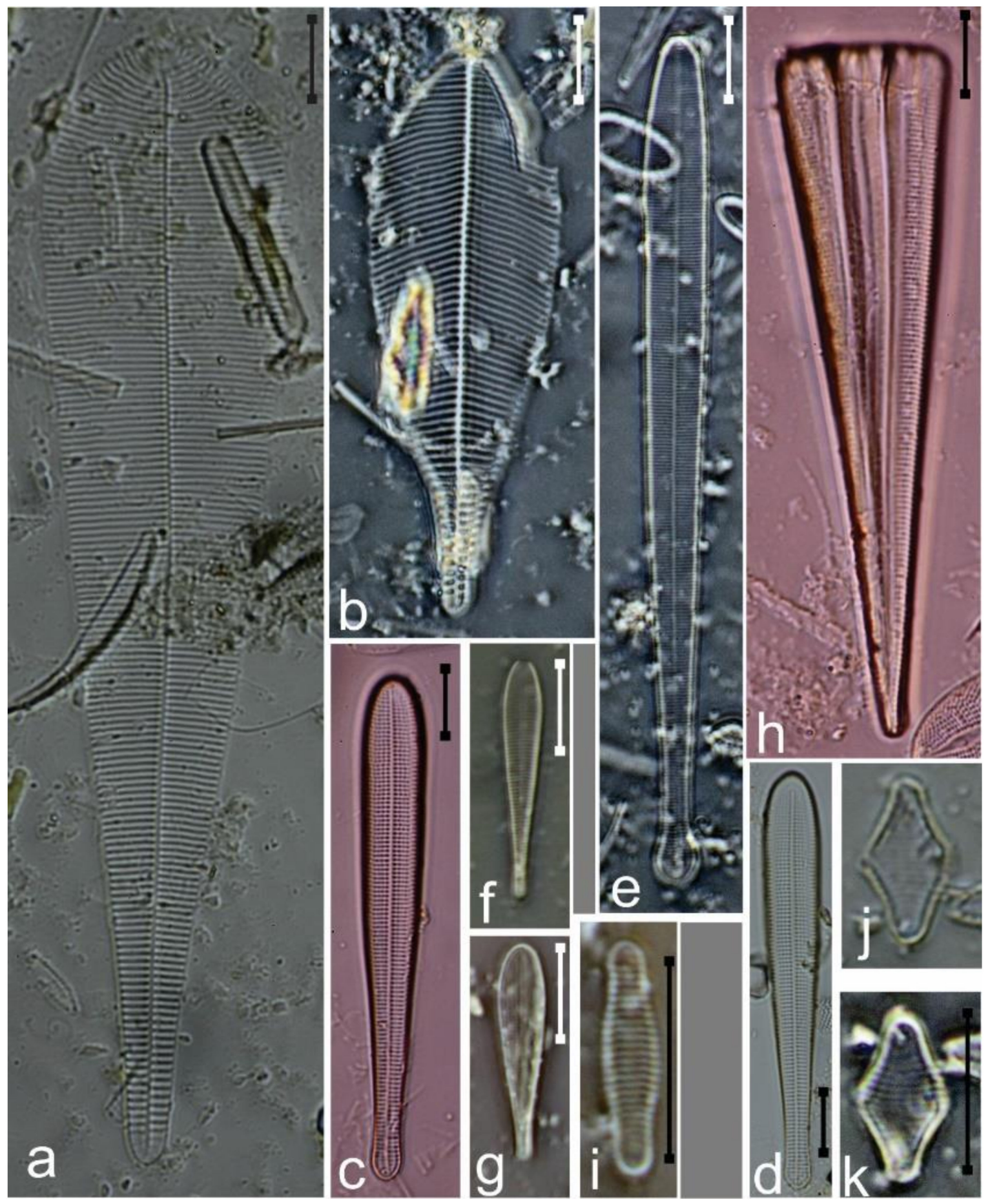

Figure 19. (a,b) Licmophora ehrenbergii; (c,d) L. paradoxa; (e) L. pfannkucheae; (f,g) L. gracilis var. anglica; (h) L. abbreviata frustule in girdle view; (i-k) Hyalosira tropicalis. Scale bar $=10 \mu \mathrm{m}$. 


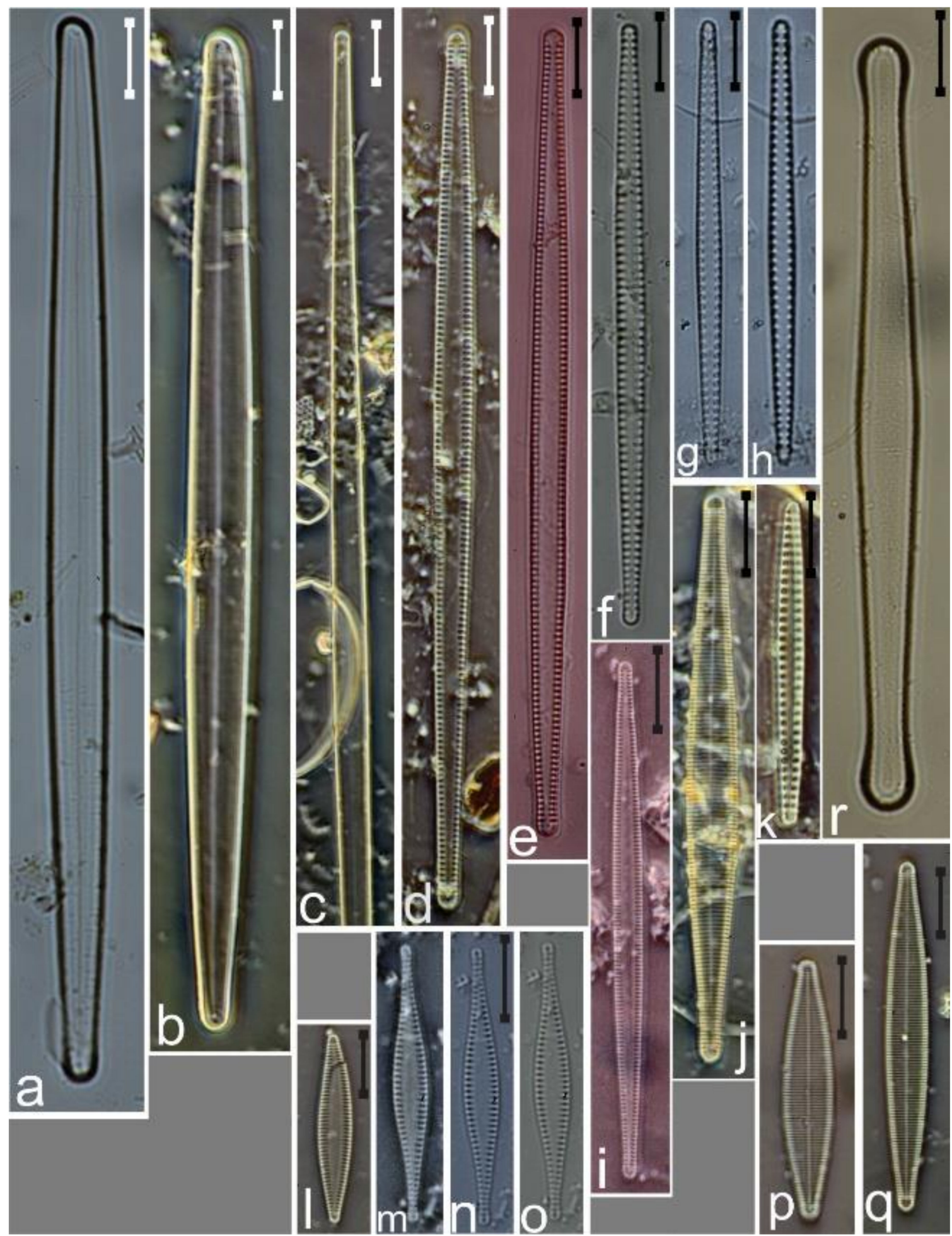

Figure 20. (a,b) Synedra gaillonii var. macilenta cf.; (c) Hyalosynedra laevigata; (d-f) Tabularia tabulata; $(\mathbf{g}, \mathbf{h}, \mathbf{k})$ T. investiens; (i) T. parva; (j,p,q) Fragilaria barbatula; (1) Tabularia fasciculata; (m-o) Synedra tabulata var. rostrata; (r) Grammatophora macilenta. Scale bar $=10 \mu \mathrm{m}$. 


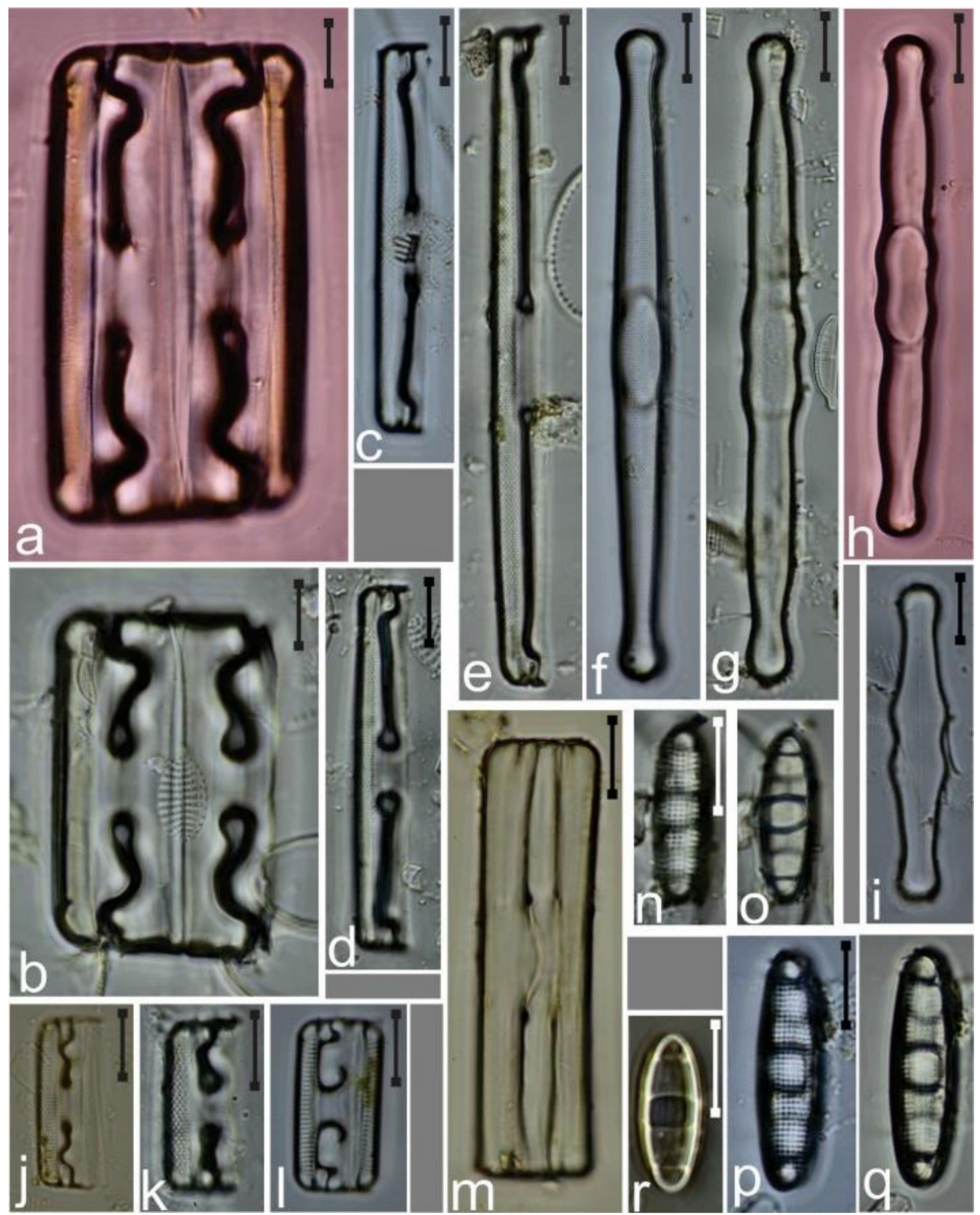

Figure 21. (a,b) Grammatophora macilenta; $(\mathbf{c}-\mathbf{f}, \mathbf{j})$ G. oceanica; (k) G. marina; $(\mathbf{g}-\mathbf{i}, \mathbf{m})$ G. undulata var. gallopagensis; $(\mathbf{l}, \mathbf{n}-\mathbf{r})$ G. hamulifera. Scale bar $=10 \mu \mathrm{m}$. 

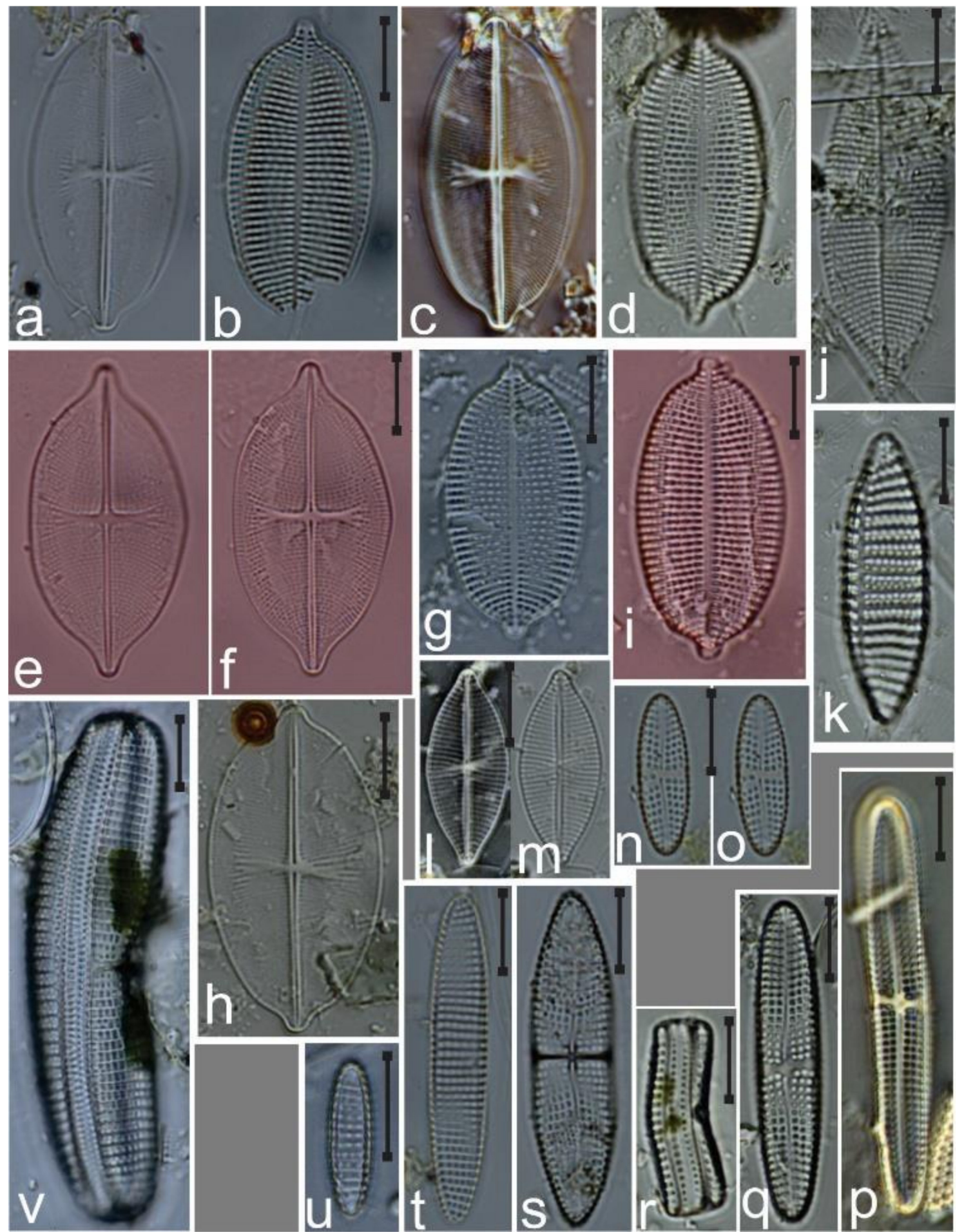

Figure 22. (a-i) Achnanthes citronella; $(\mathbf{j}, \mathbf{1}, \mathbf{m})$ A. cf. fimbriata; $(\mathbf{k}, \mathbf{v})$ A. subconstricta; $(\mathbf{n}, \mathbf{0}, \mathbf{u})$ A. parvula; $(\mathbf{p}, \mathbf{q})$ A. groenlandica var. phinneyi; $(\mathbf{r}, \mathbf{t})$ A. pseudogroenlandica; $(\mathbf{s})$ A. yaquinensis. Scale bar $=10 \mu \mathrm{m}$. 

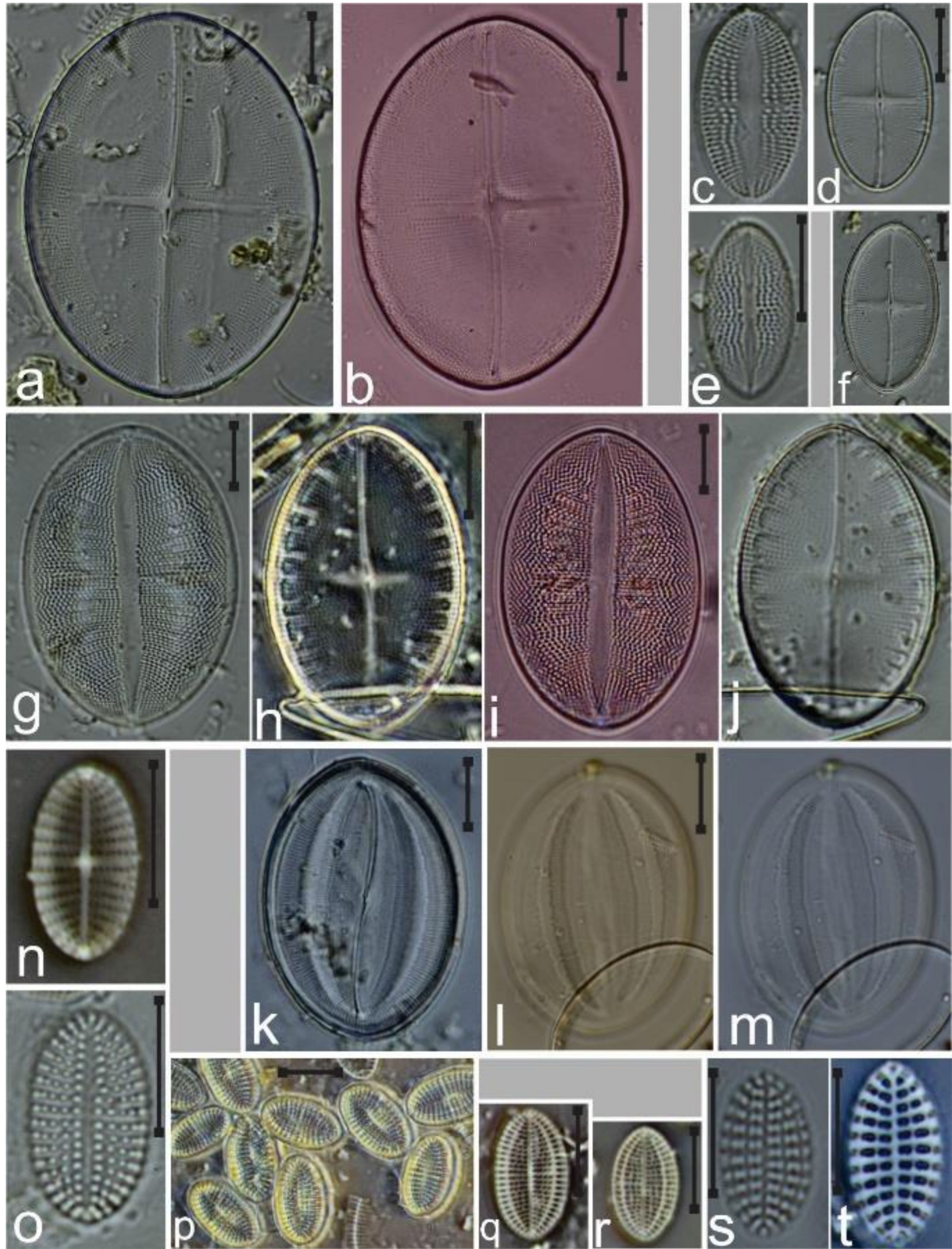

Figure 23. (a-j) Cocconeis dirupta raphe and sternum valves at different focal planes and showing range in size; $(\mathbf{k}-\mathbf{m})$ C. heteroidea; $(\mathbf{n}-\mathbf{p})$ C. scutellum var. parva, images $\mathbf{n}$ and $\mathbf{o}$, raphe and sternum valves, respectively; (q,r) C. californica; $(\mathbf{s}, \mathbf{t})$ Amphicocconeis disculoides. Images $\mathbf{q}-\mathbf{t}$ sternum valves. Scale bar $=10 \mu \mathrm{m}$. 


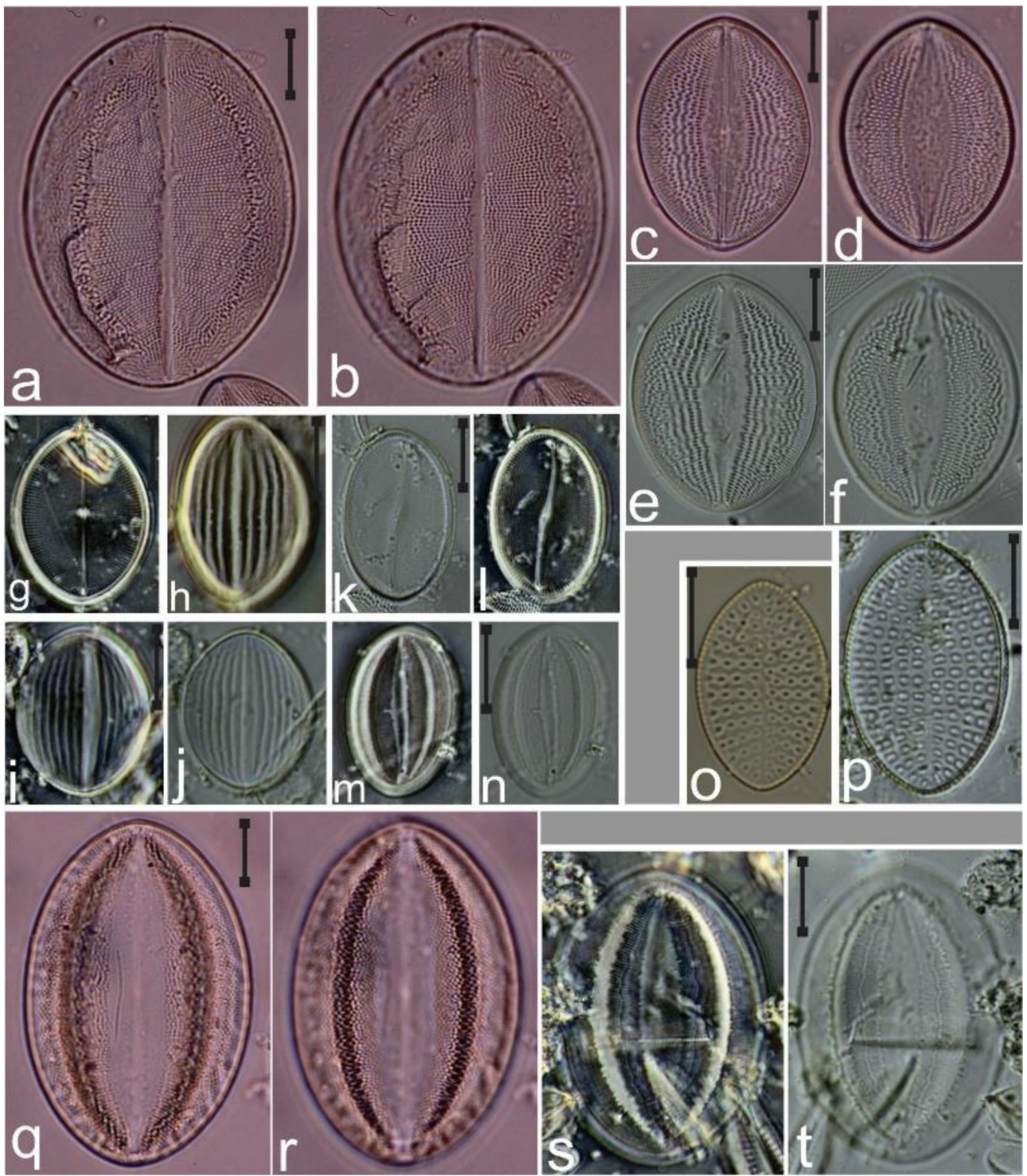

Figure 24. (a,b) Cocconeis lineata; (c-f) C. contermina raphe valves at different focal planes; $(\mathbf{g}-\mathbf{j})$ C. convexa, image $\mathbf{g}$ raphe valve, images $\mathbf{h}-\mathbf{j}$ sternum valves; $(\mathbf{k}-\mathbf{n})$ C. krammeri, images $\mathrm{k}$ and $\mathbf{l}$ raphe, images $\mathrm{m}$ and sternum valves; $(\mathbf{o}, \mathbf{p})$ C. guttata; $(\mathbf{q}-\mathbf{t})$ C. pseudomarginata. Scale bar $=10 \mu \mathrm{m}$. 


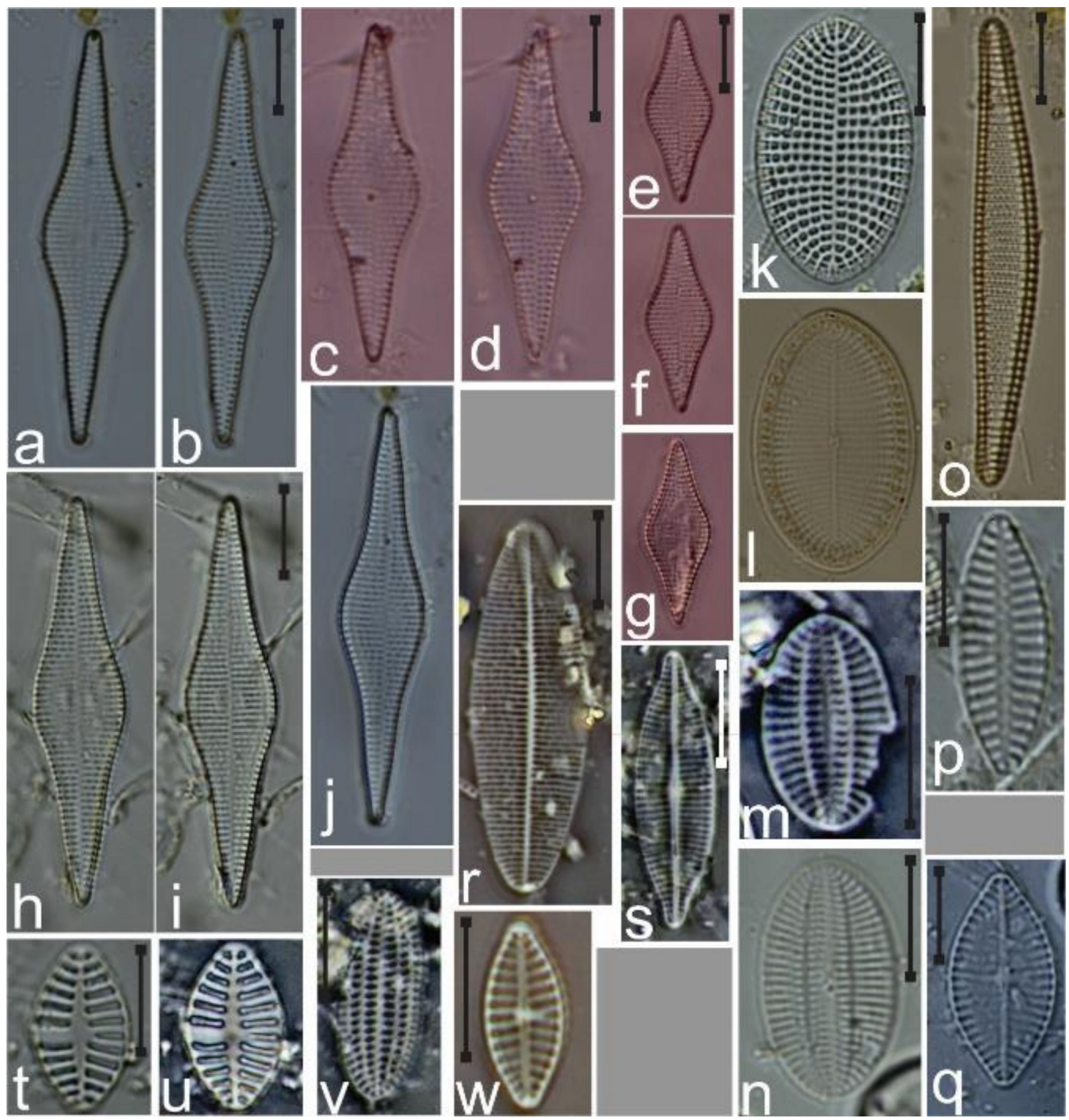

Figure 25. (a-j) Glyphodesmis rhombica valves at different focal planes and showing range in size; (k,l) Cocconeis scutellum sternum and raphe valves, respectively; $(\mathbf{m}, \mathbf{n})$ C. peltoides; (o) Fragilariopsis doliolus; (p) Planothidium lilljeborgei sternum valve; $(\mathbf{q}, \mathbf{t}, \mathbf{u})$ P. delicatulum raphe and sternum valves; (r) P. polare sternum valve; (s) P. campechianum raphe valve; (v) Amphicocconeis discrepans sternum valve; (w) Planothidium hauckianum raphe valve. Scale bar $=10 \mu \mathrm{m}$. 


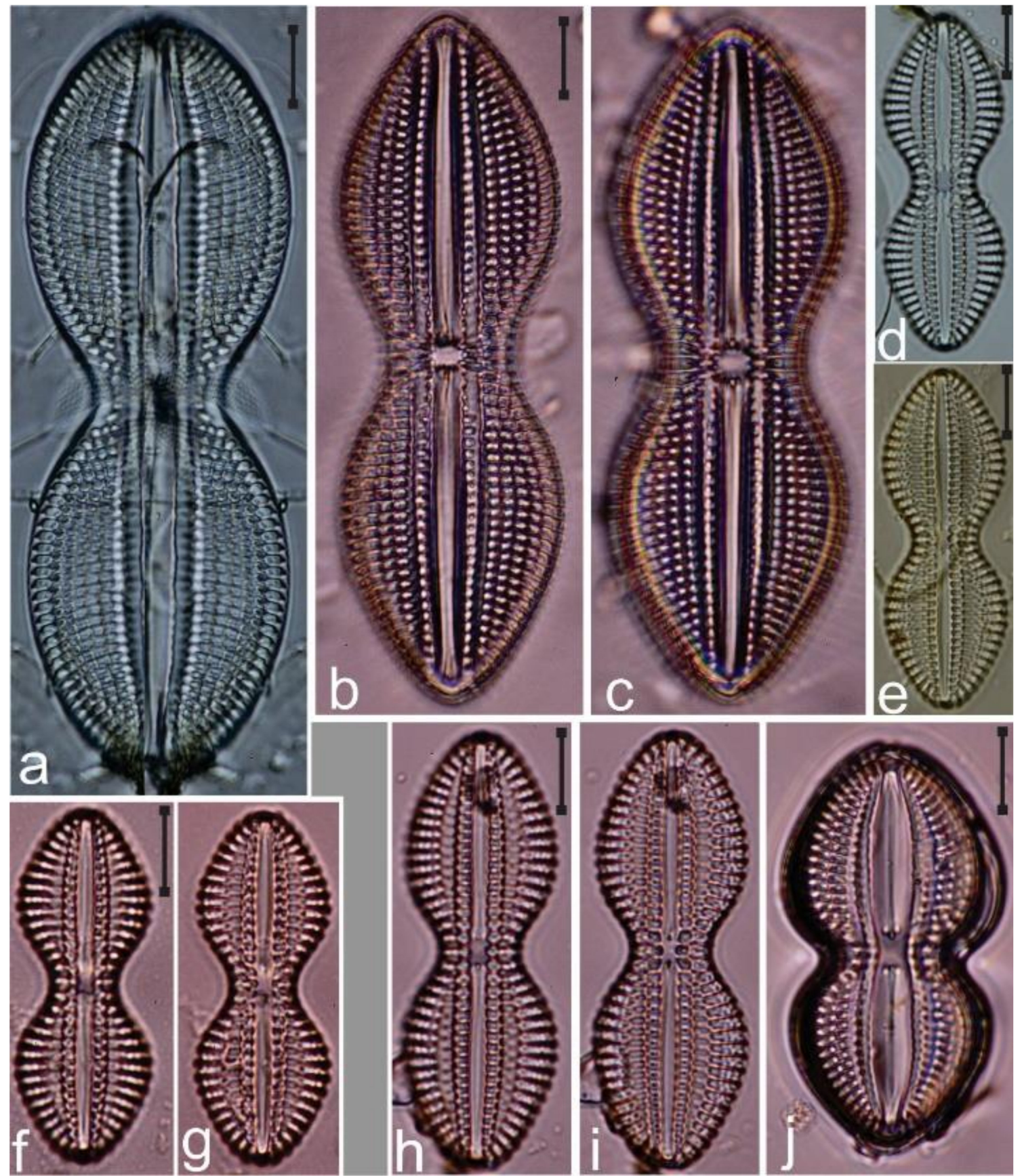

Figure 26. (a) Diploneis bombus; $(\mathbf{b}, \mathbf{c})$ D. chersonensis; $(\mathbf{d}-\mathbf{i})$ D. crabro valves at different focal planes and showing range in size; $(\mathbf{j})$ D. gruendleri. Scale bar $=10 \mu \mathrm{m}$. 


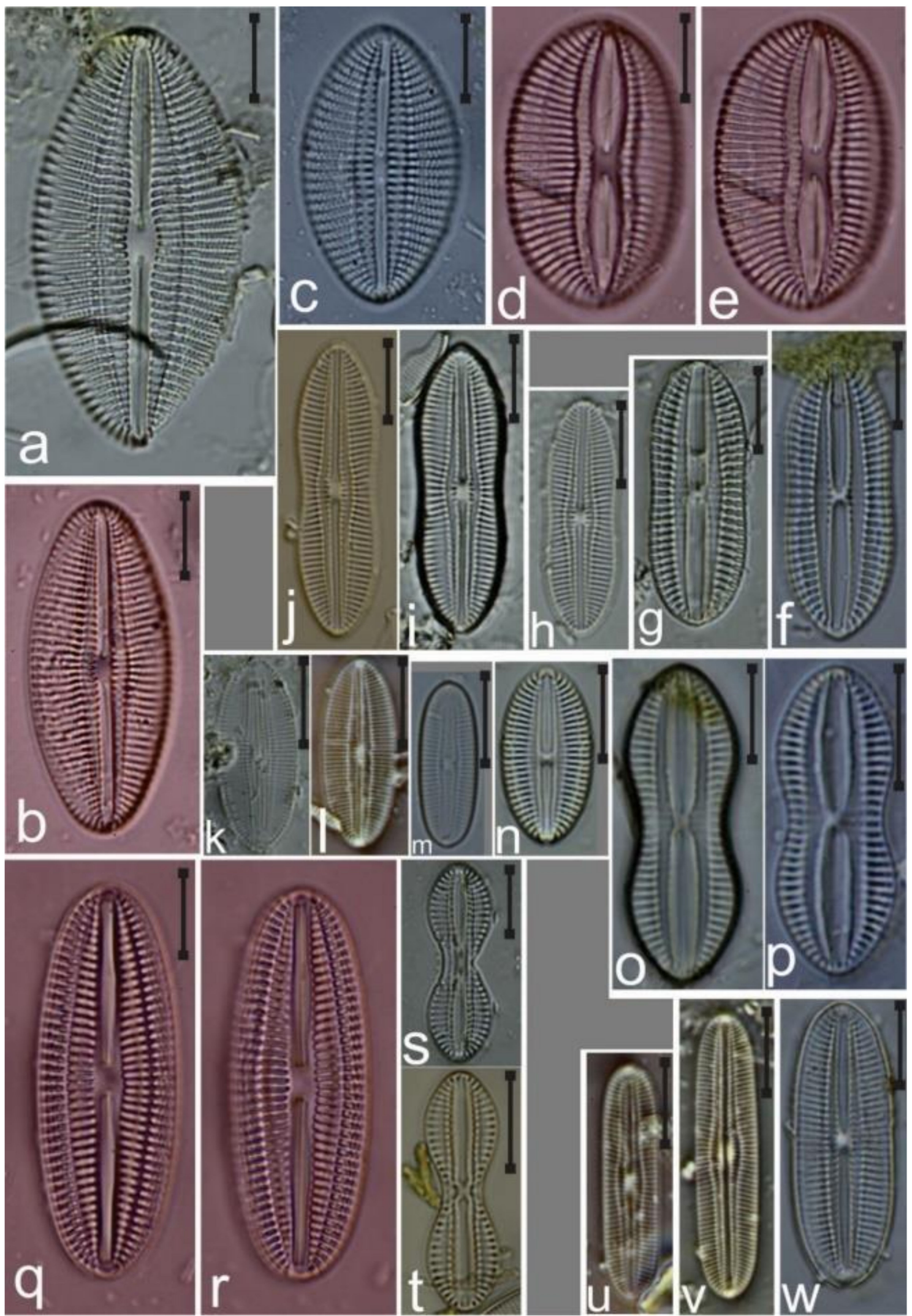

Figure 27. (a-c) Diploneis smithii; $(\mathbf{d}, \mathbf{e})$ D. suborbicularis; $(\mathbf{f}-\mathbf{j})$ D. vacillans var. renitens; $(\mathbf{k}, \mathbf{l})$ D. littoralis var. clathrata; $(\mathbf{m}, \mathbf{u}, \mathbf{v})$ D. vacillans var. vacillans; $(\mathbf{n})$ D. papula; $(\mathbf{o}, \mathbf{p})$ D. incurvata; $(\mathbf{q}, \mathbf{r})$ D. nitescens; (s) D. novaeseelandiae; (t) D. suspecta; $(\mathbf{w})$ D. littoralis. Scale bar $=10 \mu \mathrm{m}$. 


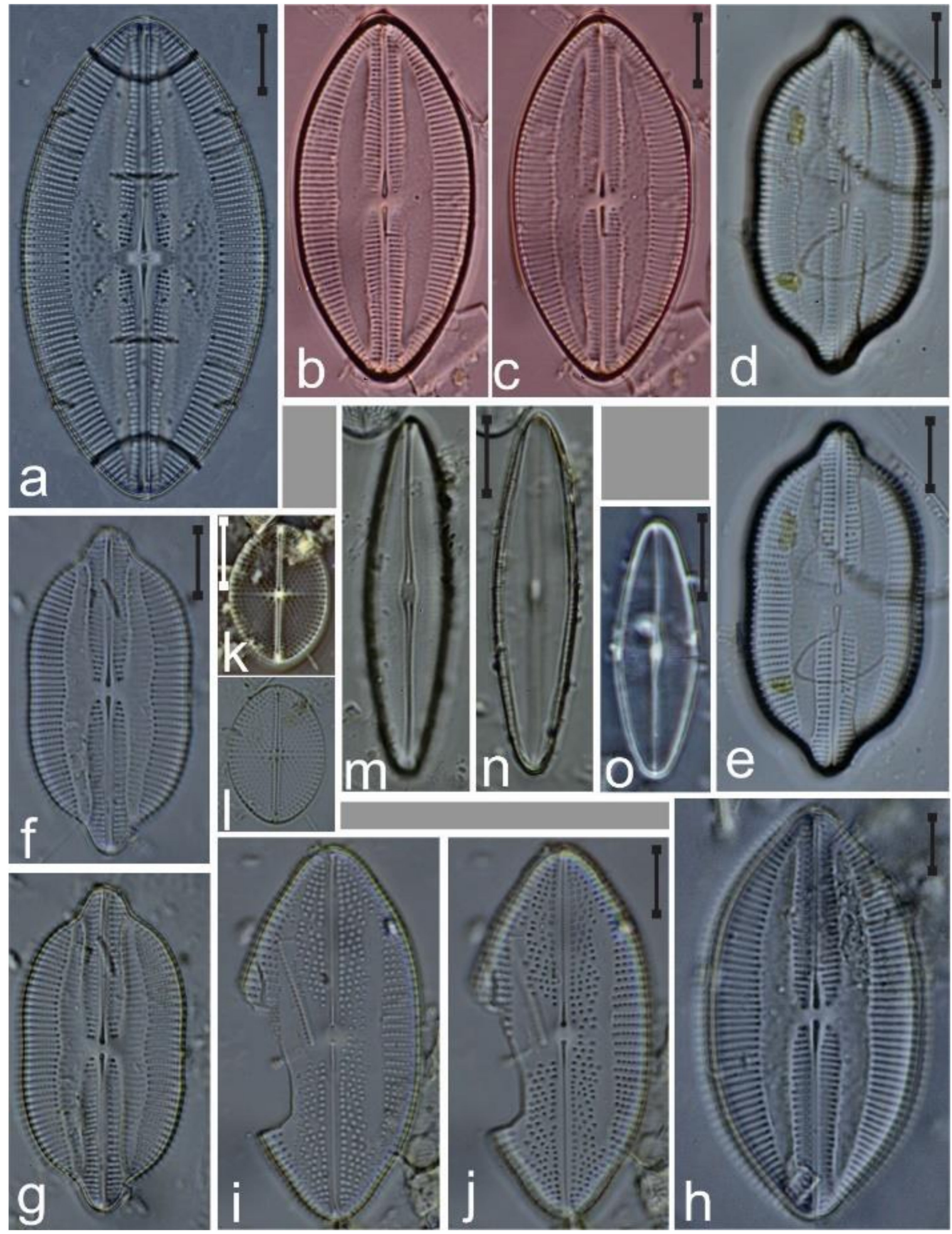

Figure 28. (a-c) Lyrella hennedyi; $(\mathbf{d}-\mathbf{g})$ L. clavata var. caribaea; $(\mathbf{h})$ L. atlantica; $(\mathbf{i}, \mathbf{j})$ L. approximatoides; $(\mathbf{k}, \mathbf{l})$ Mastogloia binotata; $(\mathbf{m}, \mathbf{n})$ M. ciskeiensis; $(\mathbf{o})$ M. chersonensis. Scale bar $=10 \mu \mathrm{m}$. 


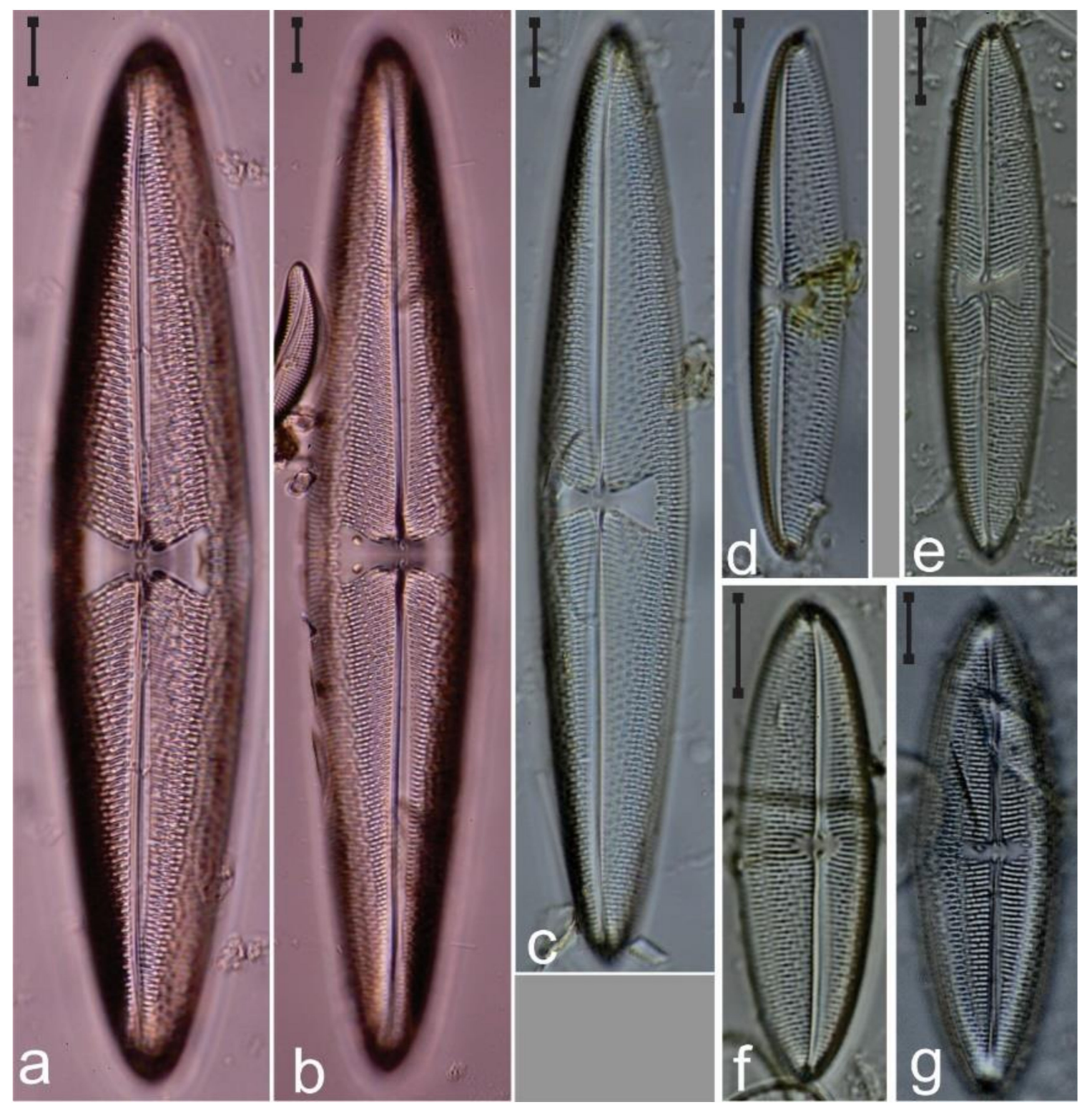

Figure 29. (a-e) Trachyneis aspera valves at different focal planes and showing range in size; (f,g) T. velata. Scale bar $=10 \mu \mathrm{m}$. 

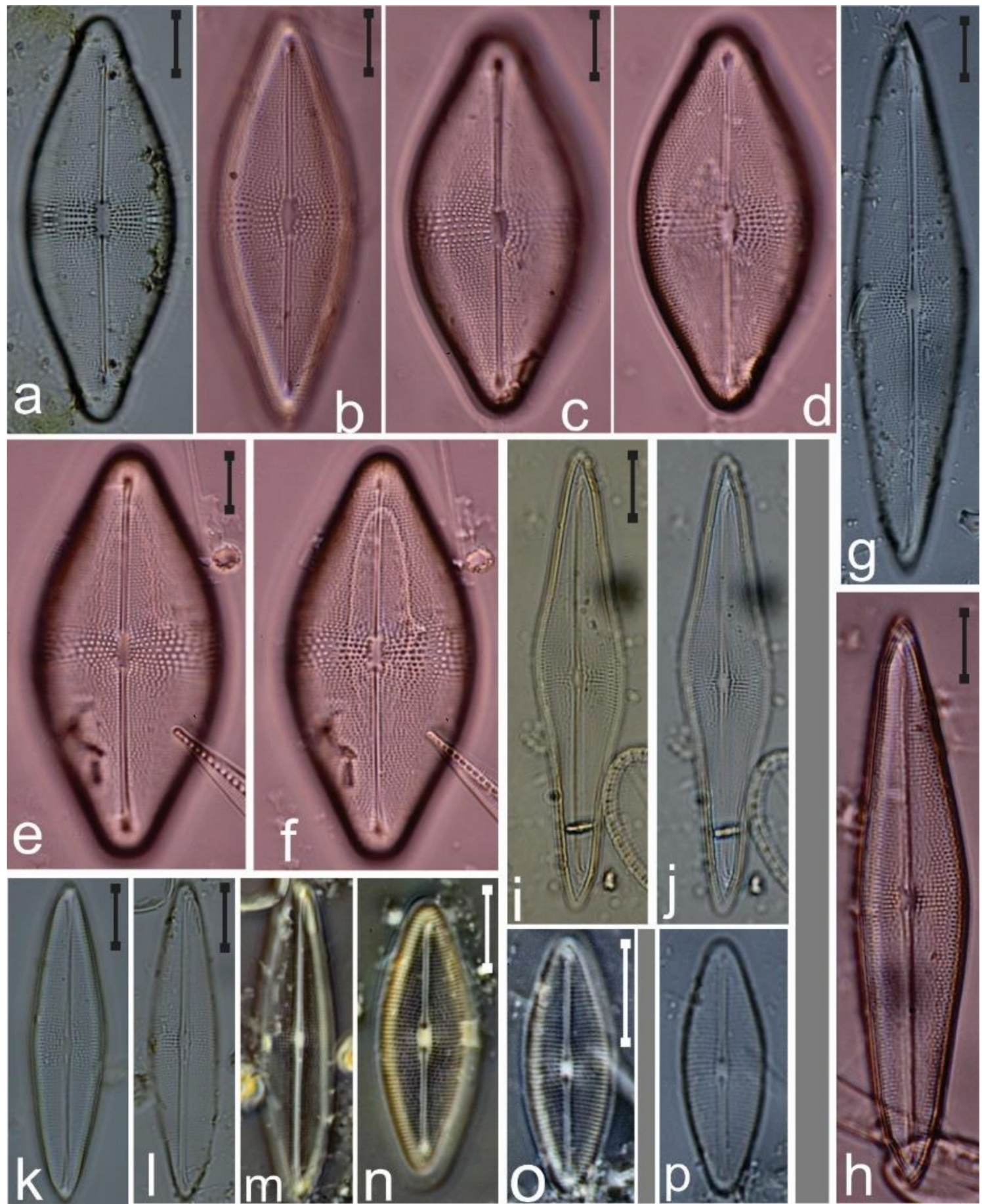

Figure 30. (a-f) Parlibellus weissflogii; $(\mathbf{g}, \mathbf{h}, \mathbf{k}-\mathbf{m})$ P. rhombicula; $(\mathbf{i}, \mathbf{j})$ P. rhombicus; $(\mathbf{n}-\mathbf{p})$ P. delognei. Scale bar $=10 \mu \mathrm{m}$. 


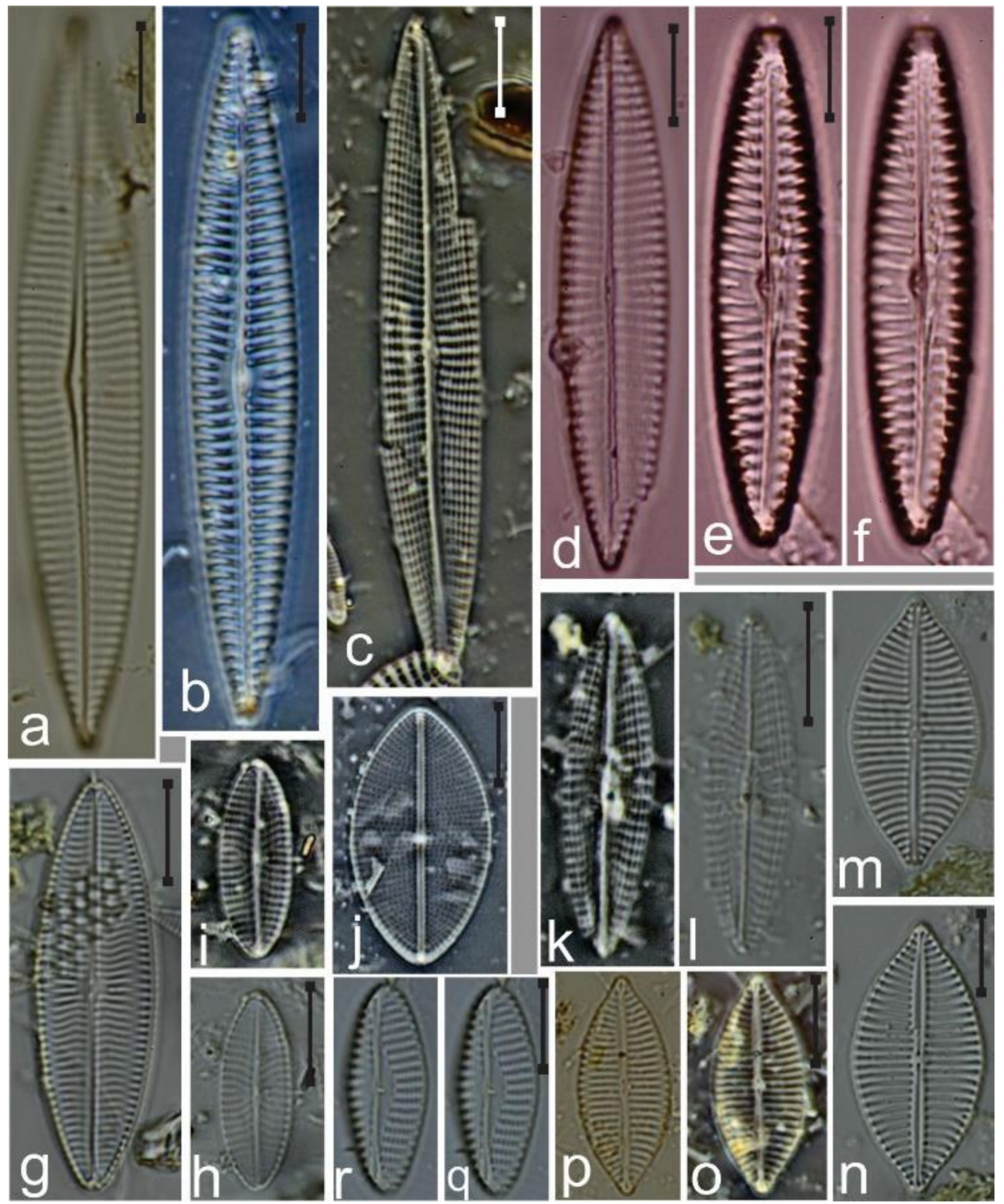

Figure 31. (a) Navicula transitans; (b) N. pennata; (c,k,l) N. pavillardii; (d) N. arenaria var. rostellata; $(\mathbf{e}, \mathbf{f})$ N. cancellata; $(\mathbf{g})$ N. johannrossii; $(\mathbf{h}, \mathbf{i})$ N. lusoria; $(\mathbf{j})$ N. cluthensis; $(\mathbf{m}-\mathbf{p}) N$. diversistriata; $(\mathbf{q}, \mathbf{r}) N$. valida var. minuta. Scale bar $=10 \mu \mathrm{m}$. 

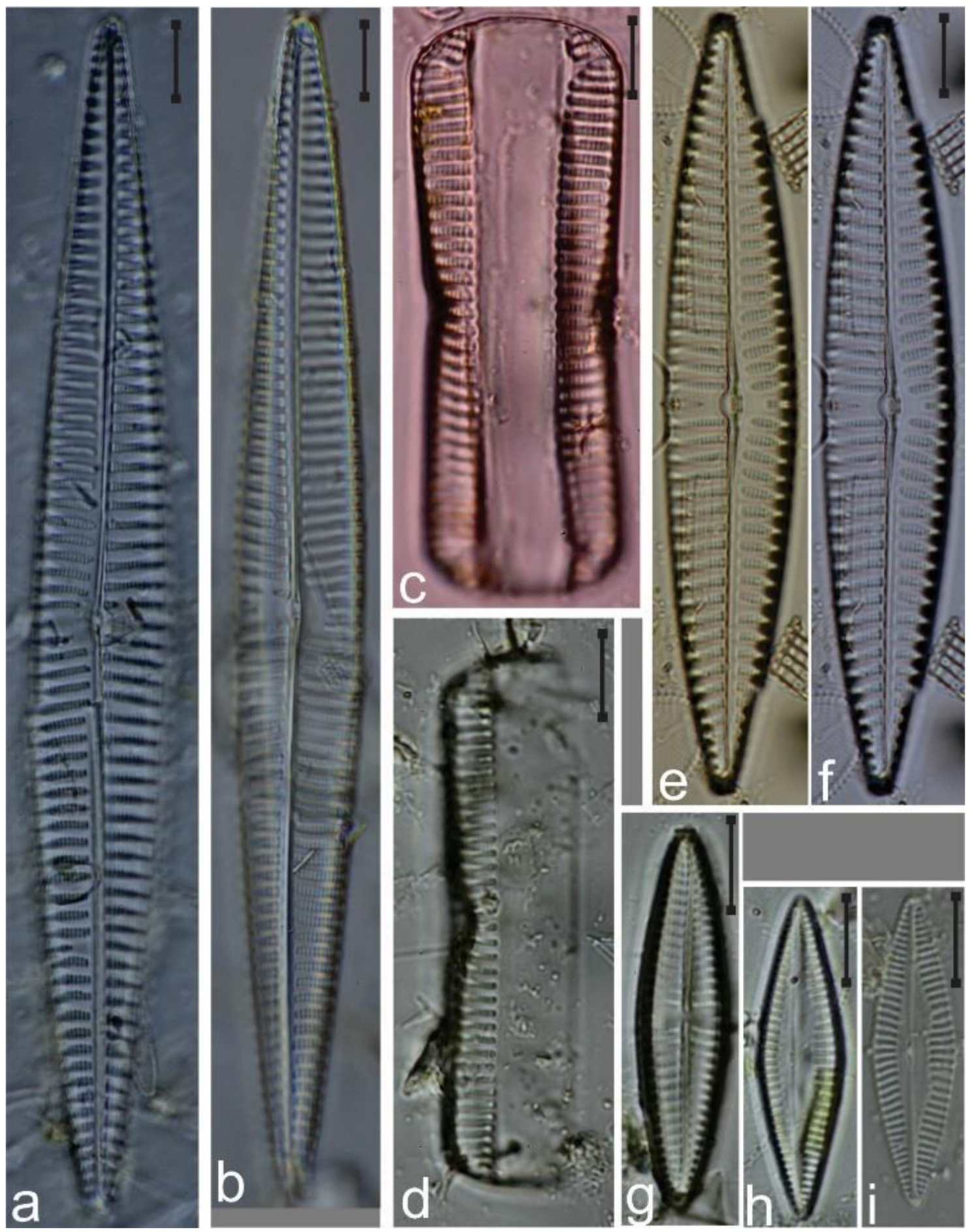

Figure 32. (a,b) Navicula longa var. irregularis; $(\mathbf{c}, \mathbf{g})$ N. cancellata, images $\mathrm{c}$ and $\mathrm{g}$ whole frustule and valve view, respectively; (d) N. pennata valve in girdle view; $(\mathbf{e}, \mathbf{f}) \mathrm{N}$. longa var. longa; $(\mathbf{h}, \mathbf{i}) \mathrm{N}$. palpebralis var. angulosa. Scale bar $=10 \mu \mathrm{m}$. 

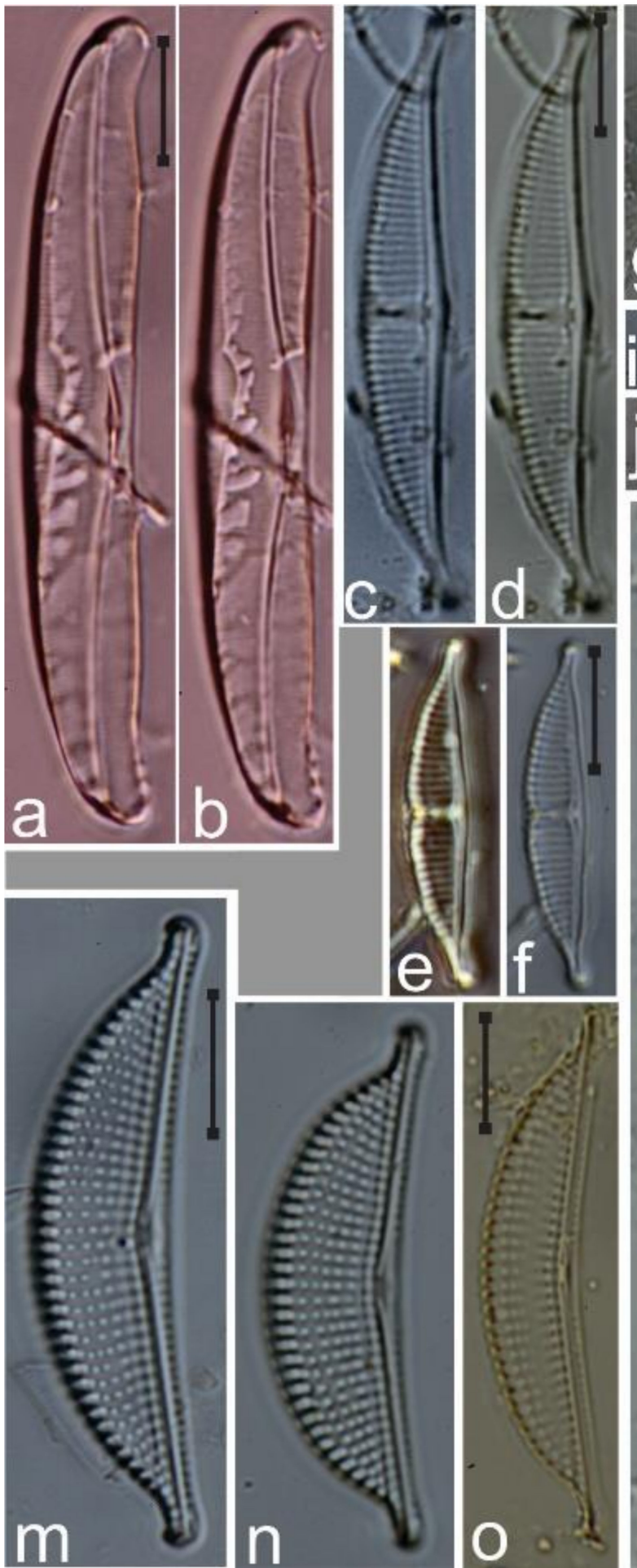

Figure 33. (a,b) Amphora studeri; (c-f) Halamphora cuneata; (g-1) Amphora angustissima; $(\mathbf{m}-\mathbf{q})$ Halamphora costata. Scale bar $=10 \mu \mathrm{m}$.
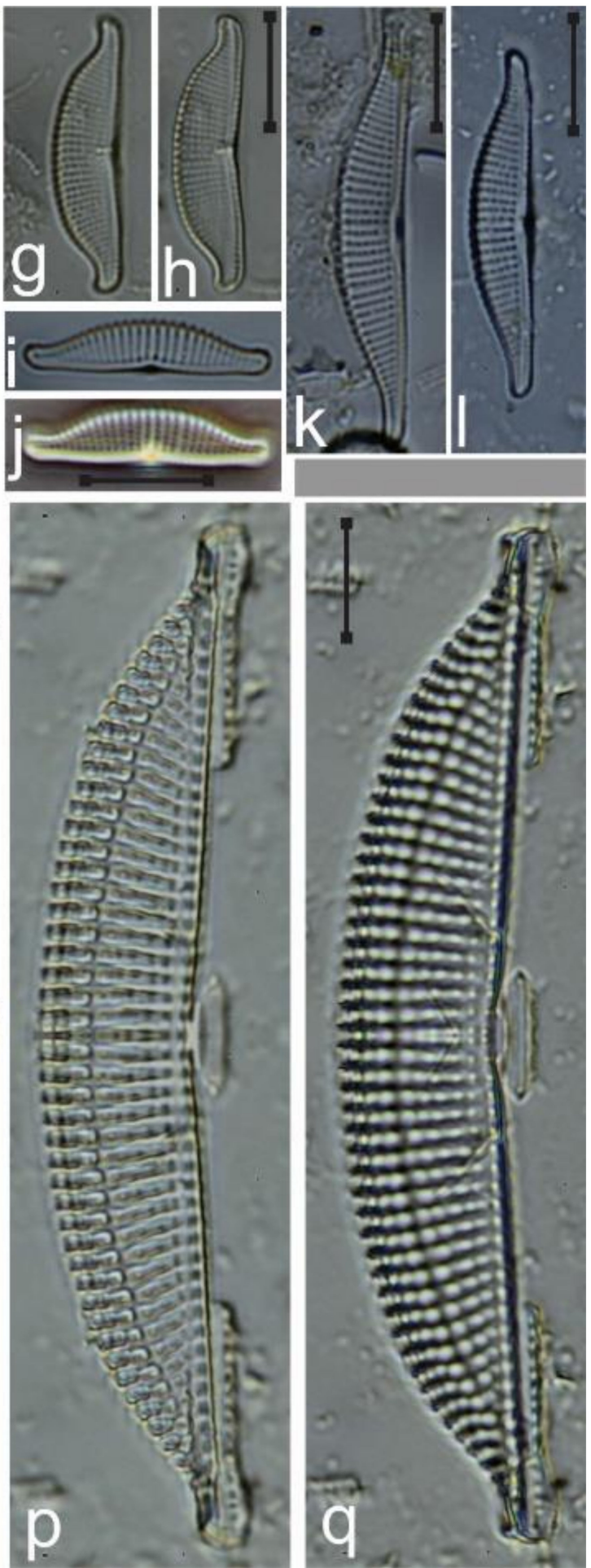

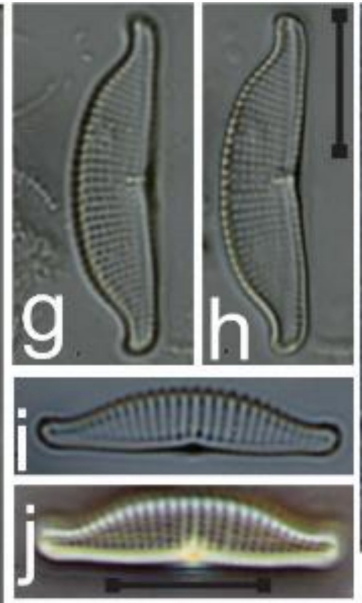




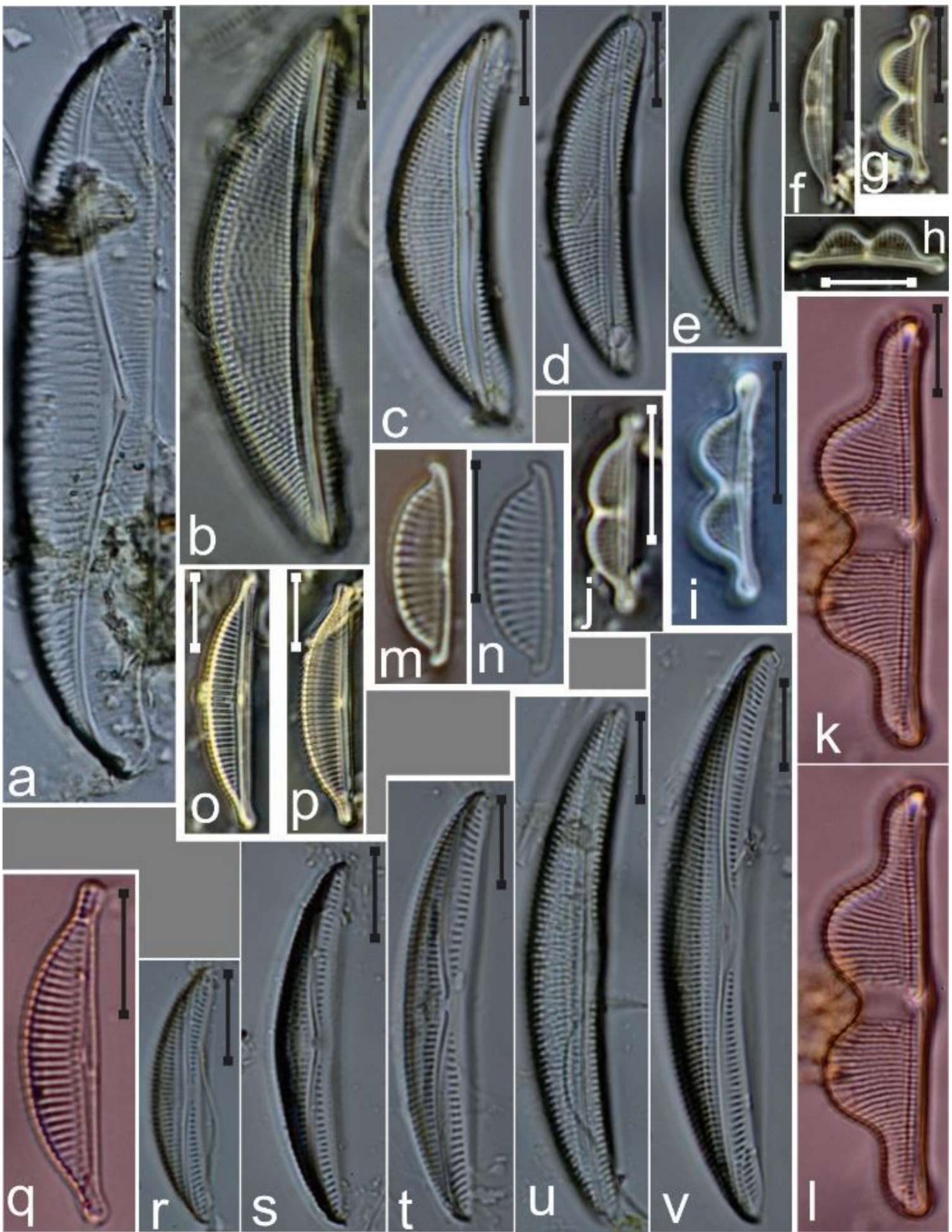

Figure 34. (a) Amphora spectabilis; (b-e) A. immarginata; (f-h, $\mathbf{j})$ A. bigibba; (i) Halamphora capitata; $(\mathbf{k}, \mathbf{l})$ Amphora bigibba var. interrupta; $(\mathbf{m}, \mathbf{n})$ Halamphora wisei; $(\mathbf{o}, \mathbf{p})$ H. coffeiformis; (q) H. acutiuscula; (r) Amphora proteus var. contigua; $(\mathbf{s , t})$ A. marina; $(\mathbf{u}, \mathbf{v})$ A. proteus. Scale bar $=10 \mu \mathrm{m}$. 


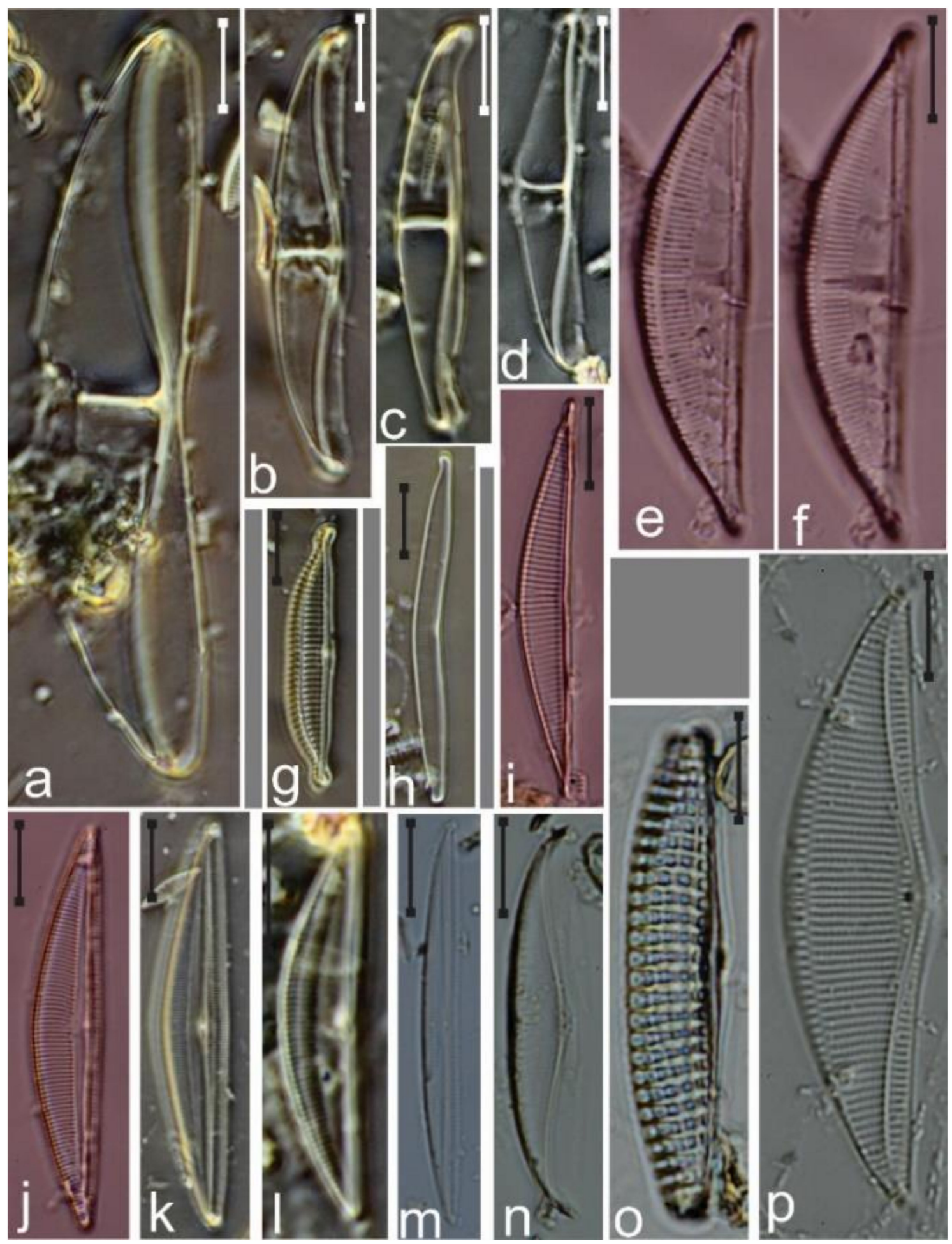

Figure 35. (a,b) Amphora cingulata; (c,d) A. laevissima; (e,f) A. graeffeana; (g) Halamphora exigua; (h) Amphora americana; (i) A. cf. cymbamphora; (j) Seminavis robusta; (k) S. basilica; (1) Amphora beaufortiana; (m) Seminavis barbarae; (n) Amphora obtusa; (o) A. crassa; (p) Tetramphora securicula. Scale bar $=10 \mu \mathrm{m}$. 

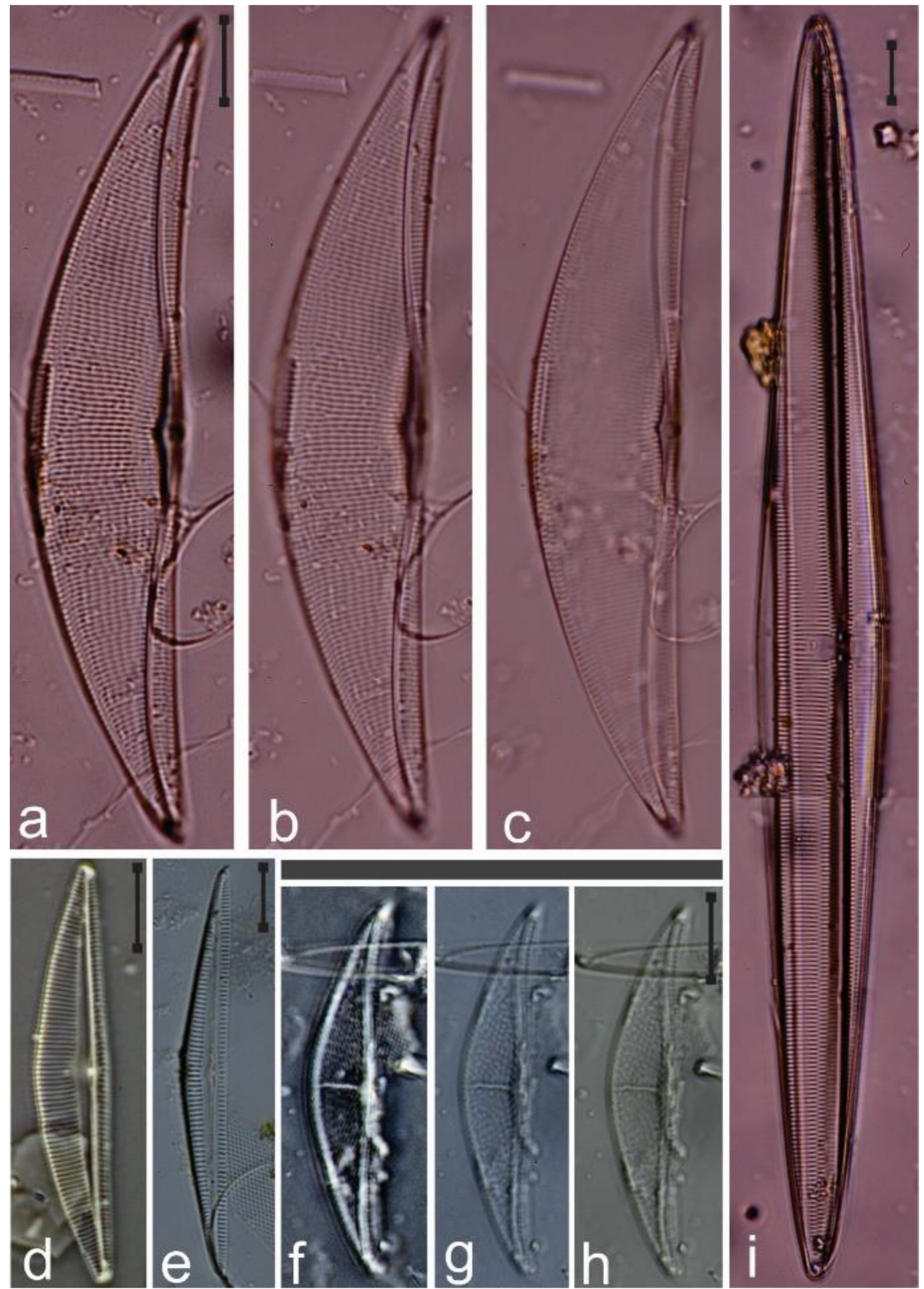

Figure 36. (a-c) Tetramphora intermedia in different focal planes; (d) Seminavis robusta; (e) S. macilenta; (f-h) Tetramphora decusata; (i) Plagiotropis australis. Scale bar $=10 \mu \mathrm{m}$. 

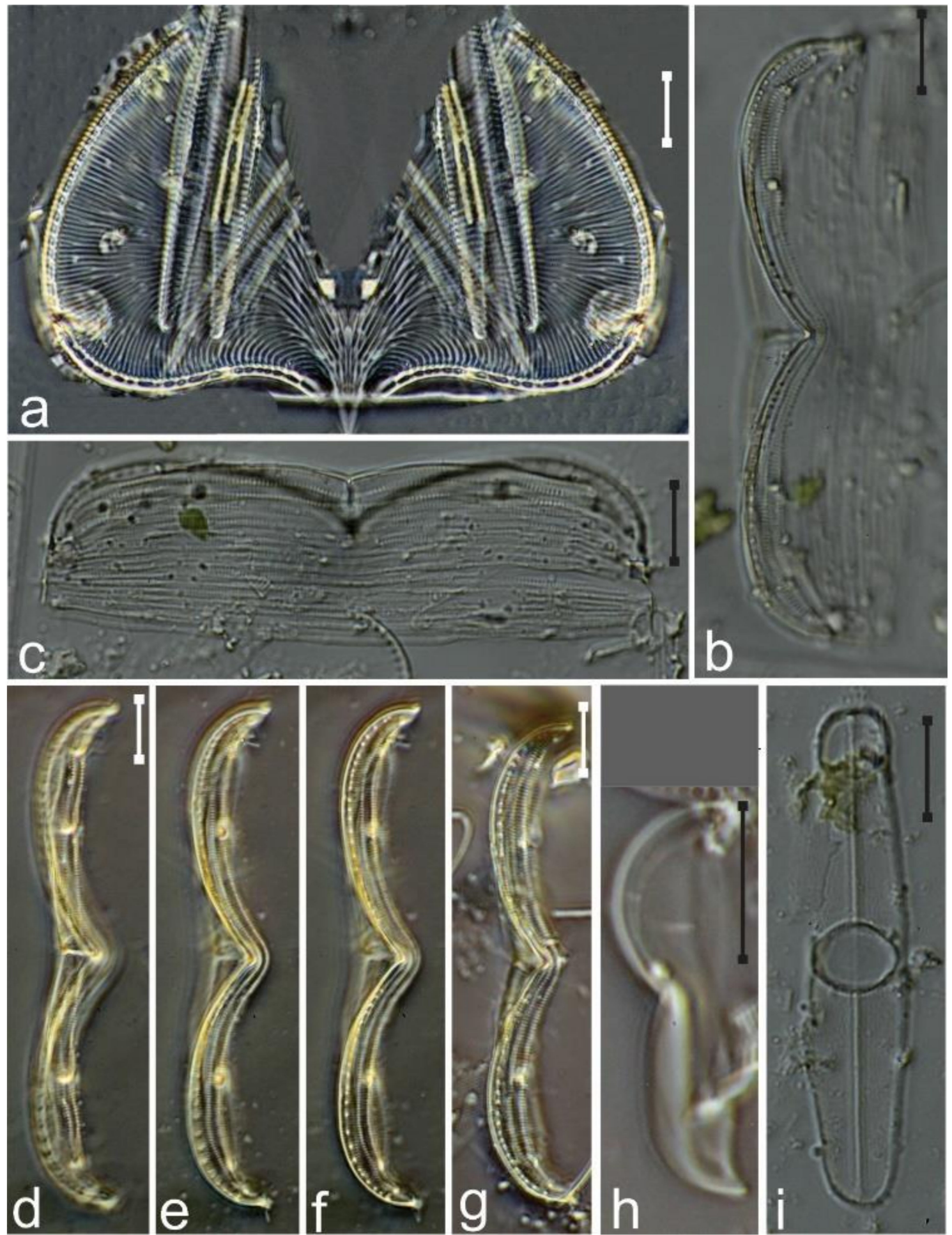

Figure 37. (a) Auricula flabelliformis; (b-g) A. pulchra; (h) Amphiprora pseudoduplex; (i) Cyclophora tenuis. Scale bar $=10 \mu \mathrm{m}$. 


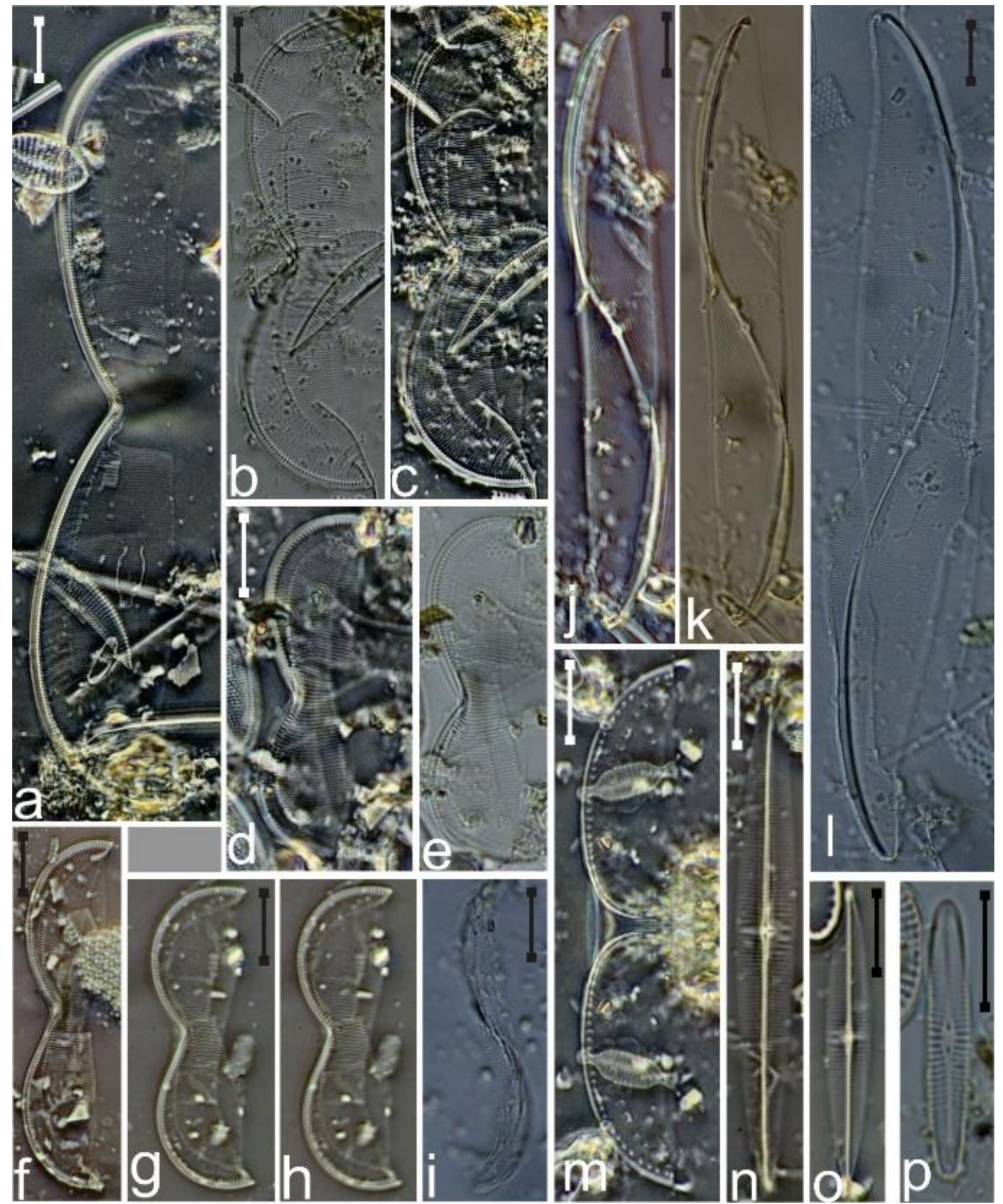

Figure 38. (a-i) Entomoneis paludosa; (j-1) Donkinia carinata; (m) Thalassiophysa hyalina; $(\mathbf{n}, \mathbf{o})$ Proschkinia complanata; (p) Rhoicosphenia abbreviata. Scale bar $=10 \mu \mathrm{m}$. 

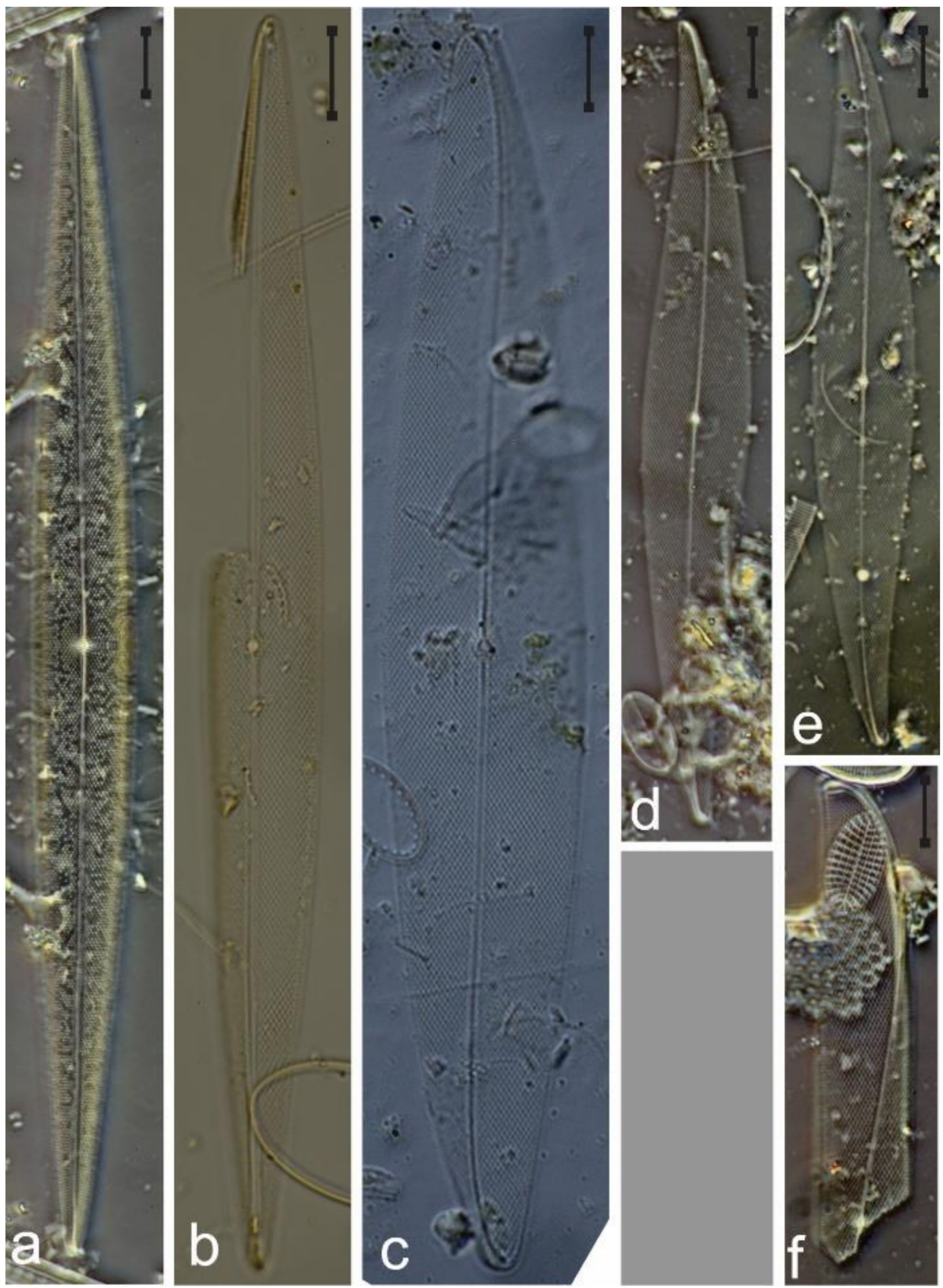

Figure 39. (a,b) Pleurosigma patagonicum var. paucistriatum; (c) P. rigidum; (d,e) P. cf. gracile; (f) P. subsalinum. Scale bar $=10 \mu \mathrm{m}$. 


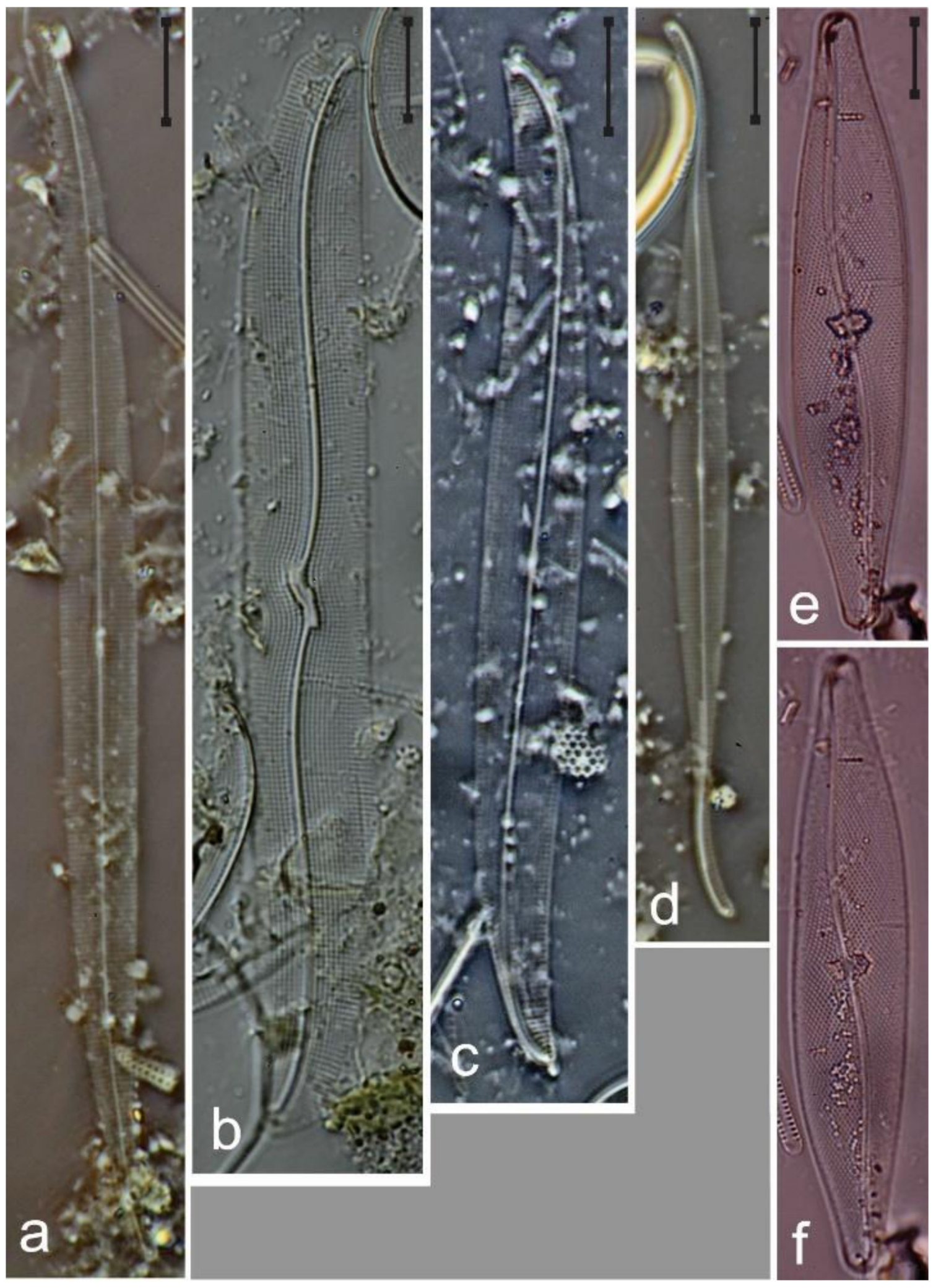

Figure 40. (a) Gyrosigma tenuissimum var. hyperboreum; (b) G. balticum; (c) G. peisonis; (d) G. reversum; (e,f) Pleurosigma naviculaceum. Scale bar $=10 \mu \mathrm{m}$. 


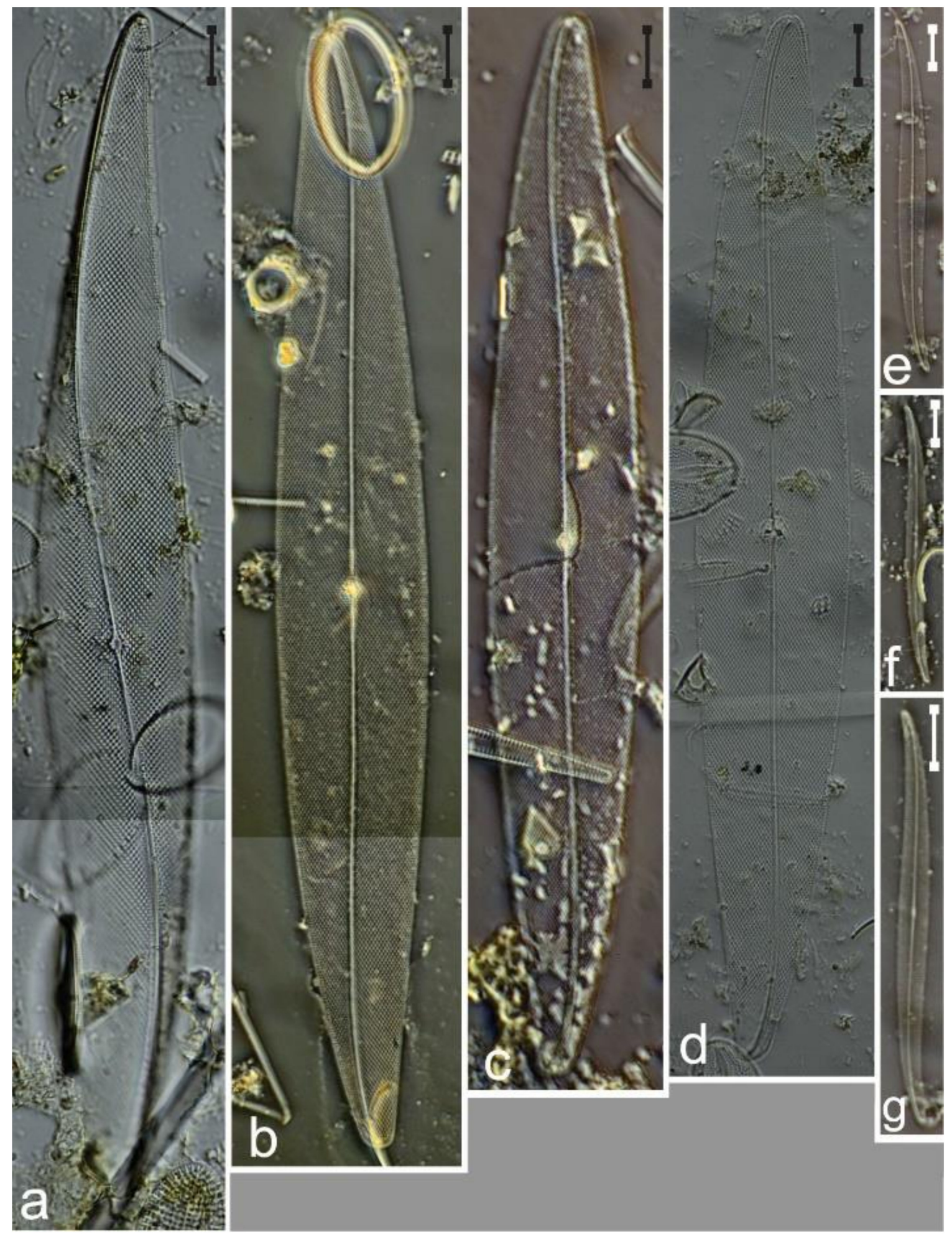

Figure 41. (a) Pleurosigma formosum; (b-d) P. rigidum; (e-g) Gyrosigma parvulum. Scale bar $=10 \mu \mathrm{m}$. 


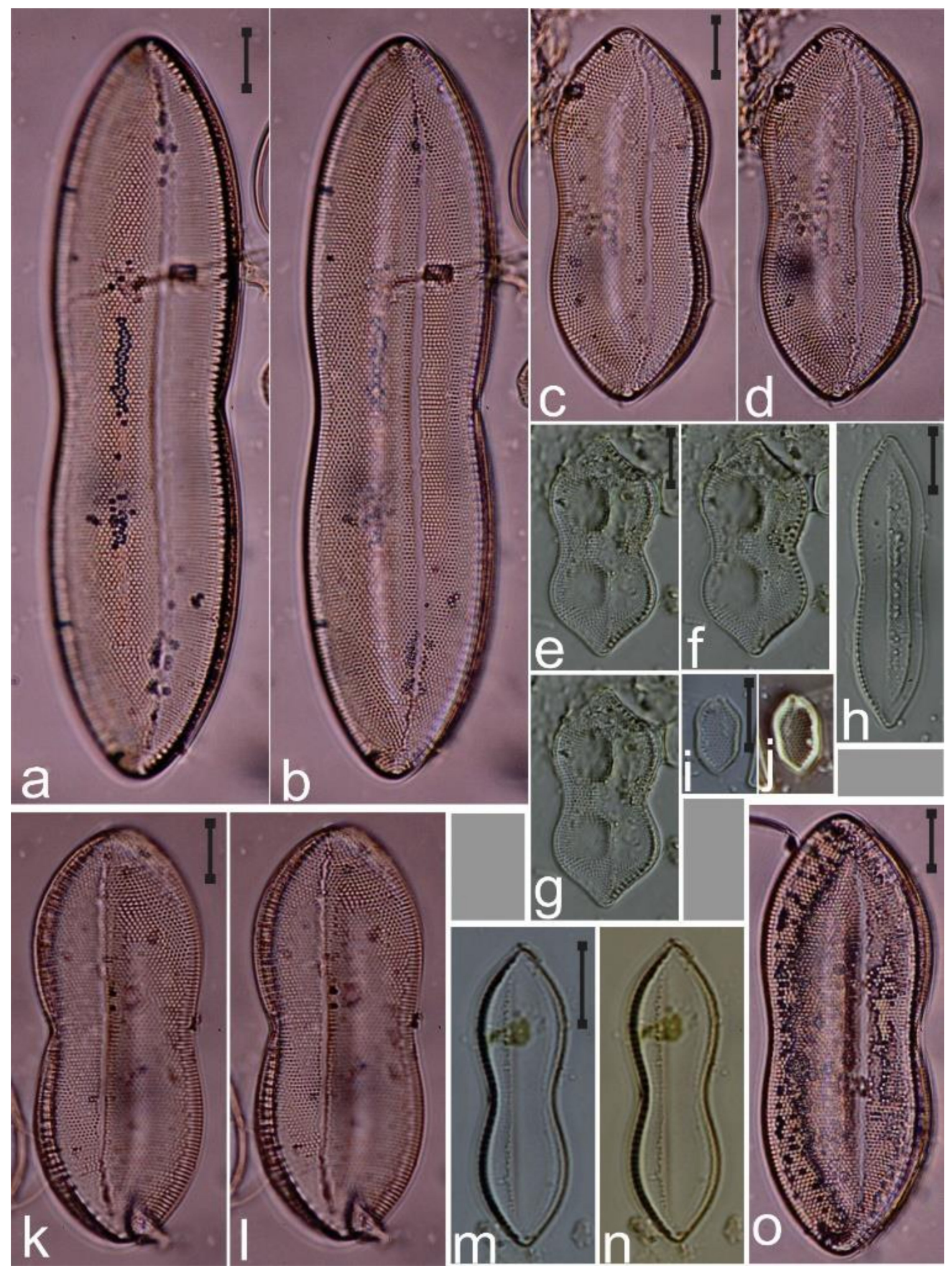

Figure 42. (a-d,k,1,o) Psammodictyon panduriforme; (e-g) P. pustulatum; (h) P. roridum; $(\mathbf{i}, \mathbf{j})$ P. panduriforme var. continuum; $(\mathbf{m}, \mathbf{n})$ Nitzschia carnicobarica. Scale bar $=10 \mu \mathrm{m}$. 

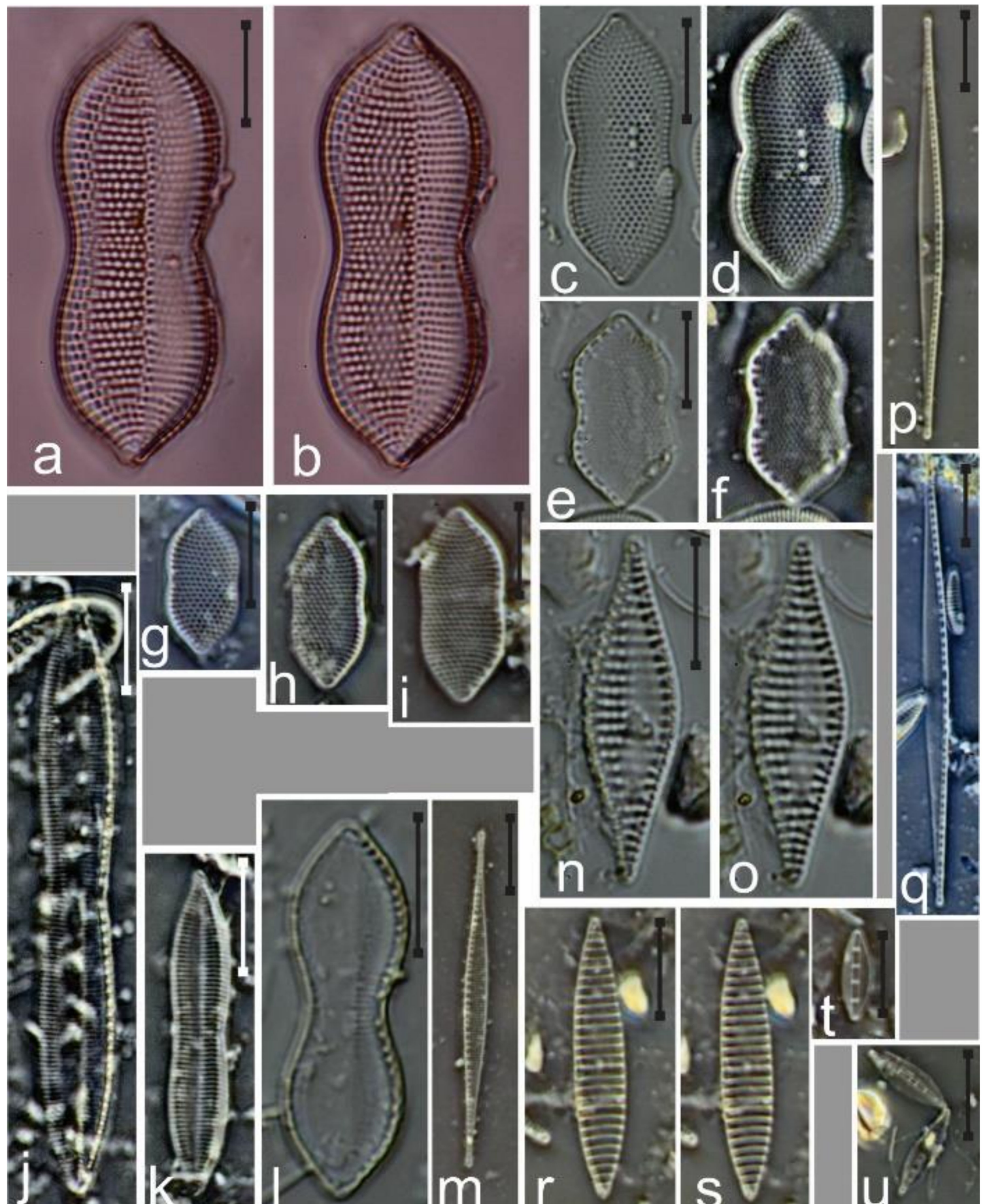

e
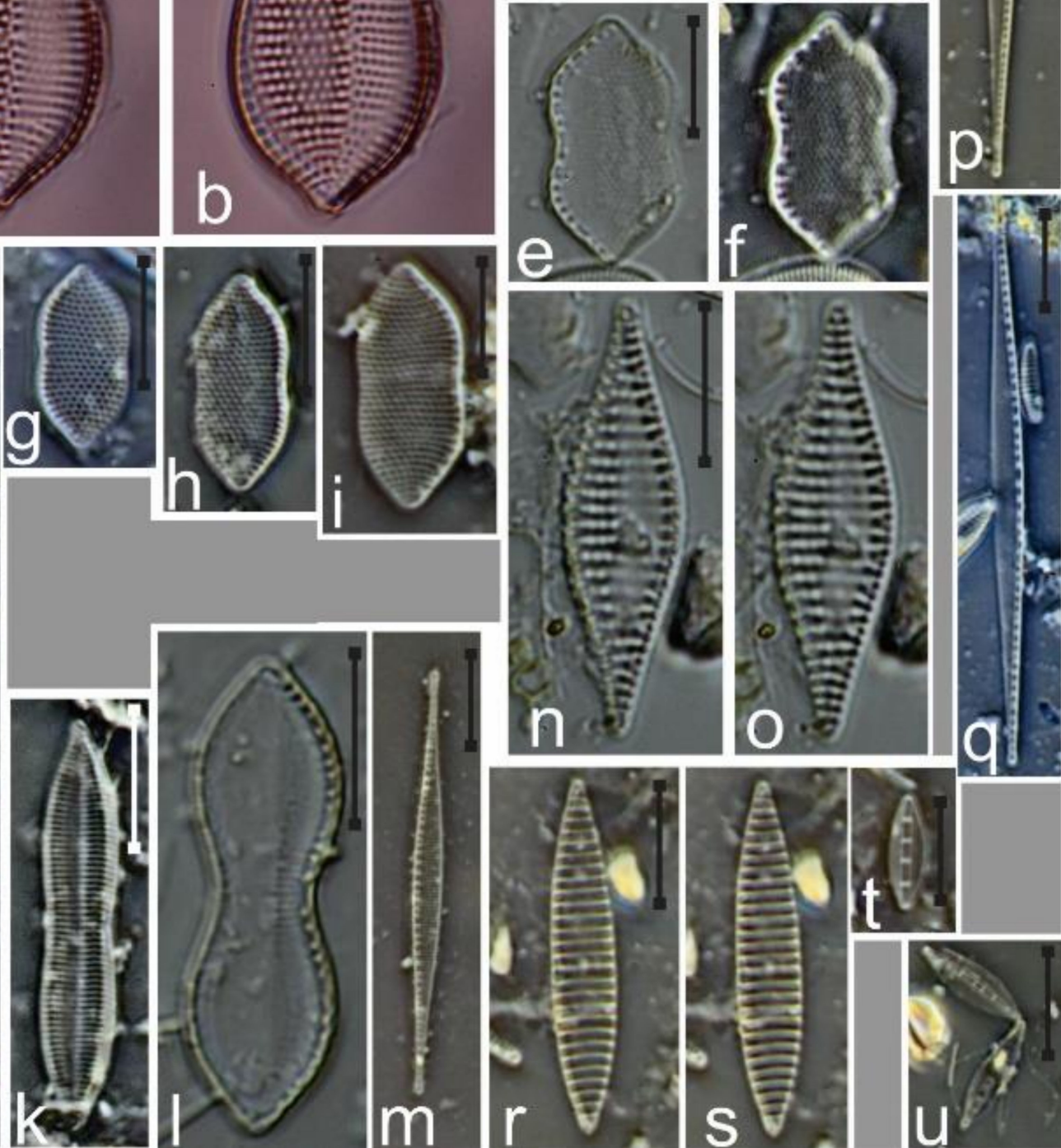

Figure 43. (a-d,g,h) Tryblionella coarctata; (e,f,i) T. bathurstensis; (j,k) T. hungarica; (1) Nitzschia carnicobarica; $(\mathbf{m}, \mathbf{p}, \mathbf{q})$ N. gracilis; $(\mathbf{n}, \mathbf{o})$ Tryblionella lanceola; $(\mathbf{r}, \mathbf{s})$ Nitzschia sicula; $(\mathbf{t}, \mathbf{u})$ N. incrustans. Scale bar $=10 \mu \mathrm{m}$. 


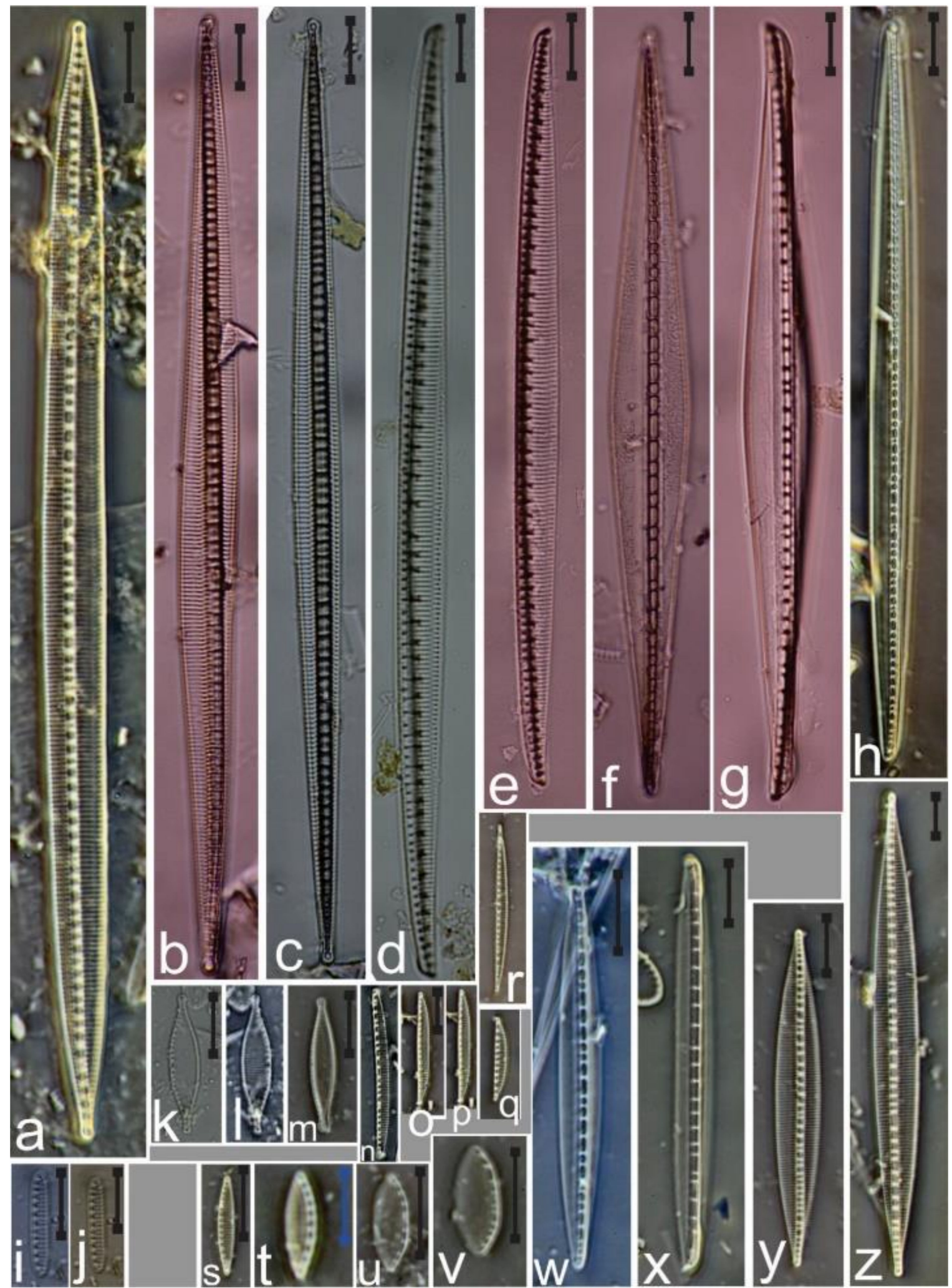

Figure 44. (a,y,z) Bacillaria socialis; (b,c) Nitzschia longa; (d,e) N. insignis; (f,g) N. angularis; $(\mathbf{h}, \mathbf{w})$ N. spathulata; $(\mathbf{i}, \mathbf{j})$ N. valdestriata; $(\mathbf{k}, \mathbf{l})$ N. bicapitata; $(\mathbf{m})$ N. agnita; $(\mathbf{n}-\mathbf{r})$ Cymbellonitzschia banzuensis; $(\mathbf{s}, \mathbf{t})$ Nitzschia frustulum; $(\mathbf{u}, \mathbf{v})$ N. amabilis; $(\mathbf{x})$ N. distans. Scale bar $=10 \mu \mathrm{m}$. 


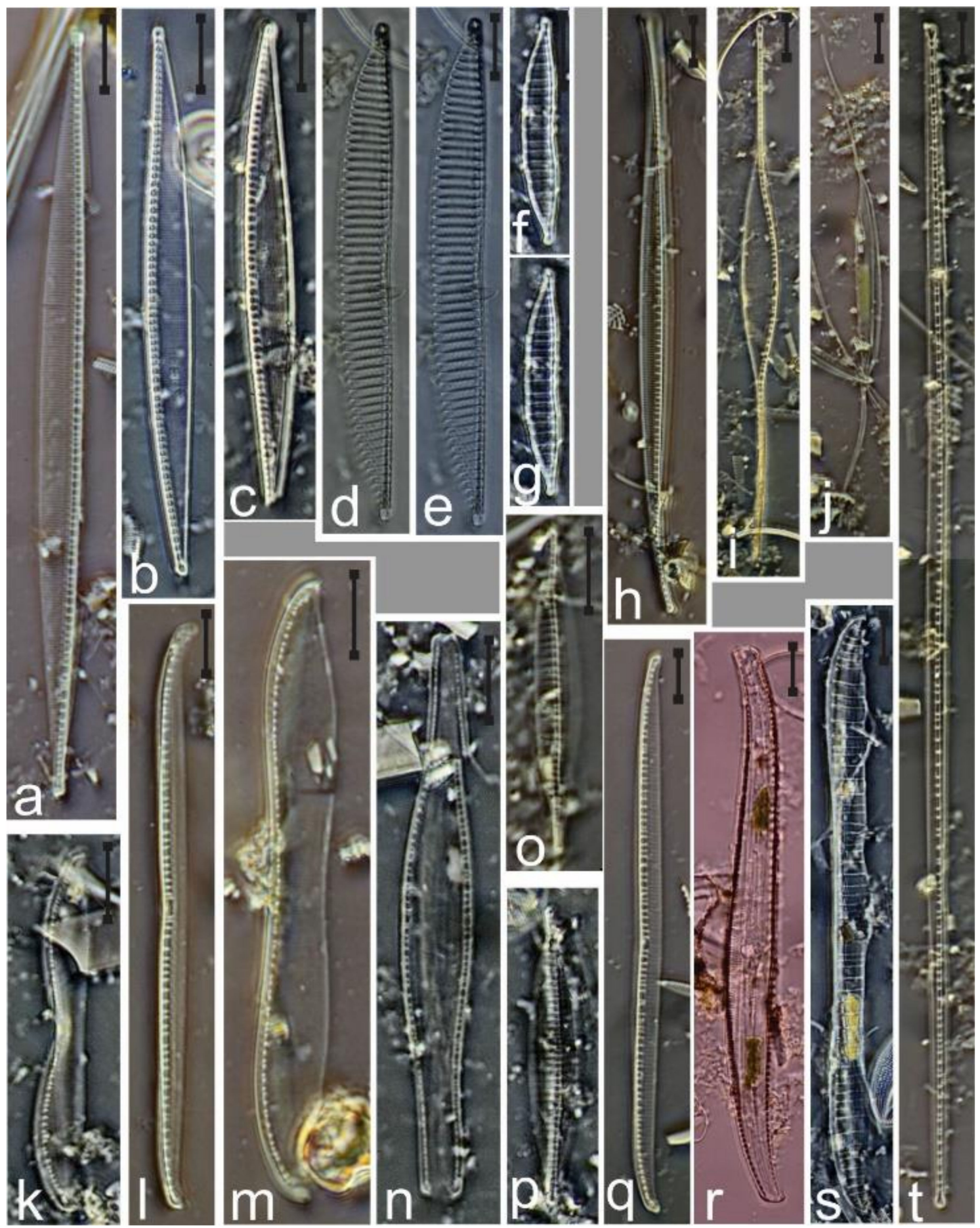

Figure 45. (a) Nitzschia fusiformis; (b,c) N. sigma; (d-g) Hantzschia marina; (h) Nitzschia incurva var. lorenziana; (i) N. longissima; (j) Cylindrotheca closterium; (k,m) Nitzschia hybrida; (1) N. linearis; (n) N. lanceolata; (o,p) N. composita; (q) N. cf. spectabilis var. americana; (r) N. insignis; (s) N. costata; (t) N. martiana. Scale bar $=10 \mu \mathrm{m}$. 

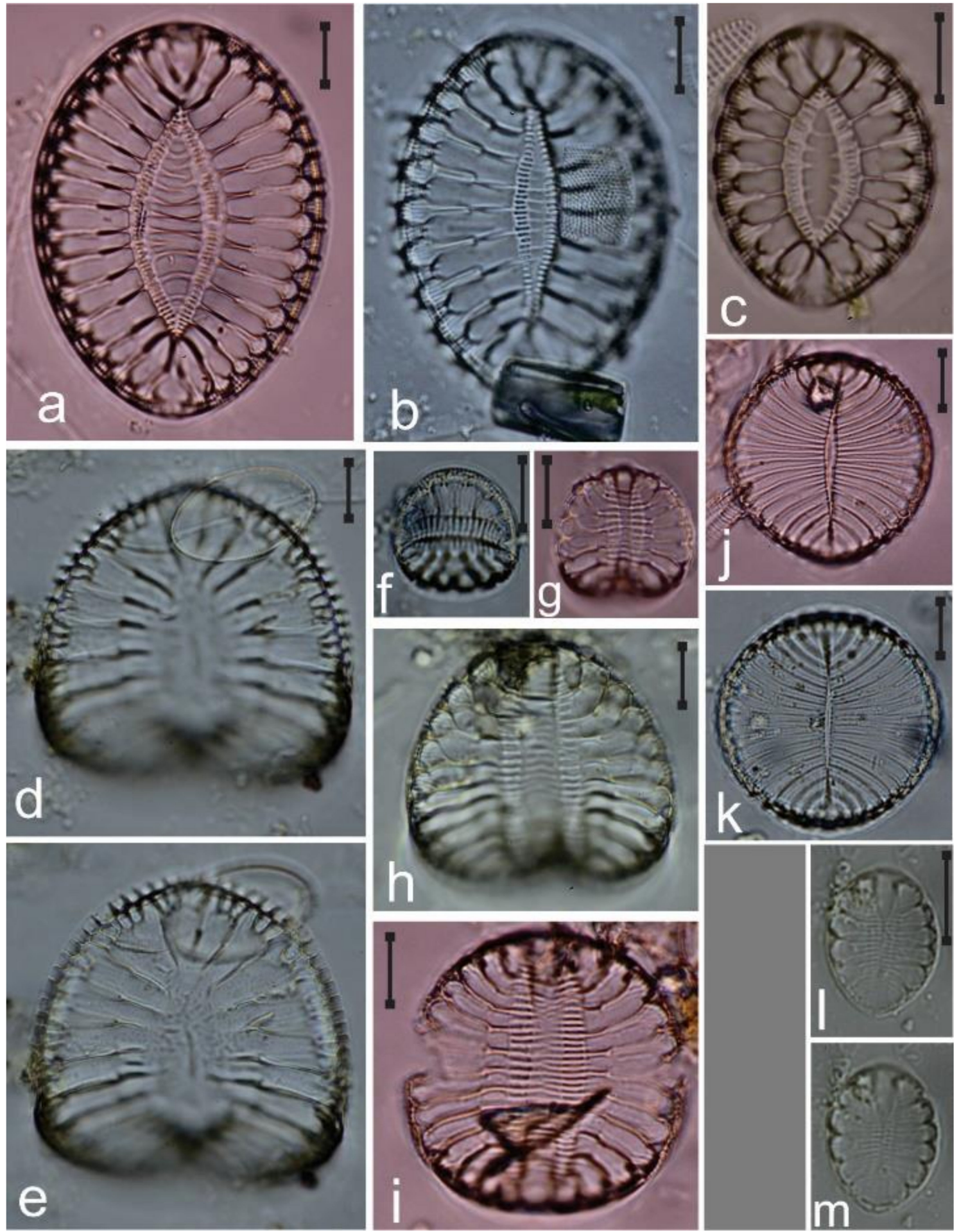

Figure 46. (a-c) Campylodiscus neofastuosus; (d,e) Coronia ambigua two focal planes; (f) Campylodiscus bicostatus; (g) C. simulans; (h,i) C. fastuosus; (j,k) C. ralfsii; $(\mathbf{l}, \mathbf{m})$ C. scalaris. Scale bar $=10 \mu \mathrm{m}$. 


\section{Discussion}

The floristic reservoir of benthic diatoms for Mexican waters continues to be surveyed with the exploration of new areas and substrata, in an effort to update the overall species list for the region [43]. This relates to the variety of substrata that benthic diatoms occupy, mainly macroalgae that harbor a notably high species richness of epiphytic diatoms that can exceed hundreds of taxa on a single host [44]. In this regard, the species richness of diatoms living on a single specimen $\left(3 \mathrm{~cm}^{2}\right)$ of Phyllodictyon pulcherrimum from the Gulf of California yielded 244 specific and infraspecific taxa, the highest number yet recorded for any Chlorophyceae host. In contrast to the observations made during the identification of the host Chlorophyceae, the allegedly dominant genus Cocconeis (Figure 2) accounted for only 12 of the total species (5\%). Thus, although the host surface may be paved and "dominated" by monogeneric species, many others may occur which show distinct fixing and growing strategies on the algal host surface. Although several of these taxa may be planktonic, such as Coscinodiscus and Chaetoceros, or Thalassiosira, they were present as epiphytes of P. pulcherrimum and are recorded with the epiphytic flora, but should be properly accounted for in numerical assessments, albeit solely when considering their relevance in a genus level analysis due to the scarceness of these forms. The eleven green algae taxa from the studies reviewed yielded a list of 300 taxa of epiphytic diatoms. The genera best represented were Mastogloia (35 species), Nitzschia (19), Navicula (19), Cocconeis (18), Diploneis (12), Licmophora (9), and Amphora (7). On P. pulcherrimum, Mastogloia and Licmophora were represented by only three and five species, respectively, while of the 300 diatom taxa recorded on these 11 Chlorophyceae taxa, only $68(27.75 \%)$ were also present on the host specimen of $P$. pulcherrimum. Of the diatom genera best represented on the eleven green algae, only 25 taxa (8.3\%) were also observed on P. pulcherrimum: Nitzschia (9), Cocconeis (7), Diploneis (6), Navicula (2), and Amphora (1). The species richness recorded from $P$. pulcherrimum surpasses all records in the 14 studies reviewed on other green macroalgae. Only [10] observed a similarly high species count with 115 diatom taxa as epiphytes on Halimeda tuna and 137 on Caulerpa taxifolia. Their findings are followed by [6], with 89 taxa on Codium spp. Other studies recorded lower numbers of epiphytic diatom taxa, such as [21] with 51 taxa on Codium latum subsp. palmeri, [18] with 63 taxa on Chaetomorpha linum, Enteromorpha intestinalis, and Ulva lactuca, and [9] with 52 taxa on Halimeda sp. Other Chlorophyceae, such as Cladophora glomerata and Cladophora sp., had only four diatom taxa that co-occurred with P. pulcherrimum (Diploneis smithii, Halamphora coffeaeformis, Nitzschia gracilis and N. linearis), but these are freshwater and estuarine forms.

In the case of the few fresh-water forms recorded, it has to be considered that the annual pp for the study area is around $100 \mathrm{~mm}$, mainly for short periods during tropical storms [45]. The runoff is mostly sporadic, characteristic of the warm months, including July, maybe related to the San José de Magdalena oasis located around $25 \mathrm{~km}$ SW. In any case, the presence of said diatom forms should be assessed in terms of their permanence to determine whether these are regular residents in the marine environment or accidental inputs. The former case would lead to a review of their taxonomic status, and as with those taxa identified only as sp., further study relying on molecular and SEM would be required.

Out of the likely explanations for the high species richness of diatoms observed on P. pulcherrimum, we are inclined to hypothesize that the more complex thallus of this macroalgae, showing a flat surface, folds, and branching renders a microhabitat heterogeneity that promotes colonization by many more diatom taxa. However, in the same area, other substrata have also yielded many diatom taxa, as seen in [2] and in an ongoing study [46] in which close to 400 taxa were observed on several macroalgae species from a pristine control site. Thus, environmental conditions seem to promote high diatom species diversity in general. Further research will reveal how host macroalgal diversity and their epiphytic diatoms combine to define an overall floristic richness for the Gulf of California.

The identified taxon Margaritum terebro, with its striking morphological form, represents a new record for Mexican coasts. On the basis of previous records, [47] suggested that this taxon is restricted to tropical areas (southeast coast of Brazil, Atlantic coast of Africa, Galapagos Islands). However, 
our recording of it in the middle area of the Gulf of California extends its distribution range to the subtropics. Likewise, Glyphodesmis rhombica had been recorded in the East Indies, Indonesia (Bali) and Byron Bay Australia [48] but not for the (eastern) Pacific Ocean. Finally, the following were all recorded for the first time on the American continent: Auricula flabelliformis and A. pulchra, Campylodiscus scalaris, Coscinodiscus mesoleius, Dimeregramma fulvum, Navicula palpebralis var. angulosa, Seminavis barbarae and Plagiotropis australis, while Nitzschia fusiformis had not been recorded previously for the Pacific Ocean.

The observations to date strongly suggest that the algal host group at the category of division determines different diatom assemblages [49]. However, many macroalgae taxa still await surveying for diatom epiphytes. Future analyses of these taxa will eventually help determine the extent of the homogeneity of diatom distribution on macroalgal hosts, as well as the potential specific relations between the host taxon and the composition of its diatom species. In addition to confirming that most diatom taxa in this region are distributed as epiphytes on macroalgal hosts, the present study of the diatom taxocoenosis living on P. pulcherrimum contributed many (38) new records to the floristics of the Mexican littorals, which was, to say the least, unexpected. The species richness of diatoms living on P. pulcherrimum is among the highest recorded for all macroalgae taxa, suggesting that the thalli of these Chlorophyceae species offer conditions that promote the settlement and growth of epiphytic diatoms unlike other macroalgae. Although it has been proposed that epiphytic diatom assemblages may be characteristic of their particular macroalgal host in terms of floristics and diversity, our findings indicate that some macroalgae taxa may offer a better substratum than others. These results allow us to confirm that surveying rare macroalgae species as hosts for epiphytic diatoms continues to offer opportunities for achieving new records of diatom taxa, or even identifying new taxa in many regions of the world.

Author Contributions: The first author (F.O.L.-F.) participated in conceptualization of the research problem, implementation of methodology for formal taxonomic analysis, overall taxa identification, and writing of the manuscript. The second author (D.A.S.B.) contributed to contextualize the scientific perspective of the taxonomic problem, reviewing species identification and new taxonomic records, plus the writing and translation of the manuscript. The third author (M.d.C.A.-C.) identified the macroalgal host, noticing and confirming the scarceness of the host taxon, and the presence of the epiphytic diatom assemblage, plus reviewed the original draft. All authors have read and agreed to the published version of the manuscript.

Funding: This research received no external funding.

Acknowledgments: F.O.L.-F. thanks the support of the Programa para el Desarrollo Profesional Docente (PRODEP) and SNI-CONACYT programs. D.A.S.B. is COFAA and EDI fellow at Instituto Politécnico Nacional. We also thank Paul C. Kersey Johnson (El Colegio Michoacán, México) for the English review of this manuscript, and Gabriela Cruz-Piñon for the map image. Finally, we acknowledge the effort by two anonymous reviewers and the assigned editor to this paper.

Conflicts of Interest: The authors declare no conflict of interest.

\section{References}

1. López-Fuerte, F.O.; Siqueiros Beltrones, D.A.; Veleva, L. Diatoms (Bacillariophyta) from artificial substrates and sediments in the Caribbean Sea off Yucatan, Mexico. Phytotaxa 2019, 406, 1-54. [CrossRef]

2. López-Fuerte, F.O.; Siqueiros Beltrones, D.A.; Jakes-Cota, U.; Tripp-Valdéz, A. New records of epizoic diatoms from the skin of stone scorpionfish Scorpaena mystes in Mexican waters. Open J. Mar. Sci. 2019, 9, 98-112. [CrossRef]

3. Martínez, Y.J.; Siqueiros Beltrones, D.A.; Ardisson, P.L. Species composition of diatom assemblages growing on fiber-glass plates and bottom sediment of a subtidal beach in the southern Gulf of Mexico. CICIMAR Oceán. 2019, 34, 29-40.

4. Tiffany, M.A. Epizoic and Epiphytic Diatoms. In The Diatom World; Seckbach, J., Kociolek, J.P., Eds.; Springer: New York, NY, USA, 2011; pp. 197-209. [CrossRef]

5. Snoeijs, P. Distribution of epiphytic diatom species composition, diversity and biomass on different macroalgal hosts along seasonal and salinity gradients in the Baltic sea. Diatom Res. 1994, 9, 189-211. [CrossRef] 
6. Siqueiros Beltrones, D.A.; Hernández Almeida, O.U. Florística de diatomeas epifitas en macroalgas de un manchón subtropical. CICIMAR Oceán. 2006, 21, 11-61.

7. Lobban, C.S.; Schefter, M.; Jordan, R.W.; Arai, Y.; Sasaki, A.; Theriot, E.C.; Ashworth, M.; Ruck, E.C.; Pennesi, C. Coral-reef diatoms (Bacillariophyta) from Guam: New records and preliminary checklist, with emphasis on epiphytic species from farmer-fish territories. Micronesica 2012, 43, 237-479.

8. Siqueiros Beltrones, D.A.; Martínez, Y.J. Prospective floristics of epiphytic diatoms on rhodophyta from the southern Gulf of Mexico. CICIMAR Oceán. 2017, 32, 35-49.

9. Park, J.; Lobban, C.; Lee, K. Diatoms associated with seaweeds from Moen Island in Chuuk Lagoon, Micronesia. Phytotaxa 2018, 351, 101-140. [CrossRef]

10. Car, A.; Witkowski, A.; Dobosz, S.; Jasprica, N.; Ljubimir, S.; Zgłobicka, I. Epiphytic diatom assemblages on invasive Caulerpa taxifolia and autochthonous Halimeda tuna and Padina sp. seaweeds in the Adriatic Sea-Summer/autumn aspect. Oceanol. Hydrobiol. St. 2019, 48, 209-226. [CrossRef]

11. Lowe, R.L.; Rosen, B.H.; Kingston, J.C. A comparison of epiphytes on Bangia atropurpurea (Rhodophyta) and Cladophora glomerata (Chlorophyta) from Northern Lake Michigan. J. Great Lakes Res. 1982, 8, 164-168. [CrossRef]

12. Stevenson, R.J.; Stoermer, E.F. Abundance patterns of diatoms on Cladophora in Lake Huron with respect to a point source of wastewater treatment plant effluent. J. Great Lakes Res. 1982, 8, 184-195. [CrossRef]

13. Dodds, W.K. Community interactions between the filamentous alga Cladophora glomerata (L.) Kuetzing, its epiphytes, and epiphyte grazers. Oecologia 1991, 85, 572-580. [CrossRef] [PubMed]

14. Hardwick, G.G.; Blinn, D.W.; Usher, H.D. Epiphytic Diatoms on Cladophora glomerata in the Colorado River, Arizona: Longitudinal and Vertical Distribution in a Regulated River. Southwest Nat. 1992, 37, 148-156. [CrossRef]

15. Car, A.; Witkowski, A.; Dobosz, S.; Burfeind, D.D.; Meinesz, A.; Jasprica, N.; Ruppel, M.; Kurzydłowski, K.J.; Płociński, T. Description of a new marine diatom, Cocconeis caulerpacola sp. nov. (Bacillariophyceae), epiphytic on invasive Caulerpa species. Eur. J. Phycol. 2012, 47, 433-448. [CrossRef]

16. El-Din, N.G.; Shaltout, N.A.; Nassar, M.Z.; Soliman, A. Ecological studies of epiphytic microalgae and epiphytic zooplankton on seaweeds of the Eastern Harbor, Alexandria, Egypt. Am. J. Environ. Sci. 2015, 11, 450-473. [CrossRef]

17. Da Silva, M.; Pereira, S.; Silva-Cunha, M.; Arruda, P.; Eskinazi-Leça, E. Community structure of epiphytic diatoms on seaweeds in Northeastern Brazil. Bot. Mar. 2016, 59, 231-240. [CrossRef]

18. Al-Harbi, S.M. Epiphytic microalgal species composition and dynamics on host green seaweeds (Ulvaphyceae) on the Northern Coast of Jeddah, Saudi Arabia. Environ. Ecol. Res. 2017, 5, 212-219. [CrossRef]

19. Ding, L.; Dai, Y.; Huang, B.; Li, Y.; Liu, R.; Shaoyang, D. Marine green alga Cladophora aokii Yamada and its epiphytes from Yinggehai, Hainan, China: Morphological taxonomy, formation, and analysis of its environmental adaption. Acta Oceanol. Sin. 2018, 37, 40-45. [CrossRef]

20. Donadel, L.; Torgan, L.; Al-Handal, A. Additional morphological features of the epiphytic diatom Cocconeis sawensis Al-Handal \& Riaux-Gobin (Cocconeidaceae, Bacillariophyta) from a coastal lagoon, Southern Brazil. Phytotaxa 2018, 371, 217-229. [CrossRef]

21. López-Fuerte, F.O.; Siqueiros Beltrones, D.A.; Yabur, R. First record of benthic diatoms (Bacillariophyceae and Fragilariophyceae) from Isla Guadalupe, Baja California, Mexico. Rev. Mex. Biodivers. 2015, 86, 281-292. [CrossRef]

22. Siqueiros Beltrones, D.A.; Martínez, Y.J.; Aldana Moreno, A. Florística exploratoria de diatomeas epifitas en Rhodophyta de Islas Revillagigedo. Cymbella 2019, 5, 98-123.

23. Siqueiros Beltrones, D.A.; Argumedo Hernández, U. Quasi monospecific proliferation of Pteroncola inane (Giffen) Round (Fragilariales; Bacillariophyceae) on blades of Eisenia arborea Areschoug. CICIMAR Oceán. 2014, 29, 57-62.

24. Leliaert, F.; Wysor, B.; Verbruggen, H.; Vlaeminck, C.; De Clerck, O. Phyllodictyon robustum (Setchell et Gardner) comb. nov. (Siphonocladales, Chlorophyta), a morphologically variable species from the tropical Pacific coast of America. Cryptogam. Algol. 2008, 29, 217-233.

25. Norris, J.N. Marine Algae of the Northern Gulf of California: Chlorophyta and Phaeophyceae. Smithson. Contr. Bot. 2010, 1-276. [CrossRef] 
26. Round, F.E.; Crawford, R.M.; Mann, D.G. The Diatoms: Biology E Morphology of the Genera; Cambridge University Press: Cambridge, UK, 1990; p. 747.

27. Guiry, M.D.; Guiry, G.M. Algaebase. Available online: http://www.algaebase.org (accessed on 23 June 2020).

28. WoRMS Editorial Board. World Register of Marine Species. Available online: http://www.marinespecies.org (accessed on 23 June 2020).

29. Schmidt, A.W.F.; Schmitz, M.; Fricke, F.; Müller, O.; Heiden, H.; Hustedt, F. Atlas der Diatomaceen-Kunde; 4 Bands, pls. 1-480; Koeltz Scientific Books: Konigstein, Germany, 1972; pp. 1874-1959.

30. Samanta, B.; Kaczmarska, I.; Ehrman, J.R. Auxosporulation in Biddulphia tridens (Ehrenberg) Ehrenberg (Mediophyceae, Bacillariophyta). Eur. J. Phycol. 2020, 55, 1-14. [CrossRef]

31. Sar, E.A. Ultrastructure of Odontella rostrata (Hustedt)Simonsen, a new record for the South Atlantic Ocean. Bot. Mar. 1989, 32, 187-189. [CrossRef]

32. Kooistra, W.H.C.F.; Forlani, G.; Sterrenburg, F.A.S.; De Stefano, M. Molecular phylogeny and morphology of the marine diatom Talaroneis posidoniae gen. et $\mathrm{sp}$. nov. (Bacillariophyta) advocate the return of the Plagiogrammaceae to the pennate diatoms. Phycologia 2004, 43, 58-67. [CrossRef]

33. Li, C.L.; Ashworth, M.P.; Witkowski, A.; Dabek, P.; Medlin, L.K.; Kooistra, W.H.C.F.; Sato, S.; Zgłobicka, I.; Kurzydłowski, K.J.; Theriot, E.C.; et al. New insights into Plagiogrammaceae (Bacillariophyta) based on multigene phylogenies and morphological characteristics with the description of a new genus and three new species. PLoS ONE 2015, 10, e0139300. [CrossRef]

34. Navarro, J.N. Marine diatoms associated with mangrove prop roots in the Indian River, Florida, USA. Bibl. Phycol. 1982, 61, 1-151.

35. López-Fuerte, F.O.; Siqueiros Beltrones, D.A.; Navarro, J.N. Benthic Diatoms Associated with Mangrove Environments in the Northwest Region of Mexico; CONABIO-UABCS-IPN: La Paz, Mexico, 2010; p. 217.

36. Stepanek, J.G.; Kociolek, J.P. Re-examination of Mereschkowsky's genus Tetramphora (Bacillariophyta) and its separation from Amphora. Diat. Res. 2016, 31, 2-123. [CrossRef]

37. Peragallo, H.; Peragallo, M. Diatomées Marines de France et des Districts Maritimes Voisins; Tempère, J., Ed.; Micrographe-Editeur: Grez-sur-Loing, France, 1897-1908; pp. 1-491.

38. Hustedt, F. Marine Littoral Diatoms of Beaufort, North Carolina; Duke University Press: Durham, NC, USA, $1955 ;$ p. 67.

39. Sterrenburg, F.A.S. Studies on the genera Gyrosigma and Pleurosigma (Bacillariophyceae). Gyrosigma reversum (Gregory) Hendey and G. naja (Meister) Sterrenburg, nov. comb. Proc. Acad. Nat. Sci. Phila. 2000, 150, 301-304.

40. Sar, E.A.; Sterrenburg, F.A.S.; Lavigne, A.S.; Sunesen, I. Diatomeas de ambientes marinos costeros de Argentina: Especies del género Pleurosigma (Pleurosigmataceae). Bol. Soc. Argent. Bot. 2013, 48, 17-51.

41. Kociolek, J.P. A checklist and preliminary bibliography of the Recent, freshwater diatoms of inland environments of the continental United States. Proc. Calif. Acad. Sci. 2005, 56, 395-525.

42. Al-Handal, A.Y.; Thomas, E.W.; Pennesi, C. Marine benthic diatoms in the newly discovered coral reefs, off Basra coast, Southern Iraq. Phytotaxa 2018, 372, 111-152. [CrossRef]

43. López-Fuerte, F.O.; Siqueiros Beltrones, D.A. A checklist of marine benthic diatoms (Bacillariophyta) from Mexico. Phytotaxa 2016, 283, 201-258. [CrossRef]

44. Siqueiros Beltrones, D.A.; Argumedo Hernández, U.; López-Fuerte, F.O. Diversity of benthic diatoms in Laguna Guerrero Negro (El Vizcaíno Biosphere Reserve), Baja California peninsula, Mexico. Rev. Mex. Biodivers. 2017, 88, 21-35. [CrossRef]

45. Rodríguez-Figueroa, G.; Shumilin, E.; Sánchez-Rodríguez, I. Heavy metal pollution monitoring using the brown seaweed Padina durvillaei in the coastal zone of the Santa Rosalía mining region, Baja California Peninsula, Mexico. J. Appl. Phycol. 2009, 21, 19-26. [CrossRef]

46. Martínez, Y.J. Respuesta de las Asociaciones de Diatomeas Bentónicas a la Presencia de Elementos Potencialmente Tóxicos. Ph.D. Thesis, Centro Interdisciplinario de Ciencia Marinas, Instituto Politécnico Nacional, La Paz, B.C.S., Mexico, 2020; pp. 1-130.

47. Souza-Mosimann, R.M.; Fernandes, L.F.; Ludwig, T.V. The estuarine benthic diatom Margaritum terebro (Bacillariophyta, Hyalodiscaceae): Morphology and taxonomy. Rev. Bras. Oceanogr. 1997, 45, 45-52. [CrossRef] 
48. Foged, N. Diatoms in Eastern Australia. Biblioth. Phycol. 1978, 41, 1-243.

49. Hernández-Almeida, O.U.; Siqueiros Beltrones, D.A. Substrate dependent differences in the structure of epiphytic vs. epilithic diatom assemblages from the southwestern coast of the Gulf of California. Bot. Mar. 2012, 55, 149-159. [CrossRef]

(C) 2020 by the authors. Licensee MDPI, Basel, Switzerland. This article is an open access article distributed under the terms and conditions of the Creative Commons Attribution (CC BY) license (http://creativecommons.org/licenses/by/4.0/). 